\title{
Chiral thiols: the assignment of their absolute configuration by ${ }^{1} \mathrm{H}-\mathrm{NMR}$
}

Silvia Porto, ${ }^{\mathrm{a}}$ José Manuel Seco, ${ }^{\mathrm{a}}$ Aurelio Ortiz, ${ }^{\mathrm{b}}$ Emilio Quiñoáa ${ }^{\mathrm{a}}$ and Ricardo Riguera*a

${ }^{a}$ Departamento de Química Orgánica, Facultad de Química y Unidad de RMN de Biomoléculas Asociada al CSIC, Universidad de Santiago de Compostela, E-15782 Santiago de Compostela, Spain

Tel/Fax: (34) 981591091; E-mail: ricardo@usc.es

${ }^{b}$ Centro de Investigación, Facultad de Ciencias Químicas, Benemérita Universidad Autónoma de Puebla, 72570 Puebla, México; E-mail: jaortiz@siu.buap.mx; Fax: 22295584; Tel: 22-295500 Ext 7518

\section{Supporting Information}




\section{Table of Contents}

\section{Experimental Section}

Chiral thiols.

General thioesterification procedure.

Synthesis of aryl-tert-butoxyacetic acids.

Scheme 1. Synthesis of the enantiomers of 16, 17, 18 .

Preparation of methyl 2-hydroxy-2-(2-naphthyl)acetates.

Preparation of methyl 2-tert-butoxy-2-(2-naphthyl)acetates.

Preparation of 2-tert-butoxy-2-(2-naphthyl)acetic acids.

NMR spectroscopy.

\section{References.}

\section{Spectroscopic data:}

$(R)$-Methyl 2-tert-butoxy-2-phenylacetate [(R)-16a].

$(S)$-Methyl 2-tert-butoxy-2-phenylacetate [(S)-16a].

$(R)$-Methyl 2-tert-butoxy-2-(2-naphthyl)acetate [ $(R)-17 \mathrm{a}]$.

$(S)$-Methyl 2-tert-butoxy-2-(2-naphthyl)acetate [ $R)-17 \mathrm{a}]$.

$(R)$-Ethyl 2-tert-butoxy-2-(9-anthryl)acetate [ $(R)-18 \mathrm{a}]$.

$(R)$-2-tert-butoxy-2-phenylacetic acid $[(R)-16]$.

$(S)$-2-tert-butoxy-2-phenylacetic acid [(S)-16].

$(R)$-2-tert-butoxy-2-(2-naphthyl)acetic acid [(R)-17].

$(S)$-2-tert-butoxy-2-(2-naphthyl)acetic acid [(S)-17].

$(R)$-2-tert-butoxy-2-(9-anthryl) acetic acid [ $(R)-18]$.

$(R)$-MPA thioester of $(S)$-butane-2-thiol [ $(R)$-MPA-5].

$(S)$-MPA thioester of $(S)$-butane-2-thiol [ $(S)$-MPA-5].

$(R)$-MPA thioester of $(S)$-octane-2-thiol [ $(R)$-MPA-6].

$(S)$-MPA thioester of $(S)$-octane-2-thiol [(S)-MPA-6].

$(R)$-MPA thioester of $(1 S, 2 S, 5 R)$-2-isopropyl-5-methylcyclohexanethiol [(R)-MPA-7].

$(S)$-MPA thioester of $(1 S, 2 S, 5 R)$-2-isopropyl-5-methylcyclohexanethiol [ $(S)$-MPA-7].

$(R)$-MPA thioester of $(R)$-ethyl 2-mercaptopropanoate [ $(R)$-MPA-8].

$(S)$-MPA thioester of $(R)$-ethyl 2-mercaptopropanoate [(S)-MPA-8].

$(R)$-MPA thioester of $(R)$-methyl 2-mercapto-2-phenylacetate [ $(R)$-MPA-9].

$(S)$-MPA thioester of $(R)$-methyl 2-mercapto-2-phenylacetate [ $(S)$-MPA-9].

$(R)$-MPA thioester of 1-thio- $\beta$-D-glucose tetraacetate [ $(R)$-MPA-10].

$(S)$-MPA thioester of 1-thio- $\beta$-D-glucose tetraacetate [ $(S)$-MPA-10].

$(R)$-MPA thioester of (3' $R, 4 S)$-3-(1'-oxobutyl-3'-mercapto)-4-isopropyl-5,5-dimethyl-

1,3-oxazolidinone [(R)-MPA-11]. 
(S)-MPA thioester of 3'R,4S)-3-(1'-oxobutyl-3'-mercapto)-4-isopropyl-5,5-dimethyl1,3-oxazolidinone [(S)-MPA-11].

( $R$ )-MPA thioester of (2' $S, 3 ' R, 4 S)$-3-(1'-oxobutyl-2-methyl-3'-mercapto)-4-isopropyl-

5,5-dimethyl-1,3-oxazolidinone [(R)-MPA-12].

$(S)$-MPA thioester of 2'S,3' $R, 4 S)$-3-(1'-oxobutyl-2-methyl-3'-mercapto)-4-isopropyl5,5-dimethyl-1,3-oxazolidinone [(S)-MPA-12].

$(R)$-MPA thioester of (3' $S, 4 S)$-3-(1'-oxobutyl-3'-mercapto-3'-phenyl)-4-isopropyl-5,5dimethyl-1,3-oxazolidinone [(R)-MPA-13].

$(S)$-MPA thioester of (3' $S, 4 S)$-3-(1'-oxobutyl-3'-mercapto-3'-phenyl)-4-isopropyl-5,5dimethyl-1,3-oxazolidinone [ $(S)$-MPA-13].

$(R)$-MPA thioester of 3-(3-oxo-7 $\alpha$-thio-17 $\beta$-hydroxy-4-androsten-17 $\alpha$-yl)propionic acid $\gamma$-lactone [(R)-MPA-14].

(S)-MPA thioester of 3-(3-oxo-7 $\alpha$-thio-17 $\beta$-hydroxy-4-androsten-17 $\alpha$-yl)propionic acid $\gamma$-lactone [(S)-MPA-14].

$(R)$-MPA thioester of thiocholesterol $[(R)-\mathrm{MPA}-15]$.

$(S)$-MPA thioester of thiocholesterol [(S)-MPA-15].

$(R)$-PTBA thioester of $(S)$-butane-2-thiol [(R)-PTBA-5].

$(S)$-PTBA thioester of $(S)$-butane-2-thiol [ $(S)$-PTBA-5].

$(R)$-2-NTBA thioester of $(S)$-butane-2-thiol [ $(R)$-2-NTBA-5].

$(S)$-2-NTBA thioester of $(S)$-butane-2-thiol [ $(S)$-2-NTBA-5].

$(R)$-2-NTBA thioester of $(S)$-octane-2-thiol [ $R$-2-NTBA-6].

$(S)$-2-NTBA thioester of $(S)$-octane-2-thiol [(S)-2-NTBA-6].

$(R)$-2-NTBA thioester of $(1 S, 2 S, 5 R)$-2-isopropyl-5-methylcyclohexanethiol $[(R)-2-$ NTBA-7].

$(S)$-2-NTBA thioester of $(1 S, 2 S, 5 R)$-2-isopropyl-5-methylcyclohexanethiol [(S)-2NTBA-7].

$(R)$-2-NTBA thioester of $(R)$-ethyl 2-mercaptopropanoate [ $(R)$-2-NTBA-8].

$(S)$-2-NTBA thioester of $(R)$-ethyl 2-mercaptopropanoate [ $(S)$-2-NTBA-8].

$(R)$-2-NTBA thioester of $(R)$-methyl 2-mercapto-2-phenylacetate [ $(R)-2-\mathrm{NTBA}-9]$.

$(S)$-2-NTBA thioester of $(R)$-methyl 2-mercapto-2-phenylacetate [ $(S)$-2-NTBA-9].

$(R)$-2-NTBA thioester of 1 -thio- $\beta$-D-glucose tetraacetate [ $(R)-2-\mathrm{NTBA}-10]$.

$(S)$-2-NTBA thioester of 1-thio- $\beta$-D-glucose tetraacetate [( $S)$-2-NTBA-10].

$(R)$-2-NTBA thioester of 3-(3-oxo-7 $\alpha$-thio-17 $\beta$-hydroxy-4-androsten-17 $\alpha$-yl)propionic acid $\gamma$-lactone [(R)-2-NTBA-14].

(S)-2-NTBA thioester of 3-(3-oxo-7 $\alpha$-thio-17 $\beta$-hydroxy-4-androsten-17 $\alpha$-yl)propionic acid $\gamma$-lactone [(S)-2-NTBA-14].

$(R)$-2-NTBA thioester of thiocholesterol [ $(R)-2-\mathrm{NTBA}-15]$.

$(S)$-2-NTBA thioester of thiocholesterol [(S)-2-NTBA-15].

\section{${ }^{1} \mathrm{H}$ NMR and ${ }^{13} \mathrm{C}$ NMR spectra of selected compounds:}

Figure 1S. ${ }^{1} \mathrm{H}$ NMR and ${ }^{13} \mathrm{C}$ NMR spectra of $(R)-16 \mathrm{a}$.

Figure 2S. ${ }^{1} \mathrm{H}$ NMR and ${ }^{13} \mathrm{C}$ NMR spectra of $(S)-16$ a.

Figure 3S. ${ }^{1} \mathrm{H}$ NMR and ${ }^{13} \mathrm{C}$ NMR spectra of $(R)-17$ a.

Figure 4S. ${ }^{1} \mathrm{H}$ NMR and ${ }^{13} \mathrm{C}$ NMR spectra of $(S)-17 \mathrm{a}$.

Figure 5S. ${ }^{1} \mathrm{H}$ NMR and ${ }^{13} \mathrm{C}$ NMR spectra of $(R)-18$ a.

Figure 6S. ${ }^{1} \mathrm{H}$ NMR and ${ }^{13} \mathrm{C}$ NMR spectra of $(R)-16$.

Figure 7S. ${ }^{1} \mathrm{H}$ NMR and ${ }^{13} \mathrm{C}$ NMR spectra of $(S)-16$.

Figure 8S. ${ }^{1} \mathrm{H}$ NMR and ${ }^{13} \mathrm{C}$ NMR spectra of $(R)-17$.

Figure 9S. ${ }^{1} \mathrm{H}$ NMR and ${ }^{13} \mathrm{C}$ NMR spectra of $(S)-17$. 
Figure 10S. ${ }^{1} \mathrm{H}$ NMR and ${ }^{13} \mathrm{C}$ NMR spectra of $(R)-18$.

Figure 11S. ${ }^{1} \mathrm{H}$ NMR and ${ }^{13} \mathrm{C}$ NMR spectra of $(R)$-MPA-5.

Figure 12S. ${ }^{1} \mathrm{H}$ NMR and ${ }^{13} \mathrm{C}$ NMR spectra of $(S)$-MPA-5.

Figure 13S. ${ }^{1} \mathrm{H}$ NMR and ${ }^{13} \mathrm{C}$ NMR spectra of $(R)$-MPA-6.

Figure 14S. ${ }^{1} \mathrm{H}$ NMR and ${ }^{13} \mathrm{C}$ NMR spectra of $(S)$-MPA-6.

Figure 15S. ${ }^{1} \mathrm{H}$ NMR and ${ }^{13} \mathrm{C}$ NMR spectra of $(R)$-MPA-7.

Figure 16S. ${ }^{1} \mathrm{H}$ NMR and ${ }^{13} \mathrm{C}$ NMR spectra of $(S)$-MPA-7.

Figure 17S. ${ }^{1} \mathrm{H}$ NMR and ${ }^{13} \mathrm{C}$ NMR spectra of $(R)$-MPA-8.

Figure 18S. ${ }^{1} \mathrm{H}$ NMR and ${ }^{13} \mathrm{C}$ NMR spectra of $(S)$-MPA-8.

Figure 19S. ${ }^{1} \mathrm{H}$ NMR and ${ }^{13} \mathrm{C}$ NMR spectra of $(R)$-MPA-9.

Figure 20S. ${ }^{1} \mathrm{H}$ NMR and ${ }^{13} \mathrm{C}$ NMR spectra of $(S)$-MPA-9.

Figure 21S. ${ }^{1} \mathrm{H}$ NMR and ${ }^{13} \mathrm{C}$ NMR spectra of $(R)$-MPA-10.

Figure 22S. ${ }^{1} \mathrm{H}$ NMR and ${ }^{13} \mathrm{C}$ NMR spectra of $(S)$-MPA-10.

Figure 23S. ${ }^{1} \mathrm{H}$ NMR and ${ }^{13} \mathrm{C}$ NMR spectra of $(R)$-MPA- 11 .

Figure 24S. ${ }^{1} \mathrm{H}$ NMR and ${ }^{13} \mathrm{C}$ NMR spectra of $(S)$-MPA-11.

Figure 25S. ${ }^{1} \mathrm{H}$ NMR and ${ }^{13} \mathrm{C}$ NMR spectra of $(R)$-MPA-12.

Figure 26S. ${ }^{1} \mathrm{H}$ NMR and ${ }^{13} \mathrm{C}$ NMR spectra of $(S)$-MPA-12.

Figure 27S. ${ }^{1} \mathrm{H}$ NMR and ${ }^{13} \mathrm{C}$ NMR spectra of $(R)$-MPA-13.

Figure 28S. ${ }^{1} \mathrm{H}$ NMR and ${ }^{13} \mathrm{C}$ NMR spectra of $(S)$-MPA-13.

Figure 29S. ${ }^{1} \mathrm{H}$ NMR and ${ }^{13} \mathrm{C}$ NMR spectra of $(R)$-MPA-14.

Figure 30S. ${ }^{1} \mathrm{H}$ NMR and ${ }^{13} \mathrm{C}$ NMR spectra of $(S)$-MPA-14.

Figure 31S. ${ }^{1} \mathrm{H}$ NMR and ${ }^{13} \mathrm{C}$ NMR spectra of $(R)$-MPA-15.

Figure 32S. ${ }^{1} \mathrm{H}$ NMR and ${ }^{13} \mathrm{C}$ NMR spectra of $(S)$-MPA- 15.

Figure 33S. ${ }^{1} \mathrm{H}$ NMR and ${ }^{13} \mathrm{C}$ NMR spectra of $(R)$-PTBA-5.

Figure 34S. ${ }^{1} \mathrm{H}$ NMR and ${ }^{13} \mathrm{C}$ NMR spectra of $(S)$-PTBA-5.

Figure 35S. ${ }^{1} \mathrm{H}$ NMR and ${ }^{13} \mathrm{C}$ NMR spectra of $(R)-2-\mathrm{NTBA}-5$.

Figure 36S. ${ }^{1} \mathrm{H}$ NMR and ${ }^{13} \mathrm{C}$ NMR spectra of $(S)$-2-NTBA-5.

Figure 37S. ${ }^{1} \mathrm{H}$ NMR and ${ }^{13} \mathrm{C}$ NMR spectra of $(R)-2-\mathrm{NTBA}-6$.

Figure 38S. ${ }^{1} \mathrm{H}$ NMR and ${ }^{13} \mathrm{C}$ NMR spectra of $(S)$-2-NTBA-6.

Figure 39S. ${ }^{1} \mathrm{H}$ NMR and ${ }^{13} \mathrm{C}$ NMR spectra of $(R)-2-\mathrm{NTBA}-7$.

Figure 40S. ${ }^{1} \mathrm{H}$ NMR and ${ }^{13} \mathrm{C}$ NMR spectra of $(S)$-2-NTBA-7.

Figure 41S. ${ }^{1} \mathrm{H}$ NMR and ${ }^{13} \mathrm{C}$ NMR spectra of $(R)-2-N T B A-8$.

Figure 42S. ${ }^{1} \mathrm{H}$ NMR and ${ }^{13} \mathrm{C}$ NMR spectra of $(S)-2-\mathrm{NTBA}-8$.

Figure 43S. ${ }^{1} \mathrm{H}$ NMR and ${ }^{13} \mathrm{C}$ NMR spectra of $(R)-2-\mathrm{NTBA}-9$.

Figure 44S. ${ }^{1} \mathrm{H}$ NMR and ${ }^{13} \mathrm{C}$ NMR spectra of $(S)$-2-NTBA-9.

Figure 45S. ${ }^{1} \mathrm{H}$ NMR and ${ }^{13} \mathrm{C}$ NMR spectra of $(R)-2-N T B A-10$.

Figure 46S. ${ }^{1} \mathrm{H}$ NMR and ${ }^{13} \mathrm{C}$ NMR spectra of $(S)$-2-NTBA-10.

Figure 47S. ${ }^{1} \mathrm{H}$ NMR and ${ }^{13} \mathrm{C}$ NMR spectra of $(R)-2-\mathrm{NTBA}-14$.

Figure 48S. ${ }^{1} \mathrm{H}$ NMR and ${ }^{13} \mathrm{C}$ NMR spectra of $(S)$-2-NTBA-14.

Figure 49S. ${ }^{1} \mathrm{H}$ NMR and ${ }^{13} \mathrm{C}$ NMR spectra of $(R)-2-N T B A-15$.

Figure 50S. ${ }^{1} \mathrm{H}$ NMR and ${ }^{13} \mathrm{C}$ NMR spectra of $(S)-2-N T B A-15$.

Crystal Structure of $(S)$-MPA thioester of 1-thio- $\beta$-D-glucose tetraacetate $[(S)$ MPA-10].

Figure 51S. Diffraction X-ray spectroscopy of the $(S)$-MPA thioester of 1 -thio- $\beta$-Dglucose tetraacetate $[(S)-\mathrm{MPA}-10]$. 


\section{Experimental Section}

\section{Chiral thiols.}

Chiral thiols (R)-methyl 2-mercapto-2-phenylacetate (9), 1-thio- $\beta$-D-glucose tetraacetate (10) and thiocholesterol (15) are commercially available.

Chiral thiols 5, 6, 7 and $\mathbf{8}$ were obtained from the corresponding commercially available chiral secondary alcohols according to reference 1.

Chiral thiols 11, 12 y 13 were obtained according to reference 2.

Chiral thiols 14 was obtained from spironolactone according to reference 3.

\section{General Thioesterification Procedure.}

The MPA, PTBA and 2-NTBA thioesters were prepared by treatment of the thiol $(1.0$ equivalent) with the corresponding $(R)$ - and $(S)$-MPA, $(R)$ - and $(S)$-PTBA or $(R)$ - and (S)-2-NTBA (1.2 equivalents) in the presence of 1-(3-dimethylaminopropyl)-3ethylcarbodiimide hydrochloride (EDCHCl, 1.2 equivalents) and DMAP (1.0 equivalents) in dry $\mathrm{CH}_{2} \mathrm{Cl}_{2}$ and under a nitrogen atmosphere. The reaction mixtures were stirred at r.t. for 2-4 h approximately (tlc monitoring). Next, the organic layers were sequentially washed with water, $\mathrm{HCl}(1 \mathrm{~N})$, water, $\mathrm{NaHCO}_{3}$ (sat) and water. Then, the layers were dried (anh. $\mathrm{NaSO}_{4}$ ) and concentrated under reduced pressure to provide the corresponding thioesters. If needed, further purification was achieved by means of flash column chromatography (silica gel 230-400; elution with hexane/ethyl acetate mixtures).

To prepare the thioesters of 1-thio- $\beta$-D-glucose tetraacetate (14), MPA or 2-NTBA and $\mathrm{EDCHCl}$ were previously mixed up in dry $\mathrm{CH}_{2} \mathrm{Cl}_{2}$. The mixture was stirred for $1 \mathrm{~h}$ and then, the thiol and DMAP were added.

\section{Synthesis of aryl-tert-butoxyacetic acids.}

$(R)$ - y $(S)$-PTBA $(\mathbf{1 6})$ were prepared from $(R)$ - y $(S)$-mandelic acid, $(R)$ - and $(S)$-2NTBA (17) from $(R)$ - and (S)-2-hydroxy-2-(2-naphthyl)acetic acid and ( $R$ )-9-ATBA (18) from ethyl (R)-2-hydroxy-2-(9-anthryl)acetate as shown in Scheme 1 (reference 4). Detailed procedure follows for the synthesis of 2-NTBA (17). Analogous procedures were used for PTBA (16) and 9-ATBA (18). 


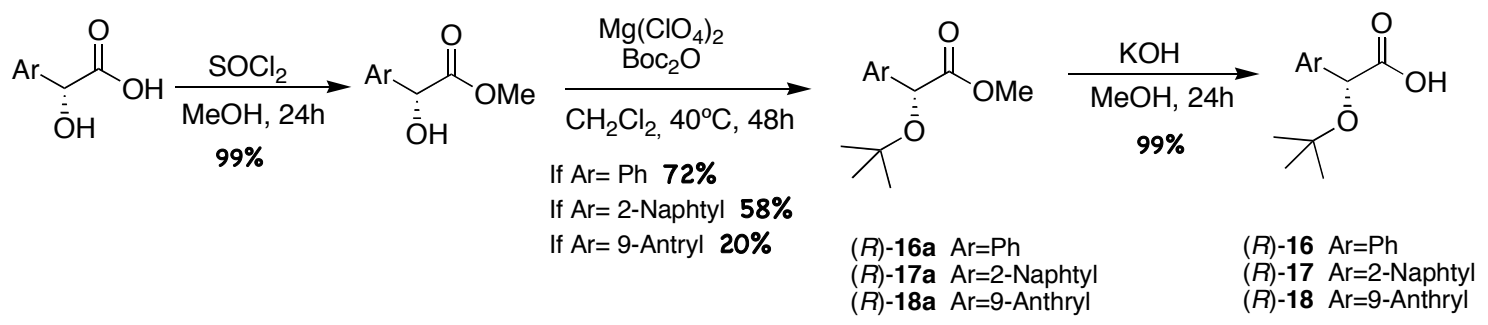

Scheme 1. Synthesis of $(R)$ - and $(S)$-PTBA $(\mathbf{1 6}),(R)$ - and $(S)$-2-NTBA $(17)$ and $(R)-9$ ATBA (18).

Preparation of methyl 2-hydroxy-2-(2-naphthyl)acetates. Thionyl chloride (196 $\mu \mathrm{L}$, $2.7 \mathrm{mmol})$ was added dropwise to a solution of $(R)$ - or $(S)$-2-hydroxy-2-(2naphthyl)acetic acid (500 mg, $2.5 \mathrm{mmol})$ in $25 \mathrm{~mL}$ of dry $\mathrm{MeOH}$ at $0^{\circ} \mathrm{C}$. The reaction was stirred at $\mathrm{rt}$ for $24 \mathrm{~h}$. Next, the $\mathrm{MeOH}$ was evaporated under reduce pressure to yield $(R)$ - or (S)-methyl 2-hydroxy-2-(2-naphthyl)acetate (99\% yield).

Preparation of methyl 2-tert-butoxy-2-(2-naphthyl)acetates. $(R)$ - or $(S)$-methyl 2hydroxy-2-(2-naphthyl)acetate $(535 \mathrm{mg}, 2.5 \mathrm{mmol})$ and $\mathrm{Mg}\left(\mathrm{ClO}_{4}\right)_{2}(56 \mathrm{mg}, 0.25 \mathrm{mmol}$, anhyd) were dissolved in dry $\mathrm{CH}_{2} \mathrm{Cl}_{2} \quad(3 \mathrm{~mL})$. Then, $\mathrm{Boc}_{2} \mathrm{O} \quad(1250$ $\mathrm{mg}, 5.7 \mathrm{mmol}$ ) was added and the resulting mixture was heated at a $40^{\circ} \mathrm{C}$ for $48 \mathrm{~h}$. Next, the mixture was diluted with water $(10 \mathrm{~mL})$ and extracted with $\mathrm{CH}_{2} \mathrm{Cl}_{2}(10 \mathrm{~mL})$. The organic layer was separated and dried with $\mathrm{Na}_{2} \mathrm{SO}_{4}$ (anhyd). After filtration, it was evaporated under reduce pressure. Column chromatography (silica gel; hexane/ethyl acetate 9:1) yielded pure $(\boldsymbol{R})-\mathbf{1 7 a}$ or $(S)-17 \mathbf{a}(58 \%$ yield). $(R)$ - and $(S)$-methyl 2-tertbutoxy-2-phenylacetate $[(\boldsymbol{R})-\mathbf{1 6 a}$ and $(\boldsymbol{S})$-16a $]$ and (R)-ethyl 2-tert-butoxy-2-(9anthryl)acetate $[(\boldsymbol{R})-\mathbf{1 8 a}]$ were obtained in a similar way $(72 \%$ and $20 \%$ yields respectively).

Preparation of 2-tert-butoxy-2-(2-naphthyl)acetic acids. $(R)$ - or $(S)$-methyl 2-tertbutoxy-2-(2-naphthyl)acetate $(394 \mathrm{mg}, 1.4 \mathrm{mmol})$ was added to a solution of $\mathrm{KOH}$ in $\mathrm{MeOH}(10 \% ; 15 \mathrm{~mL})$ and the mixture was stirred at $\mathrm{rt}$ for $8 \mathrm{~h}$. Then, $1 \mathrm{M} \mathrm{HCl}$ was added until acidic $\mathrm{pH}$ was reached. The resulting precipitate was extracted with $\mathrm{CH}_{2} \mathrm{Cl}_{2}(3 \times 25$ $\mathrm{mL}$ ) and the combined organic layers were washed with water, dried with $\mathrm{Na}_{2} \mathrm{SO}_{4}$ (anhyd), filtered and evaporated under reduce pressure. $(R)$ - or $(S)$-2-tert-butoxy-2-(2naphthyl)acetic acid [(R)-17 y $(\boldsymbol{S})$-17] were obtained in high yields $(99 \%)$. $(R)$ - and $(S)$ 2-tert-butoxy-2-phenylacetic acid $[(\boldsymbol{R})-16$ and $(S)-16]$ and $(R)$-2-tert-butoxy-2-(9anthryl)acetate $[(\boldsymbol{R})-\mathbf{1 8}]$ were obtained in a similar way (99\% yields). 


\section{NMR spectroscopy.}

${ }^{1} \mathrm{H}$ and ${ }^{13} \mathrm{C}$ NMR spectra of samples in $\mathrm{CDCl}_{3}$ were recorded at 500, 300 and $250 \mathrm{MHz}$. ${ }^{1} \mathrm{H}$ chemical shifts (ppm) are internally referenced to the TMS signal $(0.00 \mathrm{ppm}) .{ }^{13} \mathrm{C}$ chemical shifts (ppm) are internally referenced to $\mathrm{CDCl}_{3}(77.0 \mathrm{ppm}) . J$ values are recorded in $\mathrm{Hz}$.

\section{References.}

[1] Strijtveen, B.; Kellogg, R. M. J. Org. Chem. 1986, 51, 3664-3671.

[2] Ortiz, A.; Hernández, H.; Mendoza, G.; Quintero, L.; Bernès, S. Tetrahedron Lett. 2005, 46, 2243-2246.

[3] Wallace, O. W.; Springer, D. M. Tetrahedron Lett. 1998, 39, 2693-2694.

[4] (a) Kolasa, T.; Miller, M. J.; J. Org. Chem. 1987, 52, 4978-4984. (b) Bartoli, G.; Bosco, M.; Locatelli, M.; Marcantoni, E.; Melchiorre, P.; Sambri, L. Org. Lett. 2005, 7, 427-430. 


\section{Spectroscopic data}

\section{(R)-Methyl 2-tert-butoxy-2-phenylacetate [(R)-16a]}

$[\alpha]_{\mathrm{D}}=-32.75\left(c=2.50, \mathrm{CHCl}_{3}\right) ;{ }^{1} \mathrm{H} \mathrm{NMR}\left(250.13 \mathrm{MHz}, \mathrm{CDCl}_{3}\right) \delta(\mathrm{ppm}): 1.26(\mathrm{~s}, 9 \mathrm{H})$, $3.70(\mathrm{~s}, 3 \mathrm{H}), 5.09$ (s, 1H), 7.25-7.37 (m, 3H), 7.47 (dd, $J=1.6,7.6 \mathrm{~Hz}, 2 \mathrm{H}) ;{ }^{13} \mathrm{C} \mathrm{NMR}$ $\left(62.89 \mathrm{MHz}, \mathrm{CDCl}_{3}\right) \delta(\mathrm{ppm}):$ 27.9, 52.1, 73.7, 75.7, 126.5, 127.9, 128.3, 138.5, 173.1; HRMS (ESI): $\mathrm{m} / \mathrm{z}$ calcd for $\mathrm{C}_{13} \mathrm{H}_{18} \mathrm{O}_{3} \mathrm{Na}[\mathrm{M}+\mathrm{Na}]^{+}: 245.1148$, found: 245.1150 .

\section{(S)-Methyl 2-tert-butoxy-2-phenylacetate [(S)-16a]}

$[\alpha]_{\mathrm{D}}=+15.43\left(c=3.26, \mathrm{CHCl}_{3}\right) ;{ }^{1} \mathrm{H} \mathrm{NMR}\left(250.13 \mathrm{MHz}, \mathrm{CDCl}_{3}\right) \delta(\mathrm{ppm}): 1.26(\mathrm{~s}, 9 \mathrm{H})$, $3.70(\mathrm{~s}, 3 \mathrm{H}), 5.09$ (s, 1H), 7.25-7.37 (m, 3H), 7.47 (dd, $J=1.6,7.59 \mathrm{~Hz}, 2 \mathrm{H}) ;{ }^{13} \mathrm{C} \mathrm{NMR}$ $\left(62.89 \mathrm{MHz}, \mathrm{CDCl}_{3}\right) \delta(\mathrm{ppm}): 27.9,52.2,73.7,75.8,126.5,128.0,128.4,138.5,173.2$; HRMS (ESI): $\mathrm{m} / \mathrm{z}$ calcd for $\mathrm{C}_{13} \mathrm{H}_{18} \mathrm{O}_{3} \mathrm{Na}[\mathrm{M}+\mathrm{Na}]^{+}: 245.1148$, found: 245.1156 .

(R)-Methyl 2-tert-butoxy-2-(2-naphthyl)acetate [(R)-17a]

$[\alpha]_{\mathrm{D}}=-53.57\left(c=1.40, \mathrm{CHCl}_{3}\right) ;{ }^{1} \mathrm{H}$ NMR $\left(250.13 \mathrm{MHz}, \mathrm{CDCl}_{3}\right) \delta(\mathrm{ppm}): 1.28(\mathrm{~s}, 9 \mathrm{H})$, $3.68(\mathrm{~s}, 3 \mathrm{H}), 5.26(\mathrm{~s}, 1 \mathrm{H}), 7.40-7.48(\mathrm{~m}, 2 \mathrm{H}), 7.60(\mathrm{dd}, J=1.6,8.5 \mathrm{~Hz}, 1 \mathrm{H}), 7.70-7.85$ $(\mathrm{m}, 3 \mathrm{H}), 7.95(\mathrm{~s}, 1 \mathrm{H}) ;{ }^{13} \mathrm{C} \mathrm{NMR}\left(62.89 \mathrm{MHz}, \mathrm{CDCl}_{3}\right) \delta(\mathrm{ppm}): 27.9,52.1,73.8,75.8$, 124.3, 125.5, 125.9, 126.0, 127.5, 128.0, 128.1, 132.9, 133.0, 135.9, 173.0; HRMS (EI): $\mathrm{m} / \mathrm{z}$ calcd for $\mathrm{C}_{17} \mathrm{H}_{20} \mathrm{O}_{3}\left[\mathrm{M}^{+}\right]$: 272.1412, found: 272.1406 .

\section{(S)-Methyl 2-tert-butoxy-2-(2-naphthyl)acetate [(S)-17a]}

$[\alpha]_{\mathrm{D}}=+59.65\left(c=1.16, \mathrm{CHCl}_{3}\right) ;{ }^{1} \mathrm{H}$ NMR $\left(250.13 \mathrm{MHz}, \mathrm{CDCl}_{3}\right) \delta(\mathrm{ppm}): 1.28(\mathrm{~s}, 9 \mathrm{H})$, $3.68(\mathrm{~s}, 3 \mathrm{H}), 5.26(\mathrm{~s}, 1 \mathrm{H}), 7.40-7.49(\mathrm{~m}, 2 \mathrm{H}), 7.60(\mathrm{dd}, J=1.6,8.5 \mathrm{~Hz}, 1 \mathrm{H}), 7.77-7.85$ $(\mathrm{m}, 3 \mathrm{H}), 7.94(\mathrm{~s}, 1 \mathrm{H}) ;{ }^{13} \mathrm{C} \mathrm{NMR}\left(62.89 \mathrm{MHz}, \mathrm{CDCl}_{3}\right) \delta(\mathrm{ppm}): 27.9$, 52.1, 73.8, 75.8, $124.4,125.5,126.0,127.5,128.0,128.1,133.0,133.1,136.0,173.0 ; \mathrm{HRMS}(\mathrm{EI}): \mathrm{m} / \mathrm{z}$ calcd for $\mathrm{C}_{17} \mathrm{H}_{20} \mathrm{O}_{3}\left[\mathrm{M}^{+}\right]$: 272.1412, found: 272.1409 .

\section{(R)-Ethyl 2-tert-butoxy-2-(9-anthryl)acetate [(R)-18a]}

$[\alpha]_{\mathrm{D}}=-107.16\left(c=1.76, \mathrm{CHCl}_{3}\right) ;{ }^{1} \mathrm{H} \mathrm{RMN}\left(250.13 \mathrm{MHz}, \mathrm{CDCl}_{3}\right) \delta(\mathrm{ppm}): 0.98(\mathrm{t}, J=$ $7.1 \mathrm{~Hz}, 3 \mathrm{H}), 1.21(\mathrm{~s}, 9 \mathrm{H}), 3.91-4.15(\mathrm{~m}, 2 \mathrm{H}), 6.49$ (s, 1H), 7.39-7.45 (m, 2H), 7.49-7.55 $(\mathrm{m}, 2 \mathrm{H}), 7.95(\mathrm{~d}, J=8.4 \mathrm{~Hz}, 2 \mathrm{H}), 8.38(\mathrm{~s}, 1 \mathrm{H}), 8.68(\mathrm{~d}, J=5.3 \mathrm{~Hz}, 2 \mathrm{H}) ;{ }^{13} \mathrm{C} \mathrm{NMR}$ $\left(62.89 \mathrm{MHz}, \mathrm{CDCl}_{3}\right) \delta(\mathrm{ppm}): 13.9,28.1,60.9,69.4,76.0,124.7,125.8,127.1,128.4$, 
128.9, 129.8, 131.1, 131.6, 134.0, 172.8; HRMS (EI): m/z calcd for $\mathrm{C}_{22} \mathrm{H}_{24} \mathrm{O}_{3}\left[\mathrm{M}^{+}\right]$: 336.1725, found: 336.1722 .

\section{$(R)$-2-tert-butoxy-2-phenylacetic acid [(R)-16]}

$[\alpha]_{\mathrm{D}}=-111.80\left(c=0.55, \mathrm{CHCl}_{3}\right) ;{ }^{1} \mathrm{H} \mathrm{NMR}\left(250.13 \mathrm{MHz}, \mathrm{CDCl}_{3}\right) \delta(\mathrm{ppm}): 1.16(\mathrm{~s}, 9 \mathrm{H})$, $4.99(\mathrm{~s}, 1 \mathrm{H}), 7.15-7.27(\mathrm{~m}, 3 \mathrm{H}), 7.38(\mathrm{~d}, J=6.1 \mathrm{~Hz}, 2 \mathrm{H}), 10.7$ (br s, $1 \mathrm{H}) ;{ }^{13} \mathrm{C} \mathrm{NMR}$ $\left(62.89 \mathrm{MHz}, \mathrm{CDCl}_{3}\right) \delta(\mathrm{ppm}): 27.9,73.1,76.6,126.5,128.2,128.4,137.7,176.1$; HRMS (ESI): $\mathrm{m} / \mathrm{z}$ calcd for $\mathrm{C}_{12} \mathrm{H}_{16} \mathrm{O}_{3} \mathrm{Na}[\mathrm{M}+\mathrm{Na}]^{+}: 231.0992$, found: 231.0997 .

(S)-2-tert-butoxy-2-phenylacetic acid [(S)-16]

$[\alpha]_{\mathrm{D}}=+110.96\left(c=0.57, \mathrm{CHCl}_{3}\right) ;{ }^{1} \mathrm{H} \mathrm{NMR}\left(250.13 \mathrm{MHz}, \mathrm{CDCl}_{3}\right) \delta(\mathrm{ppm}): 1.16(\mathrm{~s}, 9 \mathrm{H})$, $4.99(\mathrm{~s}, 1 \mathrm{H}), 7.16-7.28(\mathrm{~m}, 3 \mathrm{H}), 7.38(\mathrm{~d}, J=6.1 \mathrm{~Hz}, 2 \mathrm{H}), 10.7$ (br s, $1 \mathrm{H}) ;{ }^{13} \mathrm{C} \mathrm{NMR}$ $\left(62.89 \mathrm{MHz}, \mathrm{CDCl}_{3}\right) \delta(\mathrm{ppm}):$ 27.9, 73.2, 76.7, 126.5, 128.2, 128.4, 137.7, 175.9; HRMS (ESI): $\mathrm{m} / \mathrm{z}$ calcd for $\mathrm{C}_{12} \mathrm{H}_{16} \mathrm{O}_{3} \mathrm{Na}[\mathrm{M}+\mathrm{Na}]^{+}:$231.0992, found: 231.0995 .

(R)-2-tert-butoxy-2-(2-naphthyl)acetic acid [(R)-17]

$[\alpha]_{\mathrm{D}}=-102.60\left(c=1.00, \mathrm{CHCl}_{3}\right) ;{ }^{1} \mathrm{H} \mathrm{NMR}\left(250.13 \mathrm{MHz}, \mathrm{CDCl}_{3}\right) \delta(\mathrm{ppm}): 1.30(\mathrm{~s}, 9 \mathrm{H})$, 5.24 (s, 1H), $7.47(\mathrm{dd}, J=3.3,6.2, \mathrm{~Hz}, 2 \mathrm{H}), 7.57$ (dd, $J=1.4,8.6 \mathrm{~Hz}, 1 \mathrm{H}), 7.78-7.86$ (m, $3 \mathrm{H}), 7.93(\mathrm{~s}, 1 \mathrm{H}) ;{ }^{13} \mathrm{C} \mathrm{NMR}\left(62.89 \mathrm{MHz}, \mathrm{CDCl}_{3}\right) \delta(\mathrm{ppm}): 28.1,73.4,77.4,124.1$, 125.8, 126.3, 127.7, 128.1, 128.4, 133.0, 133.2, 135.1, 173.9; HRMS (EI): m/z calcd for $\mathrm{C}_{16} \mathrm{H}_{18} \mathrm{O}_{3}\left[\mathrm{M}^{+}\right]: 258.1256$, found: 258.1247 .

\section{(S)-2-tert-butoxy-2-(2-naphthyl)acetic acid [(S)-17]}

$[\alpha]_{\mathrm{D}}=+100.00\left(c=1.55, \mathrm{CHCl}_{3}\right) ;{ }^{1} \mathrm{H}$ NMR $\left(250.13 \mathrm{MHz}, \mathrm{CDCl}_{3}\right) \delta(\mathrm{ppm}): 1.29(\mathrm{~s}, 9 \mathrm{H})$, $5.24(\mathrm{~s}, 1 \mathrm{H}), 7.44-7.50(\mathrm{~m}, 2 \mathrm{H}), 7.57(\mathrm{dd}, J=1.7,8.6 \mathrm{~Hz}, 1 \mathrm{H}), 7.78-7.85(\mathrm{~m}, 3 \mathrm{H}), 7.93$ $(\mathrm{s}, 1 \mathrm{H}) ;{ }^{13} \mathrm{C} \mathrm{NMR}\left(62.89 \mathrm{MHz}, \mathrm{CDCl}_{3}\right) \delta(\mathrm{ppm}): 28.0,73.4,77.3,124.1,125.8,126.3$, 127.6, 128.1, 128.4, 133.0, 133.2, 135.1, 174.2; HRMS (EI): m/z calcd for $\mathrm{C}_{16} \mathrm{H}_{18} \mathrm{O}_{3}$ $\left[\mathrm{M}^{+}\right]: 258.1256$, found: 258.1251 .

\section{(R)-2-tert-butoxy-2-(9-anthryl)acetic acid [(R)-18]}

$[\alpha]_{\mathrm{D}}=-99.11\left(c=0.54, \mathrm{CHCl}_{3}\right) ;{ }^{1} \mathrm{H} \mathrm{NMR}\left(250.13 \mathrm{MHz}, \mathrm{CDCl}_{3}\right) \delta(\mathrm{ppm}): 1.17(\mathrm{~s}, 9 \mathrm{H})$, $6.54(\mathrm{~s}, 1 \mathrm{H}), 7.42-7.55(\mathrm{~m}, 4 \mathrm{H}), 8.00(\mathrm{~d}, J=8.3 \mathrm{~Hz}, 2 \mathrm{H}), 8.42-8.46(\mathrm{~m}, 3 \mathrm{H}) ;{ }^{13} \mathrm{C} \mathrm{NMR}$ (62.89 MHz, $\left.\mathrm{CDCl}_{3}\right) \delta(\mathrm{ppm}): 28.2,69.0,77.4,124.9,126.5,127.2,129.2,129.3,129.6$, 130.0, 131.6, 134.1, 174.6; HRMS (EI): $\mathrm{m} / \mathrm{z}$ calcd for $\mathrm{C}_{20} \mathrm{H}_{20} \mathrm{O}_{3}\left[\mathrm{M}^{+}\right]: 308.1412$, found: 308.1411 . 
(R)-MPA thioester of $(S)$-butane-2-thiol [(R)-MPA-5]

$[\alpha]_{\mathrm{D}}=+3.06\left(c=0.85, \mathrm{CHCl}_{3}\right) ;{ }^{1} \mathrm{H} \mathrm{NMR}\left(250.13 \mathrm{MHz}, \mathrm{CDCl}_{3}\right) \delta(\mathrm{ppm}): 0.88(\mathrm{t}, J=7.4$ $\mathrm{Hz}, 3 \mathrm{H}), 1.29$ (d, $J=6.9 \mathrm{~Hz}, 3 \mathrm{H}), 1.50-1.60$ (m, 2H), 3.39-3.53 (m, 1H), 3.46 (s, 3H) , $4.72(\mathrm{~s}, 1 \mathrm{H}), 7.32-7.45(\mathrm{~m}, 5 \mathrm{H}) ;{ }^{13} \mathrm{C} \mathrm{NMR}\left(62.89 \mathrm{MHz}, \mathrm{CDCl}_{3}\right) \delta(\mathrm{ppm}): 11.3,20.7$, 29.5, 40.0, 57.9, 89.0, 127.0, 128.6, 136.4, 200.4; HRMS (ESI): m/z calcd for $\mathrm{C}_{13} \mathrm{H}_{18} \mathrm{O}_{2} \mathrm{SNa}[\mathrm{M}+\mathrm{Na}]^{+}: 261.0920$, found: 261.0918 .

(S)-MPA thioester of $(S)$-butane-2-thiol [(S)-MPA-5]

$[\alpha]_{\mathrm{D}}=+32.87\left(c=0.83, \mathrm{CHCl}_{3}\right) ;{ }^{1} \mathrm{H} \mathrm{NMR}\left(250.13 \mathrm{MHz}, \mathrm{CDCl}_{3}\right) \delta(\mathrm{ppm}): 0.94(\mathrm{t}, J=$ $7.4 \mathrm{~Hz}, 3 \mathrm{H}), 1.22(\mathrm{~d}, J=6.9 \mathrm{~Hz}, 3 \mathrm{H}), 1.55-1.66(\mathrm{~m}, 2 \mathrm{H}), 3.39-3.53(\mathrm{~m}, 1 \mathrm{H}), 3.47$ (s, $3 \mathrm{H}), 4.71(\mathrm{~s}, 1 \mathrm{H}), 7.32-7.45(\mathrm{~m}, 5 \mathrm{H}) ;{ }^{13} \mathrm{C} \mathrm{NMR}\left(62.89 \mathrm{MHz}, \mathrm{CDCl}_{3}\right) \delta(\mathrm{ppm}): 11.4$, 20.5, 29.6, 40.0, 58.0, 89.0, 127.0, 128.6, 136.4, 200.4; HRMS (ESI): m/z calcd for $\mathrm{C}_{13} \mathrm{H}_{18} \mathrm{O}_{2} \mathrm{SNa}[\mathrm{M}+\mathrm{Na}]^{+}: 261.0920$, found: 261.0920 .

(R)-MPA thioester of $(S)$-octane-2-thiol [(R)-MPA-6]

$[\alpha]_{\mathrm{D}}=-9.29\left(c=1.03, \mathrm{CHCl}_{3}\right) ;{ }^{1} \mathrm{H} \mathrm{NMR}\left(250.13 \mathrm{MHz}, \mathrm{CDCl}_{3}\right) \delta(\mathrm{ppm}): 0.84(\mathrm{t}, J=6.7$ $\mathrm{Hz}, 3 \mathrm{H}), 1.20$ (br s, 8H), 1.29 (d, $J=6.9 \mathrm{~Hz}, 3 \mathrm{H}), 1.46-1.56(\mathrm{~m}, 2 \mathrm{H}), 3.46$ (s, 3H), 3.46$3.57(\mathrm{~m}, 1 \mathrm{H}), 4.71(\mathrm{~s}, 1 \mathrm{H}), 7.32-7.45(\mathrm{~m}, 5 \mathrm{H}) ;{ }^{13} \mathrm{C} \mathrm{NMR}\left(62.89 \mathrm{MHz}, \mathrm{CDCl}_{3}\right) \delta(\mathrm{ppm})$ : 14.0, 21.3, 22.5, 26.8, 29.0, 31.6, 36.5, 38.5, 57.9, 89.0, 127.0, 128.5, 128.6, 136.5, 200.4; HRMS (FAB+): m/z calcd for $\mathrm{C}_{17} \mathrm{H}_{27} \mathrm{O}_{2} \mathrm{~S}[\mathrm{M}+\mathrm{H}]^{+}:$295.1732, found: 294.1730.

(S)-MPA thioester of $(S)$-octane-2-thiol [(S)-MPA-6]

$[\alpha]_{\mathrm{D}}=+10.64\left(c=1.01, \mathrm{CHCl}_{3}\right) ;{ }^{1} \mathrm{H}-\mathrm{NMR}\left(250.13 \mathrm{MHz}, \mathrm{CDCl}_{3}\right) \delta(\mathrm{ppm}): 0.87(\mathrm{t}, \mathrm{J}=6.5$ $\mathrm{Hz}, 3 \mathrm{H}), 1.22$ (d, J = 6.9 Hz, 3H), 1.25 (br s, 8H), 1.50-1.60 (m, 2H), 3.46 (s, 3H), 3.47$3.52(\mathrm{~m}, 1 \mathrm{H}), 4.71(\mathrm{~s}, 1 \mathrm{H}), 7.32-7.45(\mathrm{~m}, 5 \mathrm{H}) ;{ }^{13} \mathrm{C}-\mathrm{NMR}\left(62.89 \mathrm{MHz}, \mathrm{CDCl}_{3}\right) \delta(\mathrm{ppm})$ : 14.0, 21.0, 22.6, 26.9, 29.0, 31.7, 36.6, 38.6, 57.9, 89.0, 127.0, 128.5, 136.5, 200.4; HRMS (FAB+): m/z calcd for $\mathrm{C}_{17} \mathrm{H}_{27} \mathrm{O}_{2} \mathrm{~S}[\mathrm{M}+\mathrm{H}]^{+}: 295.1732$, found: 294.1733.

(R)-MPA thioester of $(1 S, 2 S, 5 R)$-2-isopropyl-5-methylcyclohexanethiol [(R)-MPA7]

$[\alpha]_{\mathrm{D}}=+38.81\left(c=1.03, \mathrm{CHCl}_{3}\right) ;{ }^{1} \mathrm{H}$ NMR $\left(500.13 \mathrm{MHz}, \mathrm{CDCl}_{3}\right) \delta(\mathrm{ppm}): 0.63(\mathrm{~d}, J=$ $6.6 \mathrm{~Hz}, 3 \mathrm{H}), 0.84$ (d, $J=6.5 \mathrm{~Hz}, 3 \mathrm{H}), 0.85$ (d, $J=6.5 \mathrm{~Hz}, 3 \mathrm{H}), 0.86-0.93$ (m, 1H), 0.96$1.04(\mathrm{~m}, 1 \mathrm{H}), 1.08-1.14(\mathrm{~m}, 1 \mathrm{H}), 1.24-1.43$ (m, 2H), 1.60-1.74 (m, 2H), 1.79-1.84 (m, 2H), $3.46(\mathrm{~s}, 3 \mathrm{H}), 4.02-4.04(\mathrm{~m}, 1 \mathrm{H}), 4.74(\mathrm{~s}, 1 \mathrm{H}), 7.30-7.37$ (m, 3H), $7.43(\mathrm{dd}, J=1.4$, $7.9 \mathrm{~Hz}, 2 \mathrm{H}) ;{ }^{13} \mathrm{C}$ NMR (125.76 MHz, $\left.\mathrm{CDCl}_{3}\right) \delta(\mathrm{ppm}): 20.4,20.9,22.1,27.6,28.1$, 
30.5, 35.0, 41.9, 44.1, 47.7, 57.8, 89.1, 127.0, 128.5, 128.6, 136.5, 199.7; HRMS (ESI): $\mathrm{m} / \mathrm{z}$ calcd for $\mathrm{C}_{19} \mathrm{H}_{28} \mathrm{O}_{2} \mathrm{SNa}[\mathrm{M}+\mathrm{Na}]^{+}: 343.1702$, found: 343.1693 .

(S)-MPA thioester of $(1 S, 2 S, 5 R)$-2-isopropyl-5-methylcyclohexanethiol [(S)-MPA7]

$[\alpha]_{\mathrm{D}}=+79.96\left(c=0.97, \mathrm{CHCl}_{3}\right) ;{ }^{1} \mathrm{H}-\mathrm{NMR}\left(500.13 \mathrm{MHz}, \mathrm{CDCl}_{3}\right) \delta(\mathrm{ppm}): 0.79(\mathrm{~d}, \mathrm{~J}=$ 6.6 Hz, 3H), $0.80(\mathrm{~d}, \mathrm{~J}=6.5 \mathrm{~Hz}, 3 \mathrm{H}), 0.84-0.91(\mathrm{~m}, 1 \mathrm{H}), 0.87(\mathrm{~d}, \mathrm{~J}=6.6 \mathrm{~Hz}, 3 \mathrm{H}), 0.95-$ $1.04(\mathrm{~m}, 1 \mathrm{H}), 1.11-1.16(\mathrm{~m}, 1 \mathrm{H}), 1.24-1.29(\mathrm{~m}, 1 \mathrm{H}), 1.37-1.44(\mathrm{~m}, 1 \mathrm{H}), 1.54-1.63(\mathrm{~m}$, 1H), 1.65-1.74 (m, 2H), 1.80-1.85 (m, 1H), $3.46(\mathrm{~s}, 3 \mathrm{H}), 4.07-4.09(\mathrm{~m}, 1 \mathrm{H}), 4.73(\mathrm{~s}$, 1H), 7.32-7.38 (m, 3H), $7.43(\mathrm{dd}, \mathrm{J}=1.3,8.2 \mathrm{~Hz}, 2 \mathrm{H}) ;{ }^{13} \mathrm{C}-\mathrm{NMR}\left(125.76 \mathrm{MHz}, \mathrm{CDCl}_{3}\right)$ $\delta$ (ppm): 20.6, 20.9, 22.1, 27.6, 28.0, 30.5, 35.0, 41.7, 44.1, 47.6, 57.9, 89.2, 127.0, 128.5, 128.6, 136.5, 199.6; HRMS $(\mathrm{FAB}+)$ : $\mathrm{m} / \mathrm{z}$ calcd for $\mathrm{C}_{19} \mathrm{H}_{29} \mathrm{O}_{2} \mathrm{~S}[\mathrm{M}+\mathrm{H}]^{+}$: 321.1888, found: 321.1882 .

\section{$(R)$-MPA thioester of $(R)$-ethyl 2-mercaptopropanoate [(R)-MPA-8]}

$[\alpha]_{\mathrm{D}}=+85.60\left(c=0.96, \mathrm{CHCl}_{3}\right) ;{ }^{1} \mathrm{H} \mathrm{NMR}\left(250.13 \mathrm{MHz}, \mathrm{CDCl}_{3}\right) \delta(\mathrm{ppm}): 1.23(\mathrm{t}, J=$ $7.1 \mathrm{~Hz}, 3 \mathrm{H}), 1.45$ (d, $J=7.3 \mathrm{~Hz}, 3 \mathrm{H}), 3.49$ (s, 3H), 4.06-4.15 (m, 1H), 4.17 (q, $J=7.1$ $\mathrm{Hz}, 2 \mathrm{H}), 4.75$ (s, 1H), 7.33-7.43 (m, 5H); ${ }^{13} \mathrm{C} \mathrm{NMR}\left(62.89 \mathrm{MHz}, \mathrm{CDCl}_{3}\right) \delta(\mathrm{ppm}): 14.0$, 17.3, 40.1, 58.2, 61.5, 88.4, 126.8, 128.6, 135.9, 172.0, 199.4; HRMS (ESI): m/z calcd for $\mathrm{C}_{14} \mathrm{H}_{18} \mathrm{O}_{4} \mathrm{SNa}[\mathrm{M}+\mathrm{Na}]^{+}:$305.0818, found: 305.0818 .

(S)-MPA thioester of $(R)$-ethyl 2-mercaptopropanoate [(S)-MPA-8]

$[\alpha]_{\mathrm{D}}=+103.97\left(c=0.99, \mathrm{CHCl}_{3}\right) ;{ }^{1} \mathrm{H} \mathrm{NMR}\left(250.13 \mathrm{MHz}, \mathrm{CDCl}_{3}\right) \delta(\mathrm{ppm}): 1.12(\mathrm{t}, J=$ $7.1 \mathrm{~Hz}, 3 \mathrm{H}), 1.51$ (d, $J=7.3 \mathrm{~Hz}, 3 \mathrm{H}), 3.47$ (s, 3H), 4.08 (q, $J=7.1 \mathrm{~Hz}, 2 \mathrm{H}), 4.08-4.19$ $(\mathrm{m}, 1 \mathrm{H}), 4.75(\mathrm{~s}, 1 \mathrm{H}), 7.33-7.45(\mathrm{~m}, 5 \mathrm{H}) ;{ }^{13} \mathrm{C} \mathrm{NMR}\left(62.89 \mathrm{MHz}, \mathrm{CDCl}_{3}\right) \delta(\mathrm{ppm}): 13.8$, $17.5,40.0,58.1,61.5,88.6,127.0,128.6,135.8,171.8,199.0 ;$ HRMS (FAB+): m/z calcd for $\mathrm{C}_{14} \mathrm{H}_{19} \mathrm{O}_{4} \mathrm{~S}[\mathrm{M}+\mathrm{H}]^{+}:$283.1004, found: 283.1009 .

(R)-MPA thioester of $(R)$-methyl 2-mercapto-2-phenylacetate [(R)-MPA-9] $[\alpha]_{\mathrm{D}}=-62.40\left(c=1.00, \mathrm{CHCl}_{3}\right) ;{ }^{1} \mathrm{H} \mathrm{NMR}\left(500.13 \mathrm{MHz}, \mathrm{CDCl}_{3}\right) \delta(\mathrm{ppm}): 3.50(\mathrm{~s}, 3 \mathrm{H})$, $3.73(\mathrm{~s}, 3 \mathrm{H}), 4.77(\mathrm{~s}, 1 \mathrm{H}), 5.12(\mathrm{~s}, 1 \mathrm{H}), 7.29-7.37(\mathrm{~m}, 10 \mathrm{H}) ;{ }^{13} \mathrm{C} \mathrm{NMR}(125.76 \mathrm{MHz}$, $\left.\mathrm{CDCl}_{3}\right) \delta(\mathrm{ppm}): 50.4,53.0,58.3,88.3,126.9,128.4,128.6,128.8,128.9,134.1,135.7$, 170.6, 199.9; HRMS (FAB+): m/z calcd for $\mathrm{C}_{18} \mathrm{H}_{19} \mathrm{O}_{4} \mathrm{~S}[\mathrm{M}+\mathrm{H}]^{+}: 331.1004$, found: 331.1007 . 
(S)-MPA thioester of $(R)$-methyl 2-mercapto-2-phenylacetate [(S)-MPA-9]

$[\alpha]_{\mathrm{D}}=-44.84\left(c=0.95, \mathrm{CHCl}_{3}\right) ;{ }^{1} \mathrm{H} \mathrm{NMR}\left(500.13 \mathrm{MHz}, \mathrm{CDCl}_{3}\right) \delta(\mathrm{ppm}): 3.43(\mathrm{~s}, 3 \mathrm{H})$, $3.64(\mathrm{~s}, 3 \mathrm{H}), 4.72(\mathrm{~s}, 1 \mathrm{H}), 5.21(\mathrm{~s}, 1 \mathrm{H}), 7.30-7.43(\mathrm{~m}, 10 \mathrm{H}) ;{ }^{13} \mathrm{C}$ NMR $(125.76 \mathrm{MHz}$, $\left.\mathrm{CDCl}_{3}\right) \delta(\mathrm{ppm}):$ 50.4, 53.0, 58.1, 88.4, 127.2, 128.5, 128.7, 128.8, 128.9, 135.0, 135.7, 170.2, 199.3; HRMS (FAB+): m/z calcd for $\mathrm{C}_{18} \mathrm{H}_{19} \mathrm{O}_{4} \mathrm{~S}[\mathrm{M}+\mathrm{H}]^{+}: 331.1004$, found: 331.1013.

(R)-MPA thioester of 1-thio- $\beta$-D-glucose tetraacetate [(R)-MPA-10]

$[\alpha]_{\mathrm{D}}=-9.27\left(c=1.20, \mathrm{CHCl}_{3}\right) ;{ }^{1} \mathrm{H} \mathrm{NMR}\left(500.13 \mathrm{MHz}, \mathrm{CDCl}_{3}\right) \delta(\mathrm{ppm}): 1.94(\mathrm{~s}, 3 \mathrm{H})$, 1.96 (s, 3H), 1.99 (s, 3H), 2.01 (s, 3H), 3.44 (s, 3H), 3.76 (ddd, $J=2.1,4.7,9.7 \mathrm{~Hz}, 1 \mathrm{H})$, $3.99(\mathrm{dd}, J=2.1,12.4 \mathrm{~Hz}, 1 \mathrm{H}), 4.24$ (dd, $J=4.8,12.4 \mathrm{~Hz}, 1 \mathrm{H}), 4.75$ (s, 1H), 5.08 (dd, $J$ $=9.1,9.7 \mathrm{~Hz}, 1 \mathrm{H}), 5.13-5.15(\mathrm{~m}, 1 \mathrm{H}), 5.18(\mathrm{~d}, J=10.1 \mathrm{~Hz}, 1 \mathrm{H}), 5.25(\mathrm{dd}, J=9.0,9.2$ $\mathrm{Hz}, 1 \mathrm{H}), 7.35-7.41(\mathrm{~m}, 5 \mathrm{H}) ;{ }^{13} \mathrm{C} \mathrm{NMR}\left(125.76 \mathrm{MHz}, \mathrm{CDCl}_{3}\right) \delta$ (ppm): 20.4, 20.5, 20.6, 58.0, 61.6, 68.0, 69.4, 74.0, 76.4, 79.2, 88.5, 127.1, 128.7, 129.0, 135.3, 169.2, 169.3, 170.1, 170.5, 197.4; HRMS (FAB+): m/z calcd for $\mathrm{C}_{23} \mathrm{H}_{29} \mathrm{O}_{11} \mathrm{~S}[\mathrm{M}+\mathrm{H}]^{+}:$513.1431, found: 513.1429 .

\section{(S)-MPA thioester of 1-thio- $\beta$-D-glucose tetraacetate [(S)-MPA-10]}

$[\alpha]_{\mathrm{D}}=-17.39\left(c=1.15, \mathrm{CHCl}_{3}\right) ;{ }^{1} \mathrm{H} \mathrm{NMR}\left(500.13 \mathrm{MHz}, \mathrm{CDCl}_{3}\right) \delta(\mathrm{ppm}): 1.70(\mathrm{~s}, 3 \mathrm{H})$, 1.97 (s, 3H), 2.02 (s, 3H), 2.08 (s, 3H), 3.49 (s, 3H), 3.80 (ddd, $J=2.2,4.4,10.0 \mathrm{~Hz}$, $1 \mathrm{H}), 4.11(\mathrm{dd}, J=2.1,12.5 \mathrm{~Hz}, 1 \mathrm{H}), 4.26(\mathrm{dd}, J=4.4,12.5 \mathrm{~Hz}, 1 \mathrm{H}), 4.78(\mathrm{~s}, 1 \mathrm{H}), 5.08$ (dd, $J=9.5,9.9 \mathrm{~Hz}, 1 \mathrm{H}), 5.12-5.14(\mathrm{~m}, 2 \mathrm{H}), 5.20$ (ddd, $J=1.2,7.9,9.2 \mathrm{~Hz}, 1 \mathrm{H}), 7.33-$ 7.40 (m, 5H); ${ }^{13} \mathrm{C}$ NMR (125.76 MHz, $\left.\mathrm{CDCl}_{3}\right) \delta(\mathrm{ppm}): 20.2,20.5,20.7,58.4,61.7$, $67.9,69.5,73.9,76.5,79.3,88.3,126.7,128.6,128.9$, 135.6, 169.1, 169.3, 170.0, 170.6, 198.1; HRMS (FAB+): m/z calcd for $\mathrm{C}_{23} \mathrm{H}_{29} \mathrm{O}_{11} \mathrm{~S}[\mathrm{M}+\mathrm{H}]^{+}:$513.1431, found: 513.1426.

(R)-MPA thioester of $\left(3^{\prime} R, 4 S\right)$-3-(1'-oxobutyl-3'-mercapto)-4-isopropyl-5,5dimethyl-1,3-oxazolidinone [(R)-MPA-11]

${ }^{1} \mathrm{H}$ NMR (300.13 MHz, $\mathrm{CDCl}_{3}$ ) $\delta(\mathrm{ppm}): 0.93$ (d, $\left.J=6.9 \mathrm{~Hz}, 3 \mathrm{H}\right), 1.00$ (d, $J=6.9 \mathrm{~Hz}$, $3 \mathrm{H}), 1.33(\mathrm{~d}, J=6.6 \mathrm{~Hz}, 3 \mathrm{H}), 1.38(\mathrm{~s}, 3 \mathrm{H}), 1.50(\mathrm{~s}, 3 \mathrm{H}), 2.07-2.18(\mathrm{~m}, 1 \mathrm{H}), 3.21(\mathrm{dd}, J$ $=6.9,17.1 \mathrm{~Hz}, 1 \mathrm{H}), 3.31(\mathrm{dd}, J=6.3,17.1 \mathrm{~Hz}, 1 \mathrm{H}), 3.46(\mathrm{~s}, 3 \mathrm{H}), 3.99(\mathrm{dd}, J=6.9,13.8$ $\mathrm{Hz}, 1 \mathrm{H}), 4.13(\mathrm{~d}, J=3.3 \mathrm{~Hz}, 1 \mathrm{H}), 4.71(\mathrm{~s}, 1 \mathrm{H}), 7.31-7.43(\mathrm{~m}, 5 \mathrm{H}) ;{ }^{13} \mathrm{C}$ NMR $(75.47$ $\left.\mathrm{MHz}, \mathrm{CDCl}_{3}\right) \delta(\mathrm{ppm}): 17.1,21.0,21.4,21.5,28.8,29.5,29.7,34.0,41.8,58.1,66.3$, 83.0, 88.8, 127.0, 128.5, 136.2, 153.4, 170.8, 200.0. 
(S)-MPA thioester of $\left(3^{\prime} R, 4 S\right)$-3-(1'-oxobutyl-3'-mercapto)-4-isopropyl-5,5dimethyl-1,3-oxazolidinone [(S)-MPA-11]

${ }^{1} \mathrm{H}$ NMR (300.13 MHz, $\mathrm{CDCl}_{3}$ ) $\delta(\mathrm{ppm}): 0.90$ (d, $\left.J=6.9 \mathrm{~Hz}, 3 \mathrm{H}\right), 0.97$ (d, $J=6.9 \mathrm{~Hz}$, $3 \mathrm{H}), 1.25(\mathrm{~s}, 3 \mathrm{H}), 1.40(\mathrm{~d}, J=6.6 \mathrm{~Hz}, 3 \mathrm{H}), 1.48(\mathrm{~s}, 3 \mathrm{H}), 2.04-2.17(\mathrm{~m}, 1 \mathrm{H}), 3.21(\mathrm{~d}, J=$ $6.6 \mathrm{~Hz}, 2 \mathrm{H}), 3.45(\mathrm{~s}, 3 \mathrm{H}), 4.01(\mathrm{dd}, J=6.6,13.0 \mathrm{~Hz}, 1 \mathrm{H}), 4.05(\mathrm{~d}, J=3.3 \mathrm{~Hz}, 1 \mathrm{H}), 4.71$ (s, 1H), 7.30-7.41 (m, 5H); $\left.{ }^{13} \mathrm{C} \mathrm{NMR} \mathrm{(75.47} \mathrm{MHz,} \mathrm{CDCl}_{3}\right) \delta(\mathrm{ppm}): 17.0,21.2,21.4$, 28.7, 29.5, 29.6, 34.0, 41.6, 58.0, 66.3, 83.0, 88.8, 127.1, 128.5, 128.6, 136.1, 153.4, $170.7,200.6$.

(R)-MPA thioester of $\quad\left(2^{\prime} S, 3^{\prime} R, 4 S\right)$-3-(1'-oxobutyl-2-methyl-3'-mercapto)-4isopropyl-5,5-dimethyl-1,3-oxazolidinone [(R)-MPA-12]

${ }^{1} \mathrm{H}$ NMR (300.13 MHz, $\mathrm{CDCl}_{3}$ ) $\delta(\mathrm{ppm}): 0.96$ (d, $\left.J=6.9 \mathrm{~Hz}, 3 \mathrm{H}\right), 1.00$ (d, $J=6.9 \mathrm{~Hz}$, $3 \mathrm{H}), 1.20(\mathrm{~d}, J=7.2 \mathrm{~Hz}, 3 \mathrm{H}), 1.27$ (d, $J=6.9 \mathrm{~Hz}, 3 \mathrm{H}), 1.35(\mathrm{~s}, 3 \mathrm{H}), 1.50$ (s, 3H), 2.08$2.18(\mathrm{~m}, 1 \mathrm{H}), 3.43(\mathrm{~s}, 3 \mathrm{H}), 3.91(\mathrm{dq}, J=6.6,6.9 \mathrm{~Hz}, 1 \mathrm{H}), 4.14(\mathrm{~d}, J=3.0 \mathrm{~Hz}, 1 \mathrm{H}), 4.17$ $(\mathrm{dq}, J=6.6,6.9 \mathrm{~Hz}, 1 \mathrm{H}), 4.72(\mathrm{~s}, 1 \mathrm{H}), 7.28-7.44(\mathrm{~m}, 5 \mathrm{H}) ;{ }^{13} \mathrm{C} \mathrm{NMR}(75.47 \mathrm{MHz}$, $\left.\mathrm{CDCl}_{3}\right) \delta(\mathrm{ppm}): 15.4,17.0,19.2,21.3,21.5,28.8,29.5,29.6,40.3,41.8,58.0,66.5$, $82.7,88.9,127.0,128.5,136.4,153.1,175.2,199.9$.

(S)-MPA thioester of $\left(2^{\prime} S, 3\right.$ ' $\left.R, 4 S\right)$-3-(1'-oxobutyl-2-methyl-3'-mercapto)-4isopropyl-5,5-dimethyl-1,3-oxazolidinone [(S)-MPA-12]

${ }^{1} \mathrm{H}$ NMR (300.13 MHz, $\mathrm{CDCl}_{3}$ ) $\delta(\mathrm{ppm}): 0.95$ (d, $\left.J=7.2 \mathrm{~Hz}, 3 \mathrm{H}\right), 0.97$ (d, $J=7.2 \mathrm{~Hz}$, $3 \mathrm{H}), 1.15(\mathrm{~d}, J=6.9 \mathrm{~Hz}, 3 \mathrm{H}), 1.34(\mathrm{~s}, 3 \mathrm{H}), 1.36$ (d, $J=7.2 \mathrm{~Hz}, 3 \mathrm{H}), 1.50$ (s, 3H), 2.06$2.17(\mathrm{~m}, 1 \mathrm{H}), 3.45(\mathrm{~s}, 3 \mathrm{H}), 3.88(\mathrm{dq}, J=6.6,6.9 \mathrm{~Hz}, 1 \mathrm{H}), 4.11(\mathrm{~d}, J=3.0 \mathrm{~Hz}, 1 \mathrm{H}), 4.17$ $(\mathrm{dq}, J=6.6,6.9 \mathrm{~Hz}, 1 \mathrm{H}), 4.74(\mathrm{~s}, 1 \mathrm{H}), 7.31-7.43(\mathrm{~m}, 5 \mathrm{H}) ;{ }^{13} \mathrm{C}$ NMR $(75.47 \mathrm{MHz}$, $\left.\mathrm{CDCl}_{3}\right) \delta(\mathrm{ppm}): 15.7,17.0,19.8,21.3,21.5,28.8,29.5,29.7,40.4,41.8,58.0,66.5$, $82.7,88.9,127.0,129.0,136.3,153.1,175.2,200.0$.

(R)-MPA thioester of (3'S,4S)-3-(1'-oxobutyl-3'-mercapto-3'-phenyl)-4-isopropyl5,5-dimethyl-1,3-oxazolidinone [(R)-MPA-13]

${ }^{1} \mathrm{H}$ NMR (300.13 MHz, $\left.\mathrm{CDCl}_{3}\right) \delta(\mathrm{ppm}): 0.90$ (d, $\left.J=6.9 \mathrm{~Hz}, 3 \mathrm{H}\right), 0.95$ (d, $J=6.9 \mathrm{~Hz}$, $3 \mathrm{H}), 1.11(\mathrm{~s}, 3 \mathrm{H}), 1.44(\mathrm{~s}, 3 \mathrm{H}), 2.01-2.12(\mathrm{~m}, 1 \mathrm{H}), 3.45(\mathrm{~s}, 3 \mathrm{H}), 3.48(\mathrm{dd}, J=6.0,16.8$ $\mathrm{Hz}, 1 \mathrm{H}), 3.94(\mathrm{dd}, J=9.0,16.8 \mathrm{~Hz}, 1 \mathrm{H}), 3.98$ (d, $J=3.3 \mathrm{~Hz}, 1 \mathrm{H}), 4.72(\mathrm{~s}, 1 \mathrm{H}), 5.08$ $(\mathrm{dd}, J=6.0,9.3 \mathrm{~Hz}, 1 \mathrm{H}), 7.15-7.40(\mathrm{~m}, 10 \mathrm{H}) ;{ }^{13} \mathrm{C} \mathrm{NMR}\left(75.47 \mathrm{MHz}, \mathrm{CDCl}_{3}\right) \delta(\mathrm{ppm})$ : $17.0,21.3,28.4,29.4,29.6,41.0,43.0,58.1,66.3,83.0,88.7,127.0,127.6,127.8$, $128.5,128.6,128.7,136.0,140.0,153.5,170.3,199.2$. 
(S)-MPA thioester of (3'S,4S)-3-(1'-oxobutyl-3'-mercapto-3'-phenyl)-4-isopropyl5,5-dimethyl-1,3-oxazolidinone [(S)-MPA-13]

${ }^{1} \mathrm{H}$ NMR (300.13 MHz, $\mathrm{CDCl}_{3}$ ) $\delta(\mathrm{ppm}): 0.87$ (d, $\left.J=6.9 \mathrm{~Hz}, 3 \mathrm{H}\right), 0.92$ (d, $J=6.9 \mathrm{~Hz}$, $3 \mathrm{H}), 1.02(\mathrm{~s}, 3 \mathrm{H}), 1.42(\mathrm{~s}, 3 \mathrm{H}), 1.98-2.09(\mathrm{~m}, 1 \mathrm{H}), 3.34$ (dd, $J=6.3,16.5 \mathrm{~Hz}, 1 \mathrm{H}), 3.41$ (s, 3H), $3.91(\mathrm{~d}, J=3.3 \mathrm{~Hz}, 1 \mathrm{H}), 3.92(\mathrm{dd}, J=9.0,16.5 \mathrm{~Hz}, 1 \mathrm{H}), 4.70$ (s, 1H), 5.10 (dd, $J=6.3,9.0 \mathrm{~Hz}, 1 \mathrm{H}), 7.20-7.41(\mathrm{~m}, 10 \mathrm{H}) ;{ }^{13} \mathrm{C} \mathrm{NMR}\left(75.47 \mathrm{MHz}, \mathrm{CDCl}_{3}\right) \delta(\mathrm{ppm}): 17.0$, 21.3, 28.2, 29.4, 29.6, 40.6, 43.0, 58.1, 66.3, 83.0, 88.6, 127.0, 127.6, 127.8, 127.9, $128.6,128.7,136.0,140.4,153.4,170.0,199.2$.

(R)-MPA thioester of 3-(3-oxo-7 $\alpha$-thio-17 $\beta$-hydroxy-4-androsten-17 $\alpha$-yl)propionic acid $\gamma$-lactone [(R)-MPA-14]

$[\alpha]_{\mathrm{D}}=-56.95\left(c=1.80, \mathrm{CHCl}_{3}\right) ;{ }^{1} \mathrm{H} \mathrm{NMR}\left(500.13 \mathrm{MHz}, \mathrm{CDCl}_{3}\right) \delta(\mathrm{ppm}): 0.97(\mathrm{~s}, 3 \mathrm{H})$, 1.09-1.14 (m, 1H), 1.20 (s, 3H), 1.26-1.52 (m, 4H), 1.55-1.58 (m, 1H), 1.64-1.81 (m, $6 \mathrm{H}), 1.99-2.07(\mathrm{~m}, 2 \mathrm{H}), 2.21(\mathrm{dd}, J=2.6,14.9 \mathrm{~Hz}, 1 \mathrm{H}), 2.26-2.32(\mathrm{~m}, 1 \mathrm{H}), 2.37-2.42$ (m, 2H), 2.43-2.58 (m, 2H), 2.77 (ddd, $J=1.7,4.1,14.7 \mathrm{~Hz}, 1 \mathrm{H}), 3.41$ (s, 3H), 3.95$3.97(\mathrm{~m}, 1 \mathrm{H}), 4.74(\mathrm{~s}, 1 \mathrm{H}), 5.53(\mathrm{~s}, 1 \mathrm{H}), 7.37-7.40(\mathrm{~m}, 5 \mathrm{H}) ;{ }^{13} \mathrm{C} \mathrm{NMR}(125.76 \mathrm{MHz}$, $\left.\mathrm{CDCl}_{3}\right) \delta(\mathrm{ppm}): 14.5,17.6,20.4,22.2,29.1,31.0,31.1,33.8,35.0,35.5,38.4,38.8$, 39.5, 43.8, 45.3, 45.9, 49.2, 57.4, 89.0, 95.4, 126.8, 127.1, 128.8, 129.0, 135.9, 165.4, 176.5, 198.4, 198.5; HRMS (CI): m/z calcd for $\mathrm{C}_{31} \mathrm{H}_{39} \mathrm{O}_{5} \mathrm{~S}[\mathrm{M}+\mathrm{H}]^{+}: 523.2518$, found: 523.2519 .

(S)-MPA thioester of 3-(3-oxo-7 $\alpha$-thio-17 $\beta$-hydroxy-4-androsten-17 $\alpha$-yl)propionic acid $\gamma$-lactone $[(S)$-MPA-14]

$[\alpha]_{\mathrm{D}}=+8.61\left(c=1.95, \mathrm{CHCl}_{3}\right) ;{ }^{1} \mathrm{H} \operatorname{NMR}\left(500.13 \mathrm{MHz}, \mathrm{CDCl}_{3}\right) \delta(\mathrm{ppm}): 0.91(\mathrm{~s}, 3 \mathrm{H})$, 0.96-1.00 (m, 1H), 1.07-1.12 (m, 1H), 1.17-1.21 (m, 1H), $1.22(\mathrm{~s}, 3 \mathrm{H}), 1.26-1.49(\mathrm{~m}$, $4 \mathrm{H}), 1.51-1.55(\mathrm{~m}, 1 \mathrm{H}), 1.62-1.67(\mathrm{~m}, 2 \mathrm{H}), 1.74(\mathrm{dt}, J=5.2,13.6 \mathrm{~Hz}, 1 \mathrm{H}), 1.96(\mathrm{dt}, J=$ 3.4, $10.9 \mathrm{~Hz}, 1 \mathrm{H}), 1.99-2.08$ (m, 2H), 2.19-2.25 (m, 1H), 2.38-2.55 (m, 5H), 2.88 (ddd, $J=1.8,4.3,14.7 \mathrm{~Hz}, 1 \mathrm{H}), 3.48(\mathrm{~s}, 3 \mathrm{H}), 3.93-3.96(\mathrm{~m}, 1 \mathrm{H}), 4.77(\mathrm{~s}, 1 \mathrm{H}), 5.71(\mathrm{~s}, 1 \mathrm{H})$, 7.33-7.37 (m, 3H), $7.43(\mathrm{dd}, J=1.7,7.6 \mathrm{~Hz}, 2 \mathrm{H}) ;{ }^{13} \mathrm{C} \mathrm{NMR}\left(125.76 \mathrm{MHz}, \mathrm{CDCl}_{3}\right) \delta$ (ppm): 14.4, 17.6, 20.4, 22.2, 29.1, 30.9, 31.1, 33.8, 34.8, 35.5, 38.4, 39.1, 40.0, 43.5, 45.2, 45.9, 49.2, 58.3, 88.8, 95.4, 126.4, 126.7, 128.5, 136.3, 165.6, 176.5, 198.7, 199.5; HRMS (CI): $\mathrm{m} / \mathrm{z}$ calcd for $\mathrm{C}_{31} \mathrm{H}_{39} \mathrm{O}_{5} \mathrm{~S}[\mathrm{M}+\mathrm{H}]^{+}:$523.2518, found: 523.2521. 


\section{(R)-MPA thioester of thiocholesterol [(R)-MPA-15]}

$[\alpha]_{\mathrm{D}}=-12.40\left(c=1.00, \mathrm{CHCl}_{3}\right) ;{ }^{1} \mathrm{H} \mathrm{NMR}\left(500.13 \mathrm{MHz}, \mathrm{CDCl}_{3}\right) \delta(\mathrm{ppm}): 0.66(\mathrm{~s}, 3 \mathrm{H})$, $0.86(\mathrm{~d}, J=6.6 \mathrm{~Hz}, 6 \mathrm{H}), 0.90(\mathrm{~d}, J=6.5 \mathrm{~Hz}, 3 \mathrm{H}), 0.93-1.28(\mathrm{~m}, 13 \mathrm{H}), 1.32-1.68(\mathrm{~m}$, $11 \mathrm{H}), 1.78-2.00(\mathrm{~m}, 5 \mathrm{H}), 2.18(\mathrm{dd}, J=2.4,13.5 \mathrm{~Hz}, 1 \mathrm{H}), 2.25-2.31(\mathrm{~m}, 1 \mathrm{H}), 3.25-3.31$ (m, 1H), $3.46(\mathrm{~s}, 3 \mathrm{H}), 4.70(\mathrm{~s}, 1 \mathrm{H}), 5.30(\mathrm{~d}, J=5.0 \mathrm{~Hz}, 1 \mathrm{H}), 7.31-7.38(\mathrm{~m}, 3 \mathrm{H}), 7.43$ (d, $J=6.9 \mathrm{~Hz}, 2 \mathrm{H}) ;{ }^{13} \mathrm{C} \mathrm{NMR}\left(125.76 \mathrm{MHz}, \mathrm{CDCl}_{3}\right) \delta$ (ppm): 11.9, 18.7, 19.2, 20.9, 22.6, 22.8, 23.8, 24.2, 28.0, 28.2, 29.1, 31.8, 35.8, 36.2, 36.6, 38.6, 39.5, 39.7, 42.3, 42.5, 50.1, 56.1, 56.7, 57.9, 88.9, 121.6, 127.0, 128.6, 136.3, 141.0, 200.0; HRMS (FAB+): $\mathrm{m} / \mathrm{z}$ calcd for $\mathrm{C}_{36} \mathrm{H}_{55} \mathrm{O}_{2} \mathrm{~S}[\mathrm{M}+\mathrm{H}]^{+}:$551.3923, found: 551.3932 .

\section{(S)-MPA thioester of thiocholesterol [(S)-MPA-15]}

$[\alpha]_{\mathrm{D}}=-60.56\left(c=1.80, \mathrm{CHCl}_{3}\right) ;{ }^{1} \mathrm{H} \mathrm{NMR}\left(500.13 \mathrm{MHz}, \mathrm{CDCl}_{3}\right) \delta(\mathrm{ppm}): 0.66(\mathrm{~s}, 3 \mathrm{H})$, $0.86(\mathrm{~d}, J=6.6 \mathrm{~Hz}, 6 \mathrm{H}), 0.90(\mathrm{~d}, J=6.5 \mathrm{~Hz}, 3 \mathrm{H}), 0.93-1.19(\mathrm{~m}, 12 \mathrm{H}), 1.21-1.29$ (m, $1 \mathrm{H}), 1.32-1.62(\mathrm{~m}, 11 \mathrm{H}), 1.73-1.76(\mathrm{~m}, 1 \mathrm{H}), 1.80-1.85(\mathrm{~m}, 2 \mathrm{H}), 1.94-2.00(\mathrm{~m}, 2 \mathrm{H})$, 2.26-2.36 (m, 2H), 3.24-3.32 (m, 1H), $3.47(\mathrm{~s}, 3 \mathrm{H}), 4.71(\mathrm{~s}, 1 \mathrm{H}), 5.35(\mathrm{~d}, \mathrm{~J}=4.7 \mathrm{~Hz}$, 1H), 7.31-7.38 (m, 3H), $7.42(\mathrm{~d}, J=6.9 \mathrm{~Hz}, 2 \mathrm{H}) ;{ }^{13} \mathrm{C} \mathrm{NMR}\left(125.76 \mathrm{MHz}, \mathrm{CDCl}_{3}\right) \delta$ (ppm): 11.8, 18.7, 19.2, 20.8, 22.5, 22.8, 23.8, 24.2, 28.0, 28.2, 28.8, 31.8, 35.8, 36.2, 36.6, 38.9, 39.5, 39.7, 42.3, 42.5, 50.1, 56.1, 56.7, 58.0, 88.9, 121.6, 126.9, 128.6, 136.4, 141.1, 200.1; HRMS (FAB+): $\mathrm{m} / \mathrm{z}$ calcd for $\mathrm{C}_{36} \mathrm{H}_{55} \mathrm{O}_{2} \mathrm{~S}[\mathrm{M}+\mathrm{H}]^{+}:$551.3923, found: 551.3929 .

\section{(R)-PTBA thioester of $(S)$-butane-2-thiol [(R)-PTBA-5]}

$[\alpha]_{\mathrm{D}}=+6.28\left(c=0.35, \mathrm{CHCl}_{3}\right) ;{ }^{1} \mathrm{H} \mathrm{NMR}\left(250.13 \mathrm{MHz}, \mathrm{CDCl}_{3}\right) \delta(\mathrm{ppm}): 0.85(\mathrm{t}, J=7.4$ $\mathrm{Hz}, 3 \mathrm{H}), 1.27(\mathrm{~s}, 9 \mathrm{H}), 1.28(\mathrm{~d}, J=6.9 \mathrm{~Hz}, 3 \mathrm{H}), 1.45-1.56(\mathrm{~m}, 2 \mathrm{H}), 3.28-3.42(\mathrm{~m}, 1 \mathrm{H})$, 4.99 (s, 1H), 7.24-7.37 (m, 3H), 7.48 (dd, $J=1.7,8.1 \mathrm{~Hz}, 2 \mathrm{H}) ;{ }^{13} \mathrm{C}$ NMR $(62.89 \mathrm{MHz}$, $\left.\mathrm{CDCl}_{3}\right) \delta(\mathrm{ppm}): 11.4,20.7,28.0,29.5,39.8,77.2,79.7,126.5,127.8,128.2,139.3$, 203.5; HRMS (ESI): $\mathrm{m} / \mathrm{z}$ calcd for $\mathrm{C}_{16} \mathrm{H}_{24} \mathrm{O}_{2} \mathrm{SNa}[\mathrm{M}+\mathrm{Na}]^{+}: 303.1389$, found: 303.1385.

\section{(S)-PTBA thioester of $(S)$-butane-2-thiol [(S)-PTBA-5]}

$[\alpha]_{\mathrm{D}}=+4.10\left(c=0.50, \mathrm{CHCl}_{3}\right) ;{ }^{1} \mathrm{H} \mathrm{NMR}\left(250.13 \mathrm{MHz}, \mathrm{CDCl}_{3}\right) \delta(\mathrm{ppm}): 0.95(\mathrm{t}, J=7.4$ $\mathrm{Hz}, 3 \mathrm{H}), 1.18$ (d, $J=7.0 \mathrm{~Hz}, 3 \mathrm{H}), 1.27$ (s, 9H), 1.54-1.65 (m, 2H), 3.30-3.43 (m, 1H), 4.99 (s, 1H), 7.24-7.37 (m, 3H), 7.48 (dd, $J=1.7,8.2 \mathrm{~Hz}, 2 \mathrm{H}) ;{ }^{13} \mathrm{C}$ NMR (62.89 MHz, $\left.\mathrm{CDCl}_{3}\right) \delta(\mathrm{ppm}): 11.5,20.5,28.0,29.6,39.9,77.2,79.6,126.5,127.8,128.2,139.3$, 203.6; HRMS (ESI): $\mathrm{m} / \mathrm{z}$ calcd for $\mathrm{C}_{16} \mathrm{H}_{24} \mathrm{O}_{2} \mathrm{SNa}[\mathrm{M}+\mathrm{Na}]^{+}:$303.1389, found: 303.1387. 
(R)-2-NTBA thioester of $(S)$-butane-2-thiol [(R)-2-NTBA-5]

$[\alpha]_{\mathrm{D}}=+10.00\left(c=0.20, \mathrm{CHCl}_{3}\right) ;{ }^{1} \mathrm{H} \mathrm{NMR}\left(250.13 \mathrm{MHz}, \mathrm{CDCl}_{3}\right) \delta(\mathrm{ppm}): 0.83(\mathrm{t}, J=$ $7.4 \mathrm{~Hz}, 3 \mathrm{H}), 1.29$ (d, $J=6.9 \mathrm{~Hz}, 3 \mathrm{H}), 1.30$ (s, 9H), 1.45-1.55 (m, 2H), 3.32-3.39 (m, 1H), 5.15 (s, 1H), 7.43-7.49 (m, 2H), 7.61 (dd, $J=1.5,8.5 \mathrm{~Hz}, 1 \mathrm{H}), 7.79-7.86(\mathrm{~m}, 3 \mathrm{H})$, $7.94(\mathrm{~s}, 1 \mathrm{H}) ;{ }^{13} \mathrm{C}$ NMR (62.89 MHz, $\left.\mathrm{CDCl}_{3}\right) \delta(\mathrm{ppm}): 11.4,20.7,28.1,29.7,39.9,77.2$, $79.9,124.4,125.6,125.9,126.0,127.6,128.0,128.1,133.1,133.2,136.9,203.4$; HRMS (ESI): $\mathrm{m} / \mathrm{z}$ calcd for $\mathrm{C}_{20} \mathrm{H}_{26} \mathrm{O}_{2} \mathrm{~S}[\mathrm{M}+\mathrm{H}]^{+}: 353.1546$, found: 353.1548 .

\section{(S)-2-NTBA thioester of $(S)$-butane-2-thiol [(S)-2-NTBA-5]}

$[\alpha]_{\mathrm{D}}=+7.33\left(c=0.30, \mathrm{CHCl}_{3}\right) ;{ }^{1} \mathrm{H}$ NMR $\left(250.13 \mathrm{MHz}, \mathrm{CDCl}_{3}\right) \delta(\mathrm{ppm}): 0.95(\mathrm{t}, J=7.4$ $\mathrm{Hz}, 3 \mathrm{H}), 1.16(\mathrm{~d}, J=6.9 \mathrm{~Hz}, 3 \mathrm{H}), 1.31(\mathrm{~s}, 9 \mathrm{H}), 1.54-1.66(\mathrm{~m}, 2 \mathrm{H}), 3.33-3.41(\mathrm{~m}, 1 \mathrm{H})$, $5.16(\mathrm{~s}, 1 \mathrm{H}), 7.43-7.50(\mathrm{~m}, 2 \mathrm{H}), 7.61(\mathrm{dd}, J=1.7,8.5 \mathrm{~Hz}, 1 \mathrm{H}), 7.80-7.87(\mathrm{~m}, 3 \mathrm{H}), 7.94$ $(\mathrm{s}, 1 \mathrm{H}) ;{ }^{13} \mathrm{C} \mathrm{NMR}\left(62.89 \mathrm{MHz}, \mathrm{CDCl}_{3}\right) \delta(\mathrm{ppm}): 11.5,20.5,28.1,29.6,40.0,77.2,79.8$, $124.4,125.6,125.9,126.0,127.6,128.0,128.1,133.0,133.2,136.8,203.4$; HRMS (ESI): $\mathrm{m} / \mathrm{z}$ calcd for $\mathrm{C}_{20} \mathrm{H}_{26} \mathrm{O}_{2} \mathrm{~S}[\mathrm{M}+\mathrm{H}]^{+}: 353.1546$, found: 353.1548 .

\section{(R)-2-NTBA thioester of $(S)$-octane-2-thiol [(R)-2-NTBA-6]}

$[\alpha]_{\mathrm{D}}=+9.25\left(c=0.80, \mathrm{CHCl}_{3}\right) ;{ }^{1} \mathrm{H} \mathrm{NMR}\left(250.13 \mathrm{MHz}, \mathrm{CDCl}_{3}\right) \delta(\mathrm{ppm}): 0.78(\mathrm{t}, J=6.6$ Hz, 3H), 1.13 (br s, 8H), 1.28 (d, $J=6.9$ Hz, 3H), 1.31 (s, 9H), 1.40-1.50 (m, 2H), 3.33$3.47(\mathrm{~m}, 1 \mathrm{H}), 5.16(\mathrm{~s}, 1 \mathrm{H}), 7.42-7.50(\mathrm{~m}, 2 \mathrm{H}), 7.61(\mathrm{dd}, J=1.5,8.5 \mathrm{~Hz}, 1 \mathrm{H}), 7.79-7.87$ $(\mathrm{m}, 3 \mathrm{H}), 7.94(\mathrm{~s}, 1 \mathrm{H}) ;{ }^{13} \mathrm{C} \mathrm{NMR}\left(62.89 \mathrm{MHz}, \mathrm{CDCl}_{3}\right) \delta(\mathrm{ppm}): 14.0,21.3,22.5,26.8$, 28.0, 29.0, 31.6, 36.5, 38.5, 77.2, 79.9, 124.4, 125.6, 125.9, 126.0, 127.6, 128.0, 128.1, 133.0, 133.1, 136.8, 203.4; HRMS (ESI): $\mathrm{m} / \mathrm{z}$ calcd for $\mathrm{C}_{24} \mathrm{H}_{34} \mathrm{O}_{2} \mathrm{SNa}[\mathrm{M}+\mathrm{Na}]^{+}$: 409.2172, found: 409.2169 .

(S)-2-NTBA thioester of $(S)$-octane-2-thiol [(S)-2-NTBA-6]

$[\alpha]_{\mathrm{D}}=-7.55\left(c=0.90, \mathrm{CHCl}_{3}\right) ;{ }^{1} \mathrm{H} \mathrm{NMR}\left(250.13 \mathrm{MHz}, \mathrm{CDCl}_{3}\right) \delta(\mathrm{ppm}): 0.87(\mathrm{t}, J=6.6$ $\mathrm{Hz}, 3 \mathrm{H}), 1.16$ (d, J=6.9 Hz, 3H), 1.26 (br s, 8H), 1.31 (s, 9H), 1.50-1.60 (m, 2H), 3.35$3.49(\mathrm{~m}, 1 \mathrm{H}), 5.16(\mathrm{~s}, 1 \mathrm{H}), 7.42-7.50(\mathrm{~m}, 2 \mathrm{H}), 7.61(\mathrm{dd}, J=1.6,8.5 \mathrm{~Hz}, 1 \mathrm{H}), 7.80-7.87$ $(\mathrm{m}, 3 \mathrm{H}), 7.94(\mathrm{~s}, 1 \mathrm{H}) ;{ }^{13} \mathrm{C} \mathrm{NMR}\left(62.89 \mathrm{MHz}, \mathrm{CDCl}_{3}\right) \delta(\mathrm{ppm}): 14.1,20.9,22.6,27.0$, 28.0, 29.0, 31.7, 36.6, 38.5, 77.2, 79.8, 124.4, 125.5, 125.9, 126.0, 127.6, 127.9, 128.1, 133.0, 133.1, 136.8, 203.4; HRMS (ESI): $\mathrm{m} / \mathrm{z}$ calcd for $\mathrm{C}_{24} \mathrm{H}_{34} \mathrm{O}_{2} \mathrm{SNa}[\mathrm{M}+\mathrm{Na}]^{+}$: 409.2172, found: 409.2163 . 
$(R)$-2-NTBA thioester of $(1 S, 2 S, 5 R)$-2-isopropyl-5-methylcyclohexanethiol $[(R)-2$ NTBA-7]

$[\alpha]_{\mathrm{D}}=+65.75\left(c=0.80, \mathrm{CHCl}_{3}\right) ;{ }^{1} \mathrm{H} \mathrm{NMR}\left(500.13 \mathrm{MHz}, \mathrm{CDCl}_{3}\right) \delta(\mathrm{ppm}): 0.56(\mathrm{~d}, J=$ $6.5 \mathrm{~Hz}, 3 \mathrm{H}), 0.78(\mathrm{~d}, J=6.6 \mathrm{~Hz}, 3 \mathrm{H}), 0.86(\mathrm{~d}, J=6.4 \mathrm{~Hz}, 3 \mathrm{H}), 0.86-0.94(\mathrm{~m}, 1 \mathrm{H})$, 0.967-1.12 (m, 2H), 1.28-1.38 (m, 2H), $1.32(\mathrm{~s}, 9 \mathrm{H}), 1.60-1.68(\mathrm{~m}, 1 \mathrm{H}), 1.70-1.77$ (m, 1H), 1.78-1.85 (m, 2H), 3.95-3.97 (m, 1H), 5.19 (s, 1H), 7.43-7.49 (m, 2H), 7.60 (dd, $J$ $=1.7,8.5 \mathrm{~Hz}, 1 \mathrm{H}), 7.78-7.85(\mathrm{~m}, 3 \mathrm{H}), 7.93(\mathrm{~s}, 1 \mathrm{H}) ;{ }^{13} \mathrm{C} \mathrm{NMR}\left(125.76 \mathrm{MHz}, \mathrm{CDCl}_{3}\right) \delta$ (ppm): 20.4, 20.9, 22.2, 27.6, 28.1, 30.4, 35.1, 42.0, 44.2, 47.8, 76.5, 80.2, 124.4, 125.6, 125.9, 126.0, 127.6, 127.9, 128.1, 133.1, 133.2, 136.7, 202.7; HRMS (ESI): m/z calcd for $\mathrm{C}_{26} \mathrm{H}_{36} \mathrm{O}_{2} \mathrm{SNa}[\mathrm{M}+\mathrm{Na}]^{+}$: 435.2328, found: 435.2329 .

(S)-2-NTBA thioester of $(1 S, 2 S, 5 R)$-2-isopropyl-5-methylcyclohexanethiol $[(S)$-2NTBA-7]

$[\alpha]_{\mathrm{D}}=+8.11\left(c=1.40, \mathrm{CHCl}_{3}\right) ;{ }^{1} \mathrm{H} \mathrm{NMR}\left(500.13 \mathrm{MHz}, \mathrm{CDCl}_{3}\right) \delta(\mathrm{ppm}): 0.73(\mathrm{~d}, J=6.3$ $\mathrm{Hz}, 3 \mathrm{H}), 0.82-0.85(\mathrm{~m}, 1 \mathrm{H}), 0.83$ (d, $J=6.6 \mathrm{~Hz}, 3 \mathrm{H}), 0.90$ (d, $J=6.6 \mathrm{~Hz}, 3 \mathrm{H}), 0.97-1.07$ $(\mathrm{m}, 1 \mathrm{H}), 1.10-1.17(\mathrm{~m}, 1 \mathrm{H}), 1.17-1.26(\mathrm{~m}, 1 \mathrm{H}), 1.32(\mathrm{~s}, 9 \mathrm{H}), 1.39-1.48(\mathrm{~m}, 1 \mathrm{H}), 1.56-$ $1.59(\mathrm{~m}, 1 \mathrm{H}), 1.57-1.62(\mathrm{~m}, 1 \mathrm{H}), 1.66-172(\mathrm{~m}, 1 \mathrm{H}), 1.80-1.86(\mathrm{~m}, 1 \mathrm{H}), 4.00-4.03(\mathrm{~m}$, $1 \mathrm{H}), 5.17$ (s, 1H), 7.44-7.49 (m, 2H), $7.61(\mathrm{dd}, J=1.7,8.5 \mathrm{~Hz}, 1 \mathrm{H}), 7.80-7.86(\mathrm{~m}, 3 \mathrm{H})$, $7.95(\mathrm{~s}, 1 \mathrm{H}) ;{ }^{13} \mathrm{C}$ NMR $\left(125.76 \mathrm{MHz}, \mathrm{CDCl}_{3}\right) \delta(\mathrm{ppm}): 20.6,21.0,22.1,27.7,27.9$, 28.0, 30.7, 35.1, 41.6, 43.9, 47.7, 76.5, 80.0, 124.4, 125.5, 125.9, 126.0, 127.6, 127.9, 128.1, 133.2, 133.2, 136.7, 202.8; HRMS (ESI): $\mathrm{m} / \mathrm{z}$ calcd for $\mathrm{C}_{26} \mathrm{H}_{36} \mathrm{O}_{2} \mathrm{SNa}[\mathrm{M}+\mathrm{Na}]^{+}$: 435.2328, found: 435.2333 .

(R)-2-NTBA thioester of $(R)$-ethyl 2-mercaptopropanoate [(R)-2-NTBA-8]

$[\alpha]_{\mathrm{D}}=+76.75\left(c=0.80, \mathrm{CHCl}_{3}\right) ;{ }^{1} \mathrm{H} \mathrm{NMR}\left(250.13 \mathrm{MHz}, \mathrm{CDCl}_{3}\right) \delta(\mathrm{ppm}): 1.23(\mathrm{t}, J=$ $7.1 \mathrm{~Hz}, 3 \mathrm{H}), 1.32(\mathrm{~s}, 9 \mathrm{H}), 1.41$ (d, $J=7.3 \mathrm{~Hz}, 3 \mathrm{H}), 4.02$ (q, $J=7.3 \mathrm{~Hz}, 1 \mathrm{H}), 4.17$ (q, $J=$ $7.1 \mathrm{~Hz}, 2 \mathrm{H}), 5.20$ (s, 1H), 7.43-7.51 (m, 2H), 7.59 (dd, $J=1.6,8.5 \mathrm{~Hz}, 1 \mathrm{H}), 7.80-7.87$ $(\mathrm{m}, 3 \mathrm{H}), 7.94(\mathrm{~s}, 1 \mathrm{H}) ;{ }^{13} \mathrm{C} \mathrm{NMR}\left(62.89 \mathrm{MHz}, \mathrm{CDCl}_{3}\right) \delta(\mathrm{ppm}):$ 14.0, 17.1, 28.0, 40.3, $61.4,77.2,79.3,124.2,125.6,126.1,127.6,128.1,133.1,136.2,172.5,202.2$; HRMS (ESI): $\mathrm{m} / \mathrm{z}$ calcd for $\mathrm{C}_{21} \mathrm{H}_{26} \mathrm{O}_{4} \mathrm{SNa}[\mathrm{M}+\mathrm{Na}]^{+}: 397.1444$, found: 397.1453 .

(S)-2-NTBA thioester of $(R)$-ethyl 2-mercaptopropanoate [(S)-2-NTBA-8]

$[\alpha]_{\mathrm{D}}=+68.54\left(c=1.10, \mathrm{CHCl}_{3}\right) ;{ }^{1} \mathrm{H} \mathrm{NMR}\left(250.13 \mathrm{MHz}, \mathrm{CDCl}_{3}\right) \delta(\mathrm{ppm}): 1.01(\mathrm{t}, J=$ $7.1 \mathrm{~Hz}, 3 \mathrm{H}), 1.31(\mathrm{~s}, 9 \mathrm{H}), 1.50(\mathrm{~d}, J=7.3 \mathrm{~Hz}, 3 \mathrm{H}), 4.01(\mathrm{q}, J=7.1 \mathrm{~Hz}, 2 \mathrm{H}), 4.06$ (q, $J=$ $7.3 \mathrm{~Hz}, 1 \mathrm{H}), 5.19$ (s, 1H), 7.43-7.51 (m, 2H), 7.60 (dd, $J=1.6,8.5 \mathrm{~Hz}, 1 \mathrm{H}), 7.80-7.87$ 
(m, 3H), $7.94(\mathrm{~s}, 1 \mathrm{H}) ;{ }^{13} \mathrm{C} \mathrm{NMR}\left(62.89 \mathrm{MHz}, \mathrm{CDCl}_{3}\right) \delta(\mathrm{ppm}):$ 13.7, 17.6, 28.0, 40.1, 61.4, 77.2, 79.4, 124.3, 125.8, 126.1, 127.6, 128.1, 133.1, 136.2, 172.1, 201.9; HRMS (ESI): $\mathrm{m} / \mathrm{z}$ calcd for $\mathrm{C}_{21} \mathrm{H}_{26} \mathrm{O}_{4} \mathrm{SNa}[\mathrm{M}+\mathrm{Na}]^{+}: 397.1444$, found: 397.1449 .

(R)-2-NTBA thioester of $(R)$-methyl 2-mercapto-2-phenylacetate [(R)-2-NTBA-9] $[\alpha]_{\mathrm{D}}=-55.40\left(c=1.00, \mathrm{CHCl}_{3}\right) ;{ }^{1} \mathrm{H}$ NMR $\left(500.13 \mathrm{MHz}, \mathrm{CDCl}_{3}\right) \delta(\mathrm{ppm}): 1.33(\mathrm{~s}, 9 \mathrm{H})$, $3.73(\mathrm{~s}, 3 \mathrm{H}), 5.03(\mathrm{~s}, 1 \mathrm{H}), 5.22(\mathrm{~s}, 1 \mathrm{H}), 7.26-7.28(\mathrm{~m}, 2 \mathrm{H}), 7.31-7.35(\mathrm{~m}, 3 \mathrm{H}), 7.45-7.49$ $(\mathrm{m}, 2 \mathrm{H}), 7.55(\mathrm{dd}, J=1.6,8.5 \mathrm{~Hz}, 1 \mathrm{H}), 7.80-7.87(\mathrm{~m}, 3 \mathrm{H}), 7.89(\mathrm{~s}, 1 \mathrm{H}) ;{ }^{13} \mathrm{C} \mathrm{NMR}$ (125.76 MHz, $\left.\mathrm{CDCl}_{3}\right) \delta(\mathrm{ppm}):$ 28.0, 50.9, 52.9, 77.2, 79.3, 124.2, 125.6, 126.1, 127.6, 128.1, 128.4, 128.8, 133.0, 133.1, 134.2, 136.0, 170.9, 202.6; HRMS (ESI): m/z calcd for $\mathrm{C}_{25} \mathrm{H}_{26} \mathrm{O}_{4} \mathrm{SNa}[\mathrm{M}+\mathrm{Na}]^{+}:$445.1444, found: 445.1451 .

\section{$(S)$-2-NTBA thioester of $(R)$-methyl 2-mercapto-2-phenylacetate [(S)-2-NTBA-9]} $[\alpha]_{\mathrm{D}}=-20.60\left(c=0.16, \mathrm{CHCl}_{3}\right) ;{ }^{1} \mathrm{H} \mathrm{NMR}\left(500.13 \mathrm{MHz}, \mathrm{CDCl}_{3}\right) \delta(\mathrm{ppm}): 1.26(\mathrm{~s}, 9 \mathrm{H})$, $3.56(\mathrm{~s}, 3 \mathrm{H}), 5.14(\mathrm{~s}, 1 \mathrm{H}), 5.16(\mathrm{~s}, 1 \mathrm{H}), 7.30-7.36(\mathrm{~m}, 3 \mathrm{H}), 7.43(\mathrm{dd}, J=1.6,8.0 \mathrm{~Hz}$, 2H), 7.46-7.50 (m, 2H), $7.60(\mathrm{dd}, J=1.7,8.5 \mathrm{~Hz}, 1 \mathrm{H}), 7.80-7.86(\mathrm{~m}, 3 \mathrm{H}), 7.93(\mathrm{~s}, 1 \mathrm{H})$; ${ }^{13} \mathrm{C} \mathrm{NMR}\left(125.76 \mathrm{MHz}, \mathrm{CDCl}_{3}\right) \delta(\mathrm{ppm}): 28.0,50.7,52.8,77.1,79.4,124.4,125.9$, $126.1,127.7,128.2,128.5,128.8,133.1,133.2,135.4,136.3,170.4,201.9$; HRMS (ESI): $\mathrm{m} / \mathrm{z}$ calcd for $\mathrm{C}_{25} \mathrm{H}_{26} \mathrm{O}_{4} \mathrm{SNa}[\mathrm{M}+\mathrm{Na}]^{+}:$445.1444, found: 445.1449.

\section{$(R)$-2-NTBA thioester of 1-thio- $\beta$-D-glucose tetraacetate [(R)-2-NTBA-10]}

$[\alpha]_{\mathrm{D}}=+20.29\left(c=0.70, \mathrm{CHCl}_{3}\right) ;{ }^{1} \mathrm{H} \mathrm{NMR}\left(500.13 \mathrm{MHz}, \mathrm{CDCl}_{3}\right) \delta(\mathrm{ppm}): 1.28(\mathrm{~s}, 9 \mathrm{H})$, 1.89 (s, 3H), 1.97 (s, 3H), 1.99 (s, 3H), 2.00 (s, 3H), 3.71 (ddd, $J=2.2,4.6,10.1 \mathrm{~Hz}$, 1H), 3.93 (dd, $J=2.2,12.4 \mathrm{~Hz}, 1 \mathrm{H}), 4.19$ (dd, $J=4.6,12.4 \mathrm{~Hz}, 1 \mathrm{H}), 5.07$ (dd, $J=9.4$, $9.8 \mathrm{~Hz}, 1 \mathrm{H}), 5.14(\mathrm{dd}, J=0.5,9.9 \mathrm{~Hz}, 1 \mathrm{H}), 5.17$ (dd, $J=8.4,10.1 \mathrm{~Hz}, 1 \mathrm{H}), 5.18(\mathrm{~s}, 1 \mathrm{H})$, $5.25(\mathrm{dd}, J=8.5,9.3 \mathrm{~Hz}, 1 \mathrm{H}), 7.45-7.50(\mathrm{~m}, 2 \mathrm{H}), 7.57$ (dd, $J=1.7,8.5 \mathrm{~Hz}, 1 \mathrm{H}), 7.80-$ 7.86 (m, 3H), 7.92 (s, 1H); ${ }^{13} \mathrm{C}$ NMR (125.76 MHz, $\left.\mathrm{CDCl}_{3}\right) \delta$ (ppm): 20.4, 20.5, 20.6, $27.9,61.6,68.0,69.6,74.1,76.3,77.1,79.3,79.4,124.2,125.8,126.2,127.7,128.1$, 128.3, 133.1, 133.2, 135.6, 169.1, 169.3, 170.1, 170.5, 200.4; HRMS (ESI): m/z calcd for $\mathrm{C}_{30} \mathrm{H}_{36} \mathrm{O}_{11} \mathrm{SNa}[\mathrm{M}+\mathrm{Na}]^{+}: 627.1871$, found: 627.1871 .

$(S)$-2-NTBA thioester of 1-thio- $\beta$-D-glucose tetraacetate $[(S)$-2-NTBA-10]

$[\alpha]_{\mathrm{D}}=-24.80\left(c=0.75, \mathrm{CHCl}_{3}\right) ;{ }^{1} \mathrm{H} \mathrm{NMR}\left(500.13 \mathrm{MHz}, \mathrm{CDCl}_{3}\right) \delta(\mathrm{ppm}): 1.30(\mathrm{~s}, 9 \mathrm{H})$, $1.41(\mathrm{~s}, 3 \mathrm{H}), 1.94$ (s, 3H), 2.00 (s, 3H), 2.10 (s, 3H), 3.78 (ddd, $J=2.0,4.1,8.8 \mathrm{~Hz}, 1 \mathrm{H})$, $4.10(\mathrm{dd}, J=2.2,12.4 \mathrm{~Hz}, 1 \mathrm{H}), 4.28(\mathrm{dd}, J=4.4,12.4 \mathrm{~Hz}, 1 \mathrm{H}), 5.04-5.17$ (m, 4H), 5.23 (s, 1H), 7.44-7.51 (m, 2H), $7.55(\mathrm{dd}, J=1.7,8.5 \mathrm{~Hz}, 1 \mathrm{H}), 7.79-7.85$ (m, 3H), 7.91 (s, 
$1 \mathrm{H}) ;{ }^{13} \mathrm{C} \mathrm{NMR}\left(125.76 \mathrm{MHz}, \mathrm{CDCl}_{3}\right) \delta(\mathrm{ppm}): 19.9,20.5,20.7,28.0,61.8,67.9,69.7$, $74.1,76.5,77.3,79.4,79.7,123.9,125.6,126.3,127.6,128.0,128.2,133.1,133.2$, 135.9, 169.2, 169.4, 170.0, 170.6, 200.5; HRMS (ESI): $\mathrm{m} / \mathrm{z}$ calcd for $\mathrm{C}_{30} \mathrm{H}_{36} \mathrm{O}_{11} \mathrm{SNa}[\mathrm{M}$ $+\mathrm{Na}]^{+}:$627.1871, found: 627.1873 .

(R)-2-NTBA thioester of 3-(3-oxo-7 $\alpha$-thio-17 $\beta$-hydroxy-4-androsten-17 $\alpha$ yl)propionic acid $\gamma$-lactone $[(R)-2-N T B A-14]$

$[\alpha]_{\mathrm{D}}=-78.50\left(c=0.80, \mathrm{CHCl}_{3}\right) ;{ }^{1} \mathrm{H} \mathrm{NMR}\left(500.13 \mathrm{MHz}, \mathrm{CDCl}_{3}\right) \delta(\mathrm{ppm}): 0.93(\mathrm{~s}, 3 \mathrm{H})$, $1.07(\mathrm{dt}, J=4.3,11.8,11.8 \mathrm{~Hz}, 1 \mathrm{H}), 1.18(\mathrm{~s}, 3 \mathrm{H}), 1.22-1.39$ (m, 14H), 1.41- 1.49 (m, 2H), 1.51-1.54 (m, 1H), 1.62-1.66 (m, 1H), $1.74(\mathrm{dt}, J=5.8,13.4,13.5 \mathrm{~Hz}, 1 \mathrm{H}), 1.97$ $(\mathrm{dt}, J=3.5,11.0,11.1 \mathrm{~Hz}, 1 \mathrm{H}), 2.02-2.12(\mathrm{~m}, 3 \mathrm{H}), 2.20(\mathrm{dd}, J=2.5,14.8 \mathrm{~Hz}, 1 \mathrm{H})$, 2.33-2.44 (m, 4H), 2.77 (ddd, $J=1.7,4.4,14.7 \mathrm{~Hz}, 1 \mathrm{H}), 3.94-3.96(\mathrm{~m}, 1 \mathrm{H}), 5.18$ (s, 1H), 5.56 (s, 1H), 7.46-7.51 (m, 2H), $7.58(\mathrm{dd}, J=1.6,8.5 \mathrm{~Hz}, 1 \mathrm{H}), 7.83-7.86(\mathrm{~m}, 3 \mathrm{H})$, $7.90(\mathrm{~s}, 1 \mathrm{H}) ;{ }^{13} \mathrm{C}$ NMR $\left(125.76 \mathrm{MHz}, \mathrm{CDCl}_{3}\right) \delta(\mathrm{ppm}): 14.5,17.6,20.4,22.2,28.1$, 29.1, 30.6, 31.1, 33.9, 34.9, 35.5, 38.4, 38.9, 39.7, 43.8, 45.2, 46.1, 49.4, 76.6, 80.4, 95.3, 124.4, 126.0, 126.3, 126.7, 127.7, 128.1, 128.3, 133.1, 133.2, 136.1, 165.5, 176.5, 198.6, 200.8; HRMS (ESI): $\mathrm{m} / \mathrm{z}$ calcd for $\mathrm{C}_{38} \mathrm{H}_{47} \mathrm{O}_{5} \mathrm{~S}[\mathrm{M}+\mathrm{H}]^{+}:$615.3139, found: 615.3133 .

(S)-2-NTBA thioester of 3-(3-oxo-7 $\alpha$-thio-17 $\beta$-hydroxy-4-androsten-17 $\alpha$ yl)propionic acid $\gamma$-lactone $[(S)$-2-NTBA-14]

$[\alpha]_{\mathrm{D}}=+23.56\left(c=1.15, \mathrm{CHCl}_{3}\right) ;{ }^{1} \mathrm{H} \mathrm{NMR}\left(500.13 \mathrm{MHz}, \mathrm{CDCl}_{3}\right) \delta(\mathrm{ppm}): 0.56-0.74(\mathrm{~m}$, 3H), 0.79 (s, 3H), 0.86 (dt, $J=6.9,11.2,11.4 \mathrm{~Hz}, 1 \mathrm{H}), 0.95-1.10(\mathrm{~m}, 3 \mathrm{H}), 1.19$ (s, 3H), 1.33 (s, 9H), 1.36-1.42 (m, 2H), 1.56-1.60 (m, 1H), 1.66-1.78 (m, 3H), 1.85 (dt, $J=3.5$, 11.2, 11.2 Hz, 1H), 1.95-2.05 (m, 2H), 2.07-2.14 (m, 1H), 2.35-2.47 (m, 3H), 2.89 (ddd, $J=1.8,4.5,14.5 \mathrm{~Hz}, 1 \mathrm{H}), 3.87-3.90(\mathrm{~m}, 1 \mathrm{H}), 5.28(\mathrm{~s}, 1 \mathrm{H}), 5.75(\mathrm{~s}, 1 \mathrm{H}), 7.46-7.49$ (m, 2H), $7.61(\mathrm{dd}, J=1.7,8.6 \mathrm{~Hz}, 1 \mathrm{H}), 7.79$ (d, $J=8.6 \mathrm{~Hz}, 1 \mathrm{H}), 7.81-7.86(\mathrm{~m}, 2 \mathrm{H}), 8.01$ (s, $1 \mathrm{H}) ;{ }^{13} \mathrm{C} \mathrm{NMR}\left(125.76 \mathrm{MHz}, \mathrm{CDCl}_{3}\right) \delta(\mathrm{ppm}): 14.4,17.5,20.3,22.2,27.9,28.9,29.8$, $30.8,33.9,34.0,35.5,38.5,39.3,40.4,43.4,44.8,45.5,49.0,77.1,79.7,95.1,123.8$, $124.8,126.3,126.4,127.6,127.8,128.1,133.0,133.1,136.0,166.1,176.6,198.9$, 202.4; HRMS (ESI): m/z calcd for $\mathrm{C}_{38} \mathrm{H}_{47} \mathrm{O}_{5} \mathrm{~S}[\mathrm{M}+\mathrm{H}]^{+}:$615.3139, found: 615.3126.

(R)-2-NTBA thioester of thiocholesterol [(R)-2-NTBA-15]

$[\alpha]_{\mathrm{D}}=+30.00\left(c=0.90, \mathrm{CHCl}_{3}\right) ;{ }^{1} \mathrm{H} \mathrm{NMR}\left(500.13 \mathrm{MHz}, \mathrm{CDCl}_{3}\right) \delta(\mathrm{ppm}): 0.66(\mathrm{~s}, 3 \mathrm{H})$, $0.86(\mathrm{~d}, J=6.6 \mathrm{~Hz}, 6 \mathrm{H}), 0.91-1.28(\mathrm{~m}, 18 \mathrm{H}), 1.31(\mathrm{~s}, 9 \mathrm{H}), 1.32-1.66(\mathrm{~m}, 9 \mathrm{H}), 1.77-1.93$ 
(m, 4H), 1.97-2.00 (m, 1H), 2.11 (dd, $J=2.8,13.5 \mathrm{~Hz}, 1 \mathrm{H}), 2.22-2.27$ (m, 1H), 3.16$3.23(\mathrm{~m}, 1 \mathrm{H}), 5.14(\mathrm{~s}, 1 \mathrm{H}), 5.25(\mathrm{~d}, J=4.8 \mathrm{~Hz}, 1 \mathrm{H}), 7.44-7.49(\mathrm{~m}, 2 \mathrm{H}), 7.61(\mathrm{dd}, J=$ 1.7, 8.5 Hz, 1H), 7.80-7.86 (m, 3H), $7.94(\mathrm{~s}, 1 \mathrm{H}) ;{ }^{13} \mathrm{C} \mathrm{NMR}\left(125.76 \mathrm{MHz}, \mathrm{CDCl}_{3}\right) \delta$ (ppm): 11.8, 18.7, 19.3, 20.9, 22.5, 22.8, 23.8, 24.2, 28.0, 28.1, 28.2, 29.1, 31.8, 35.8, 36.2, 36.6, 38.5, 39.5, 39.6, 39.7, 42.3, 42.5, 50.1, 56.1, 56.7, 76.6, 79.8, 121.4, 124.4, 125.6, 126.0, 127.6, 128.0, 128.1, 133.1, 136.8, 141.1, 202.9; HRMS (ESI): m/z calcd for $\mathrm{C}_{43} \mathrm{H}_{63} \mathrm{O}_{2} \mathrm{~S}[\mathrm{M}+\mathrm{H}]^{+}:$643.4543, found: 643.4553 .

(S)-2-NTBA thioester of thiocholesterol [(S)-2-NTBA-15]

$[\alpha]_{\mathrm{D}}=-66.80\left(c=1.00, \mathrm{CHCl}_{3}\right) ;{ }^{1} \mathrm{H} \mathrm{NMR}\left(500.13 \mathrm{MHz}, \mathrm{CDCl}_{3}\right) \delta(\mathrm{ppm}): 0.66(\mathrm{~s}, 3 \mathrm{H})$, $0.86(\mathrm{~d}, J=6.6 \mathrm{~Hz}, 6 \mathrm{H}), 0.88(\mathrm{~d}, J=6.5 \mathrm{~Hz}, 3 \mathrm{H}), 0.92-1.26(\mathrm{~m}, 15 \mathrm{H}), 1.31(\mathrm{~s}, 9 \mathrm{H})$, 1.32-1.60 (m, 9H), 1.65-1.69 (m, 1H), 1.78-1.88 (m, 2H), 1.92-2.00 (m, 2H), 2.28-2.34 (m, 2H), 3.15-3.23 (m, 1H), $5.15(\mathrm{~s}, 1 \mathrm{H}), 5.35$ (d, $J=4.8 \mathrm{~Hz}, 1 \mathrm{H}), 7.43-7.49(\mathrm{~m}, 2 \mathrm{H})$, $7.60(\mathrm{dd}, J=1.7,8.5 \mathrm{~Hz}, 1 \mathrm{H}), 7.80-7.85(\mathrm{~m}, 3 \mathrm{H}), 7.94(\mathrm{~s}, 1 \mathrm{H}) ;{ }^{13} \mathrm{C}$ NMR $(125.76 \mathrm{MHz}$, $\left.\mathrm{CDCl}_{3}\right) \delta$ (ppm): 11.8, 18.7, 19.3, 20.8, 22.6, 22.8, 23.8, 24.3, 28.0, 28.1, 28.2, 28.7, $31.8,31.9,35.8,36.2,36.6,39.0,39.5,39.7,42.3,42.6,50.1,56.1,56.7,77.6,79.8$, $121.4,124.4,125.5,126.0,127.6,128.0,128.1,133.1,133.2,136.8,141.3,203.1$; HRMS (ESI): $\mathrm{m} / \mathrm{z}$ calcd for $\mathrm{C}_{43} \mathrm{H}_{63} \mathrm{O}_{2} \mathrm{~S}[\mathrm{M}+\mathrm{H}]^{+}:$643.4543, found: 643.4547. 
${ }^{1} \mathrm{H}$ NMR and ${ }^{13} \mathrm{C}$ NMR spectra of selected compounds
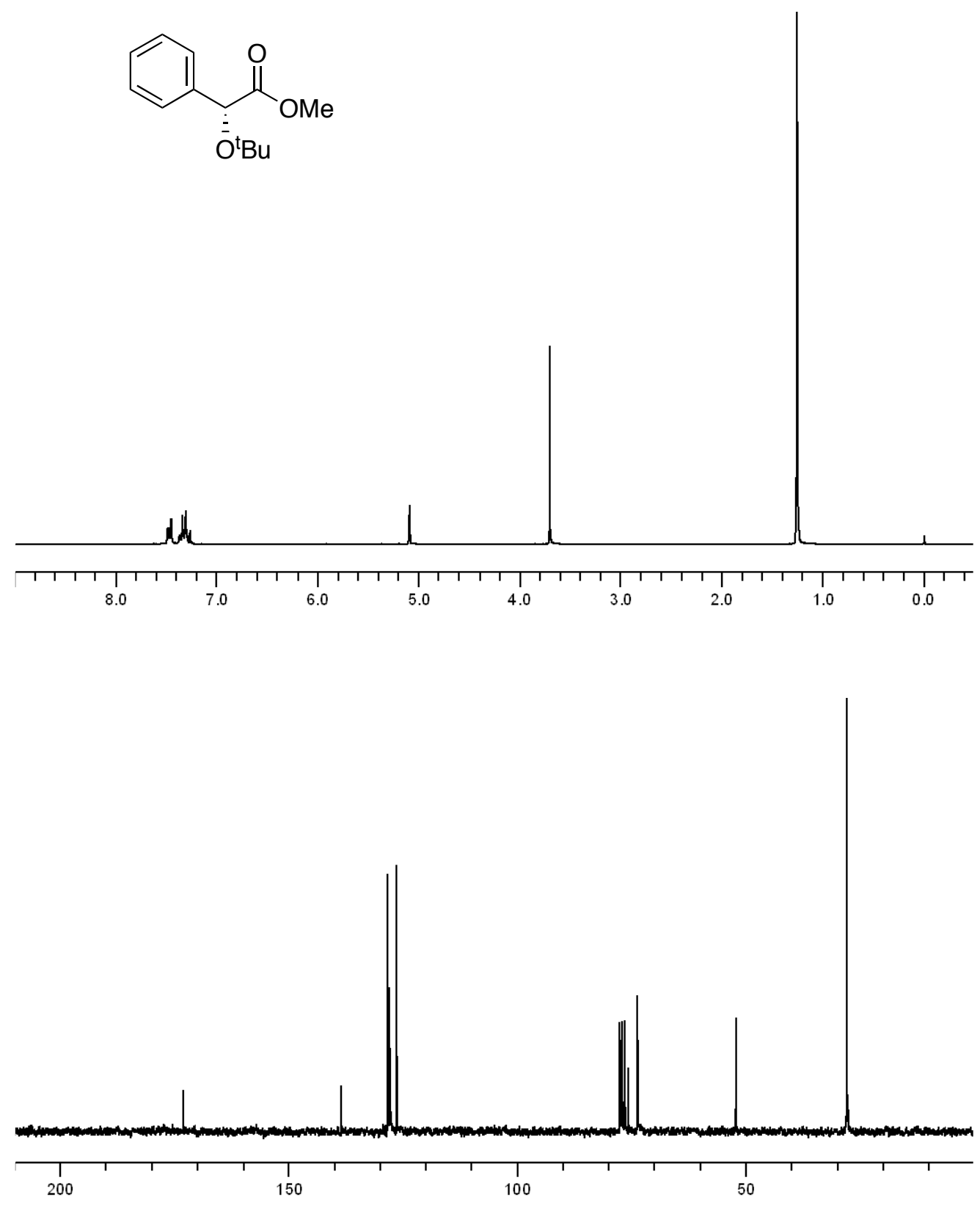

Figure 1S. ${ }^{1} \mathrm{H}$ NMR and ${ }^{13} \mathrm{C}$ NMR spectra of $(R)-16$ a. 

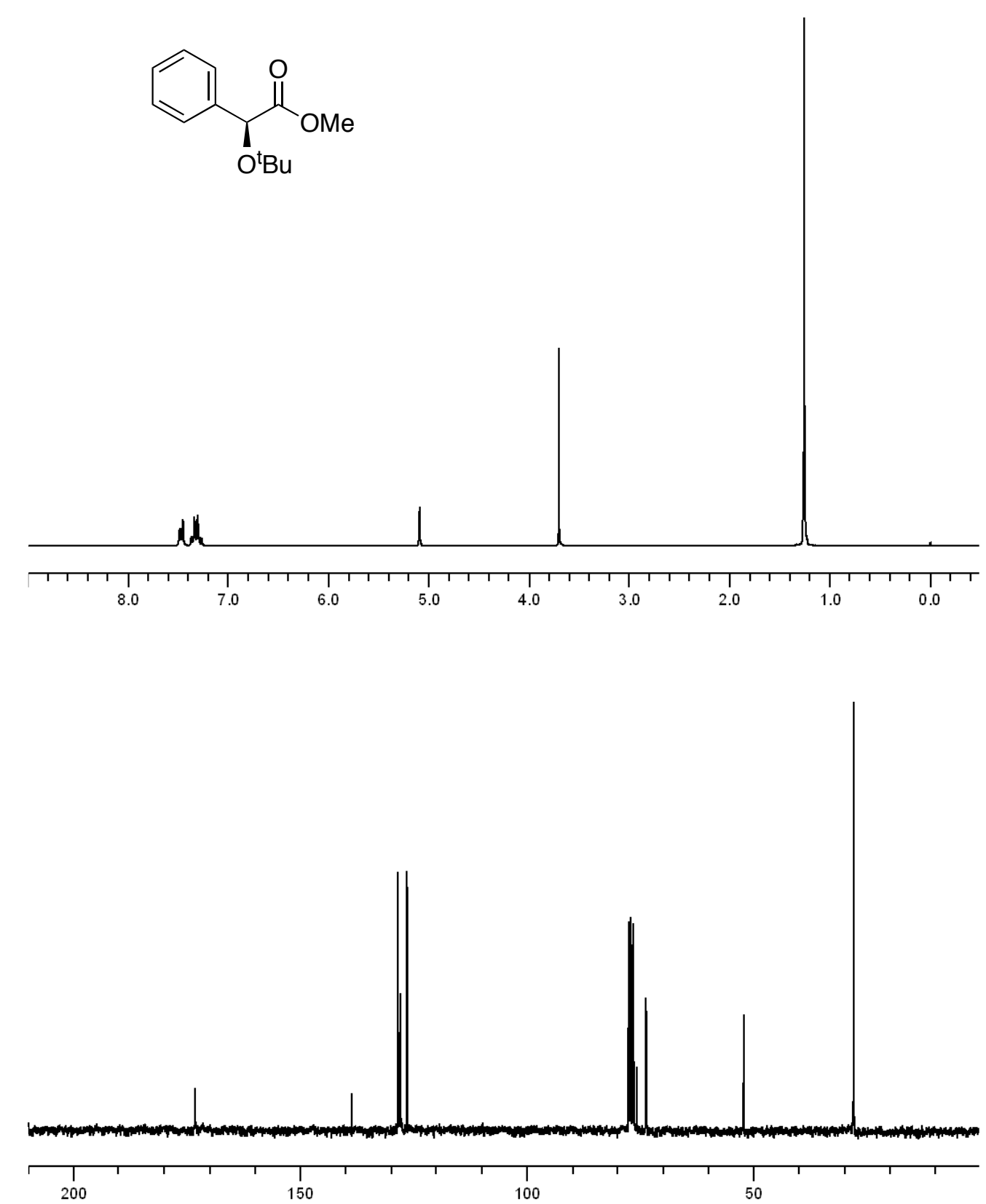

Figure 2S. ${ }^{1} \mathrm{H}$ NMR and ${ }^{13} \mathrm{C}$ NMR spectra of $(S)-16$ a. 

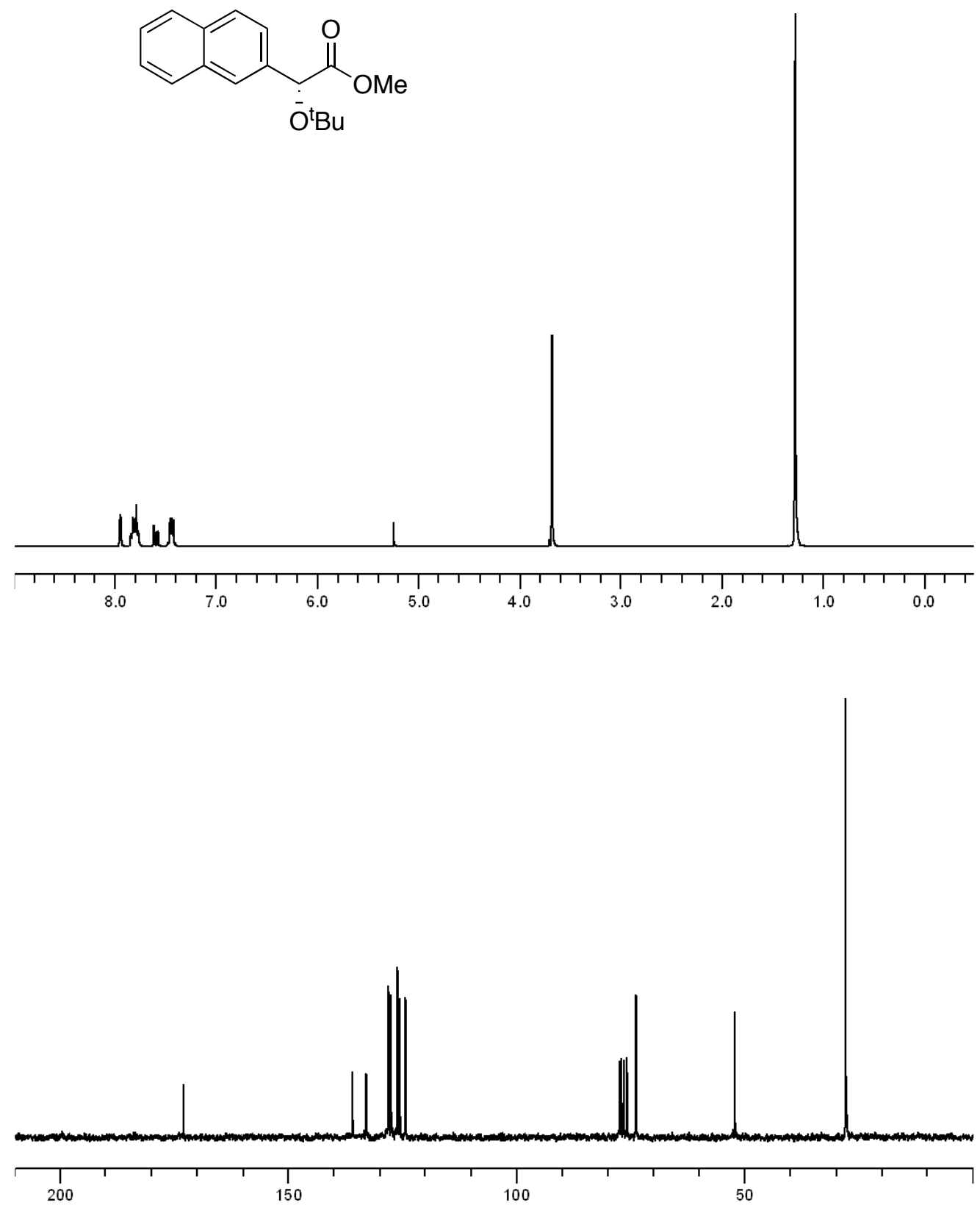

Figure 3S. ${ }^{1} \mathrm{H}$ NMR and ${ }^{13} \mathrm{C}$ NMR spectra of $(R)-17 \mathrm{a}$. 

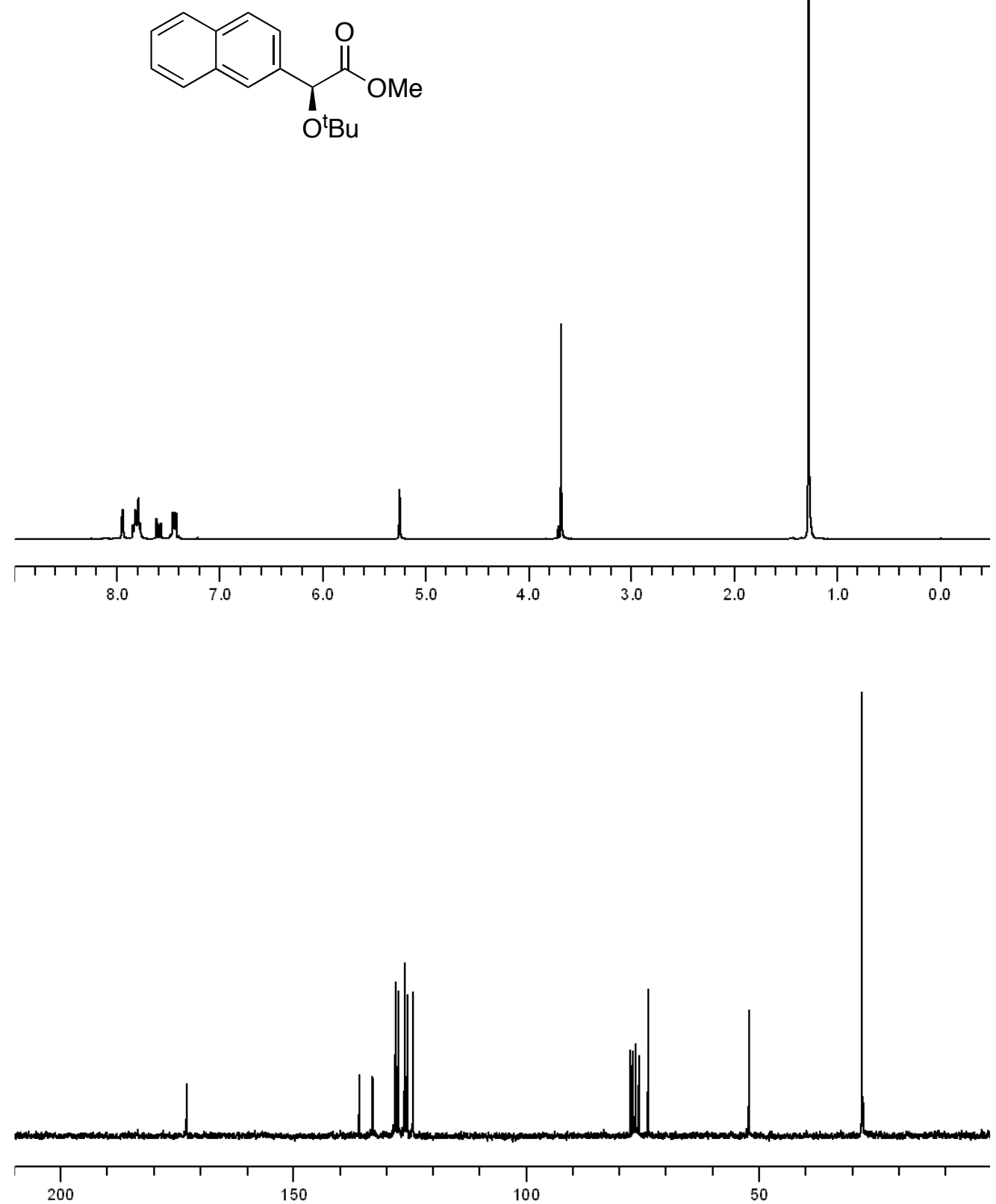

Figure 4S. ${ }^{1} \mathrm{H}$ NMR and ${ }^{13} \mathrm{C}$ NMR spectra of $(S)-17 \mathrm{a}$. 

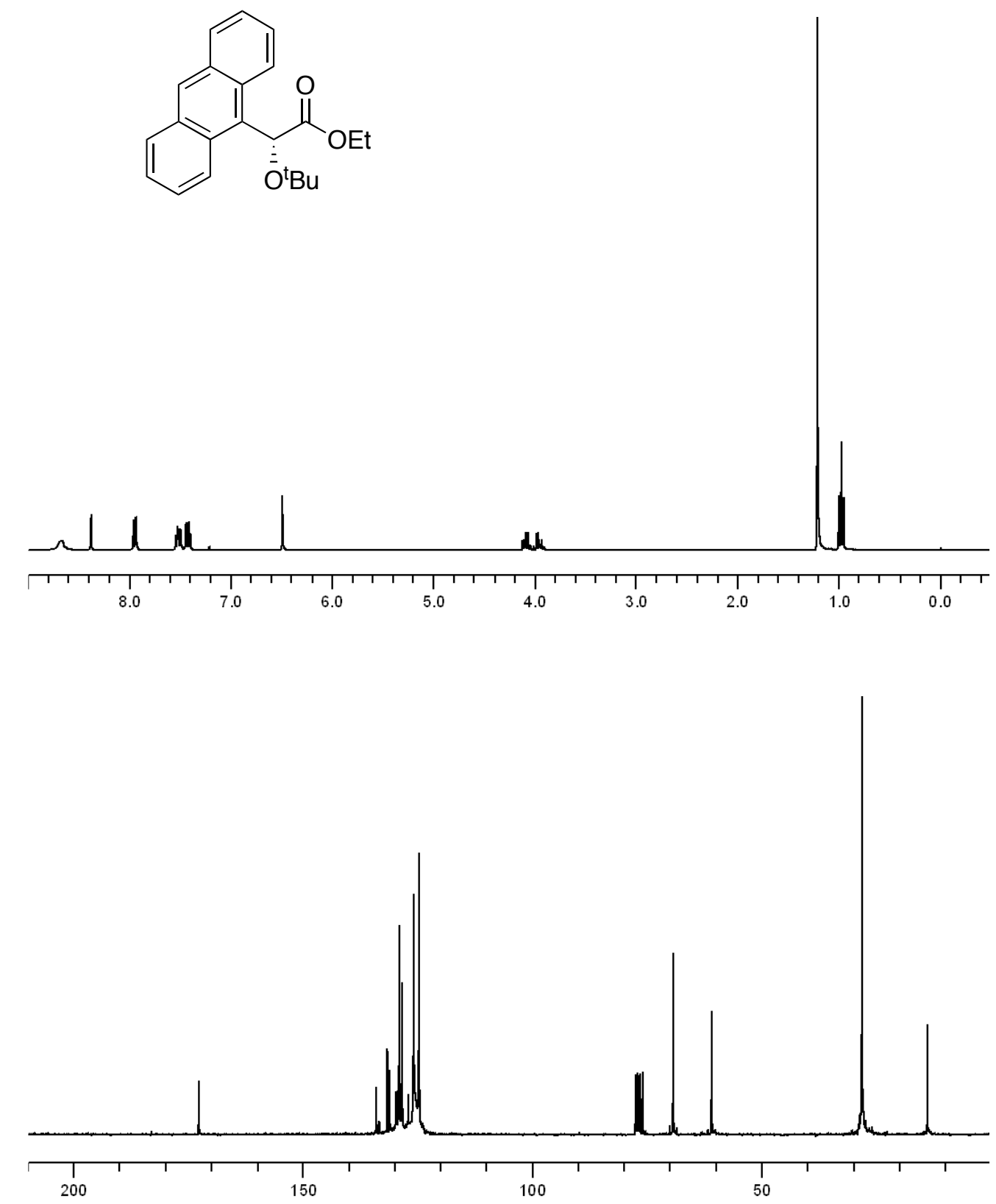

Figure 5S. ${ }^{1} \mathrm{H}$ NMR and ${ }^{13} \mathrm{C}$ NMR spectra of $(R)-18$ a. 
<smiles>CCCCC(C(=O)O)c1ccccc1</smiles>
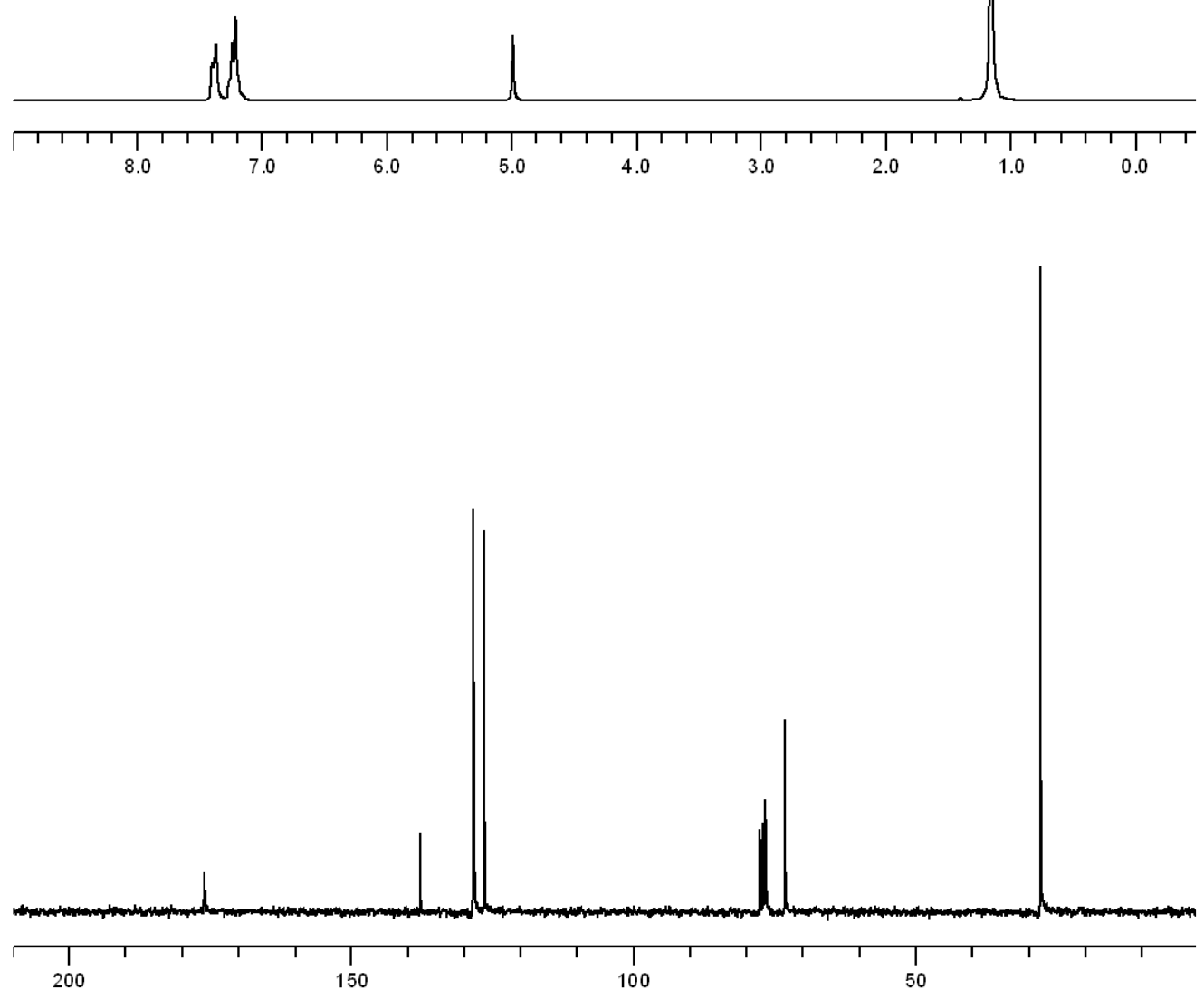

Figure 6S. ${ }^{1} \mathrm{H}$ NMR and ${ }^{13} \mathrm{C}$ NMR spectra of $(R)-16$. 

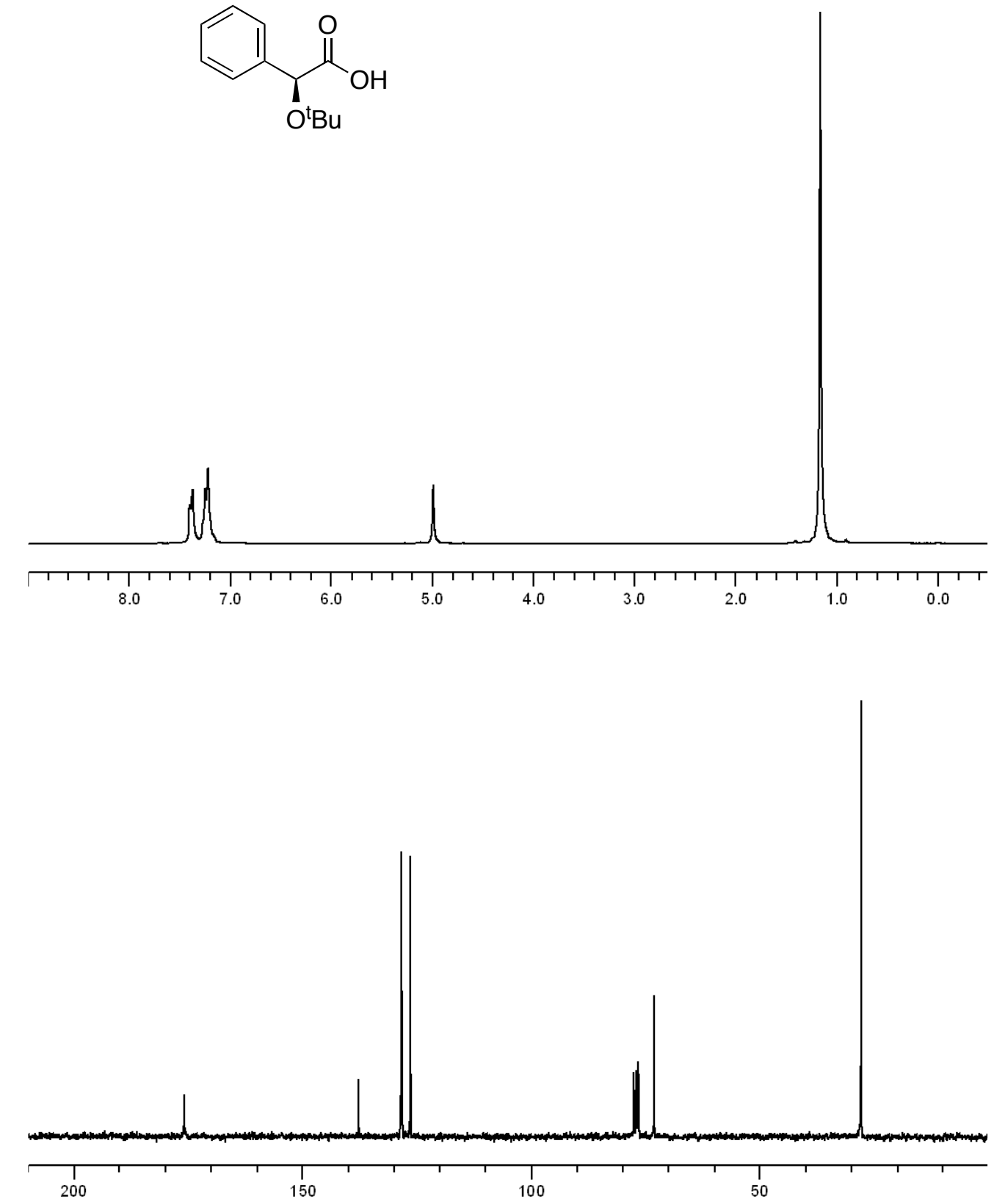

Figure 7S. ${ }^{1} \mathrm{H}$ NMR and ${ }^{13} \mathrm{C}$ NMR spectra of $(S)-16$. 
<smiles>CCCC[C@@H](C(=O)O)c1ccc2ccccc2c1</smiles>
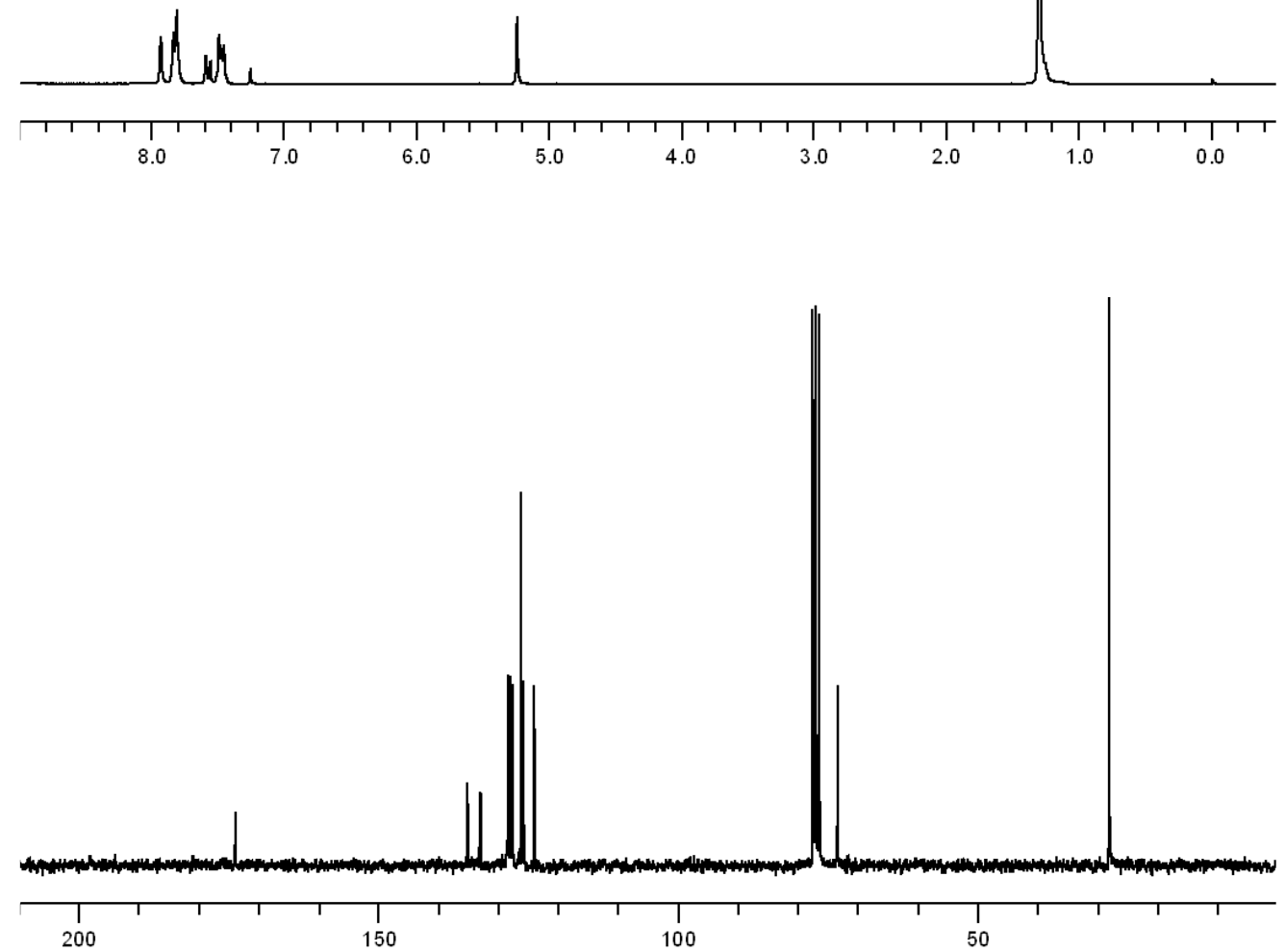

Figure 8S. ${ }^{1} \mathrm{H} \mathrm{NMR}$ and ${ }^{13} \mathrm{C}$ NMR spectra of $(R)-17$. 


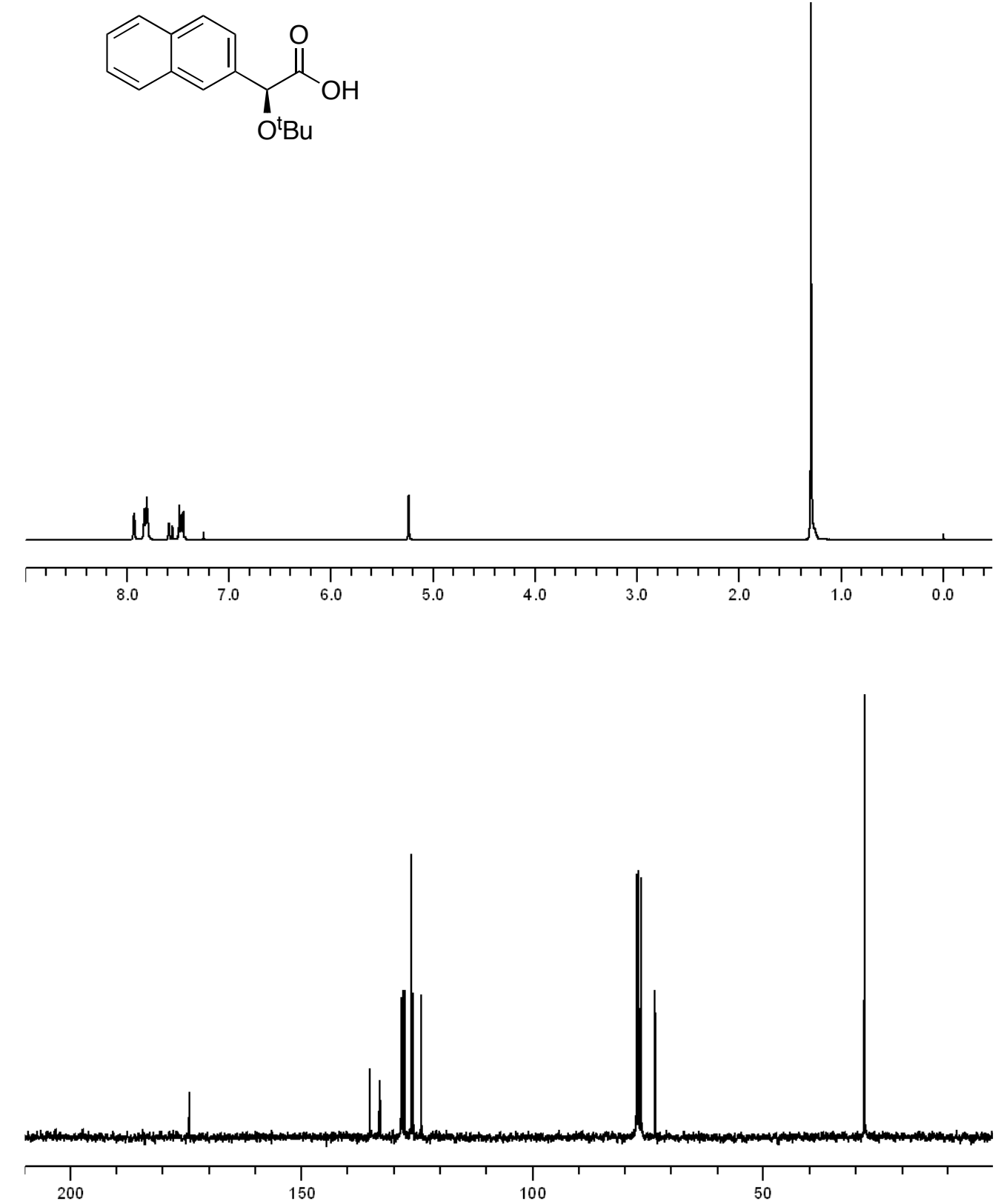

Figure 9S. ${ }^{1} \mathrm{H}$ NMR and ${ }^{13} \mathrm{C}$ NMR spectra of $(S)-17$. 
<smiles>CCCC(C(=O)O)c1c2ccccc2cc2ccccc12</smiles>
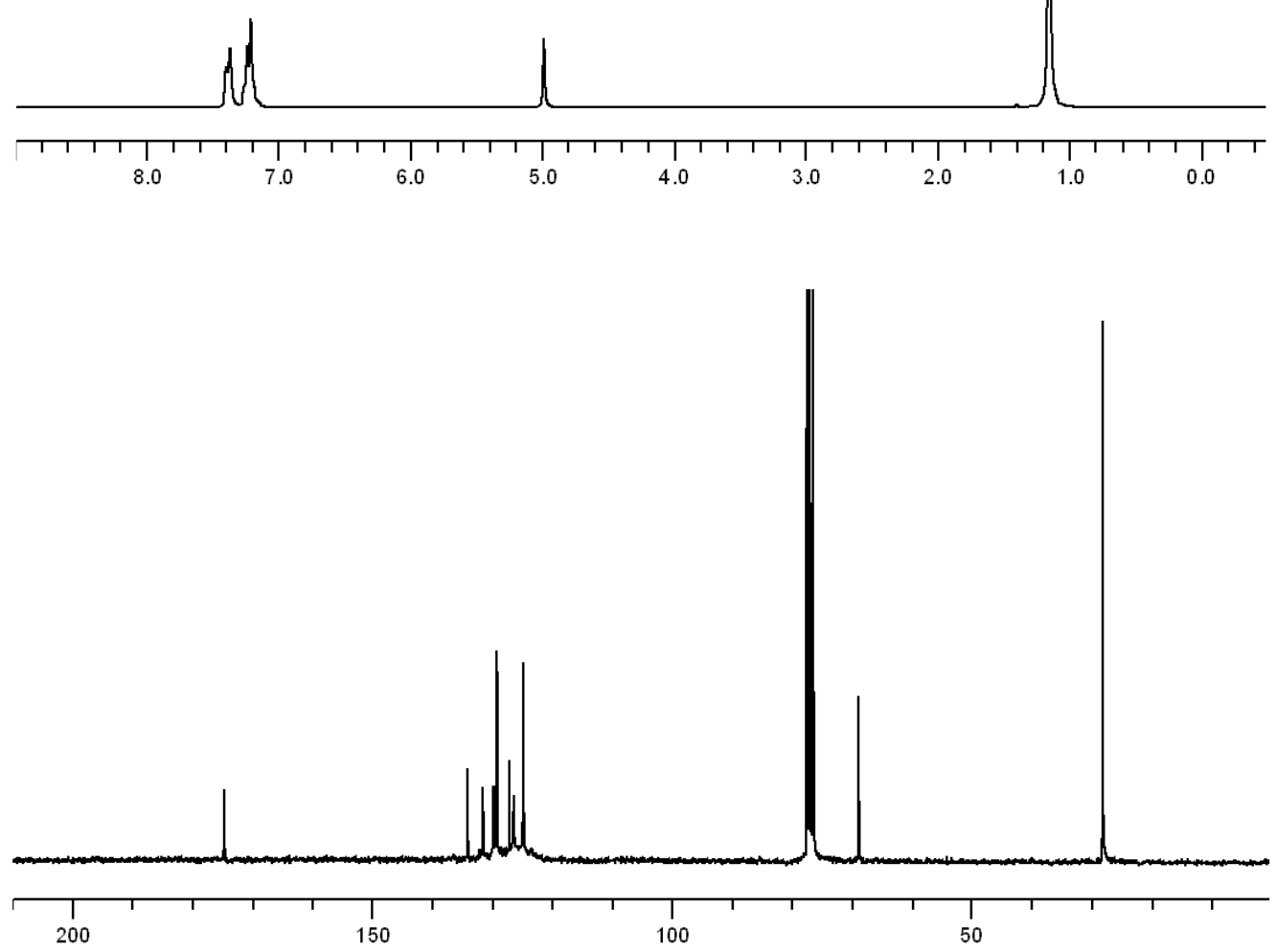

Figure 10S. ${ }^{1} \mathrm{H}$ NMR and ${ }^{13} \mathrm{C}$ NMR spectra of $(R)-18$. 

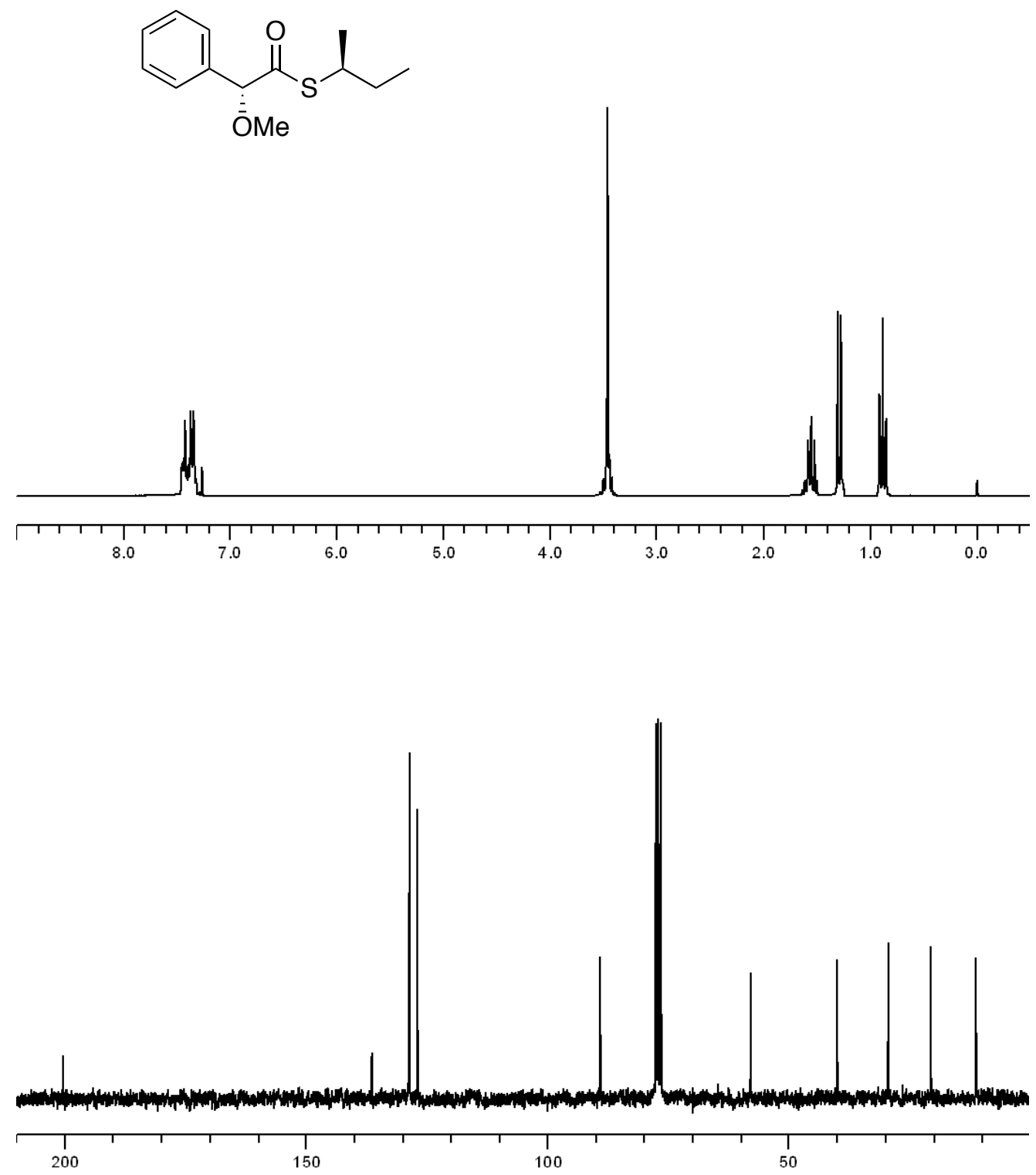

Figure 11S. ${ }^{1} \mathrm{H}$ NMR and ${ }^{13} \mathrm{C}$ NMR spectra of $(R)$-MPA-5. 

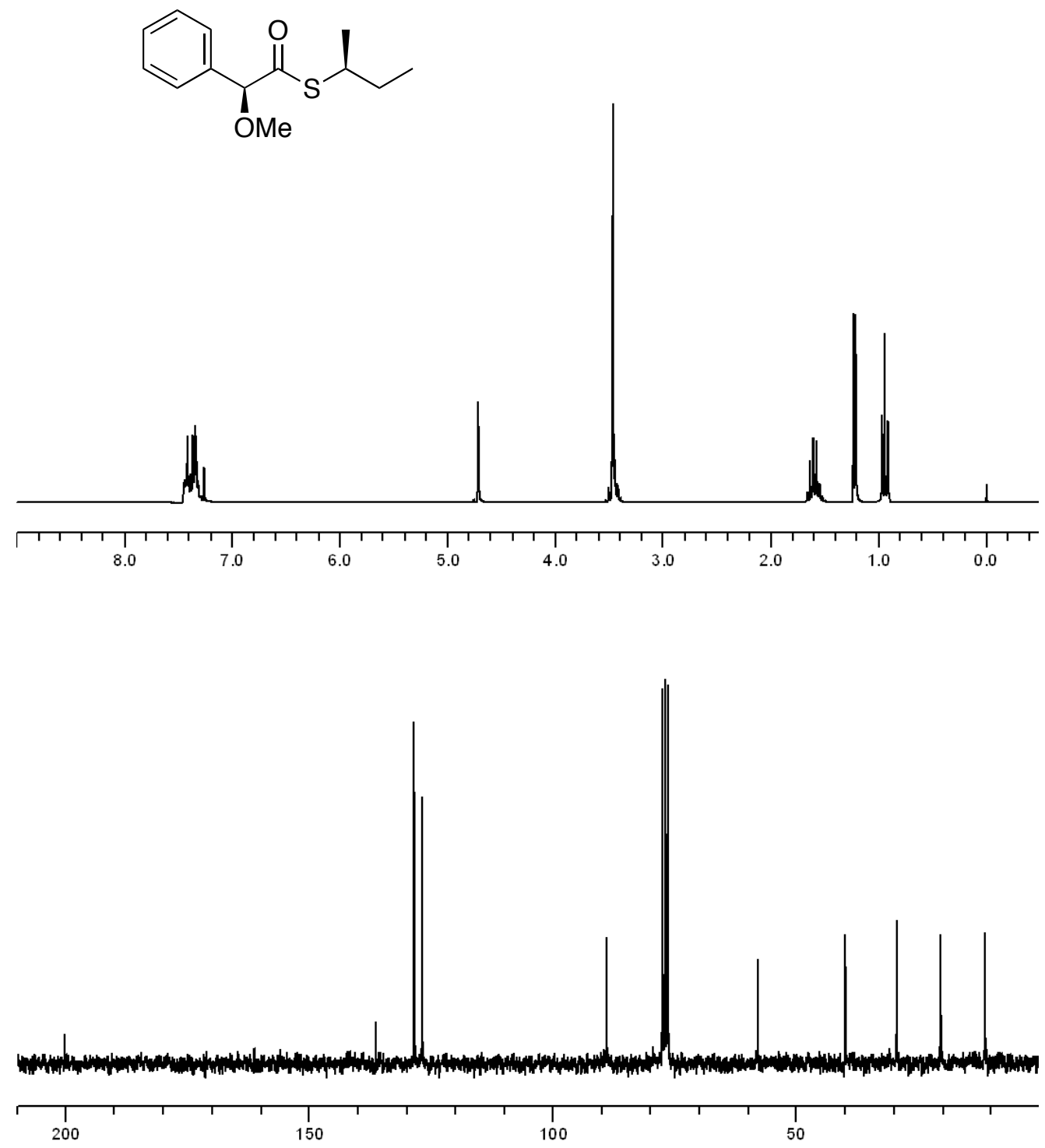

Figure 12S. ${ }^{1} \mathrm{H}$ NMR and ${ }^{13} \mathrm{C}$ NMR spectra of $(S)$-MPA-5. 
<smiles>CCCCCC[C@H](C)SC(=O)[C@H](OC)c1ccccc1</smiles>
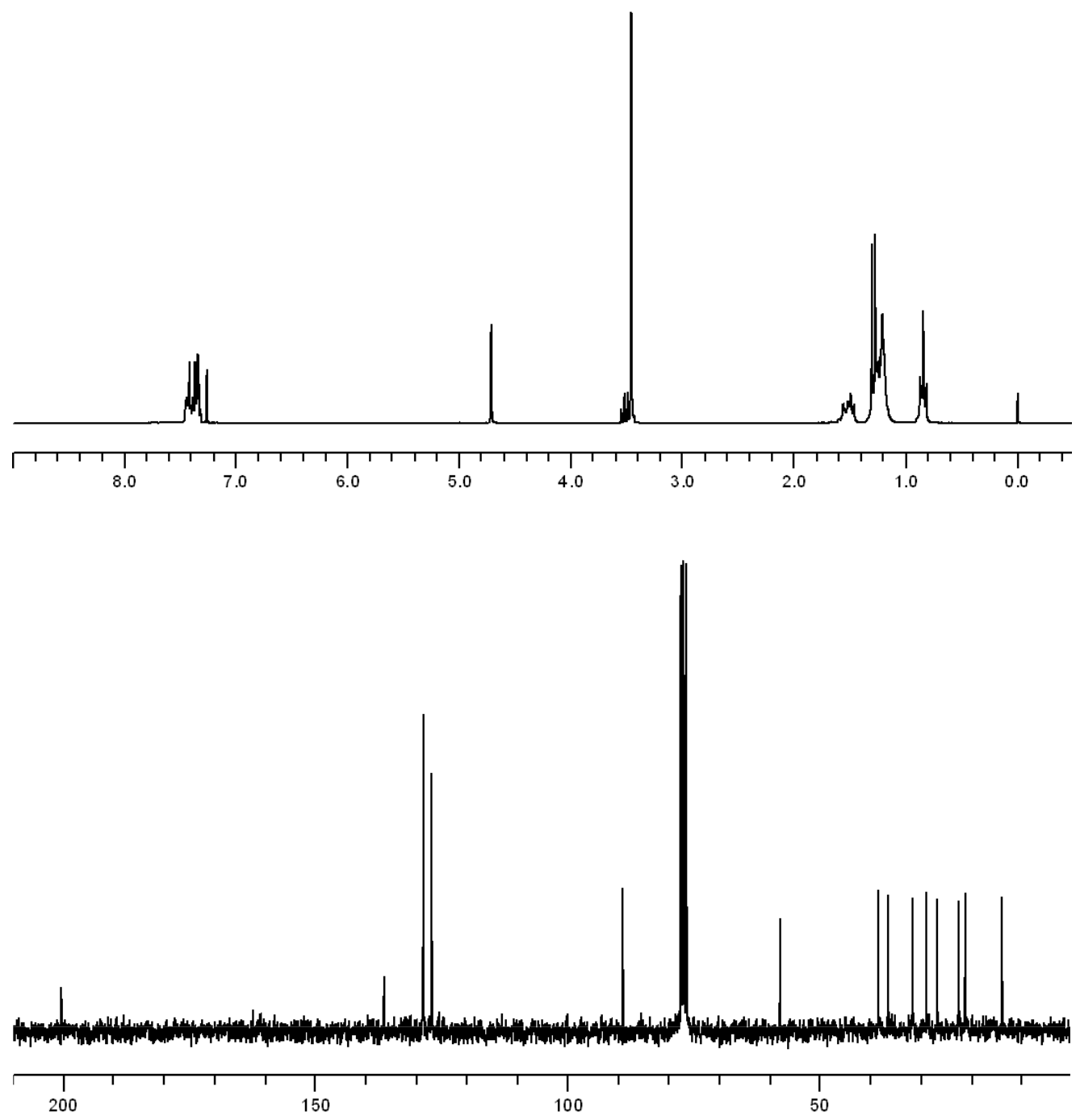

Figure 13S. ${ }^{1} \mathrm{H}$ NMR and ${ }^{13} \mathrm{C}$ NMR spectra of (R)-MPA-6.

S33 

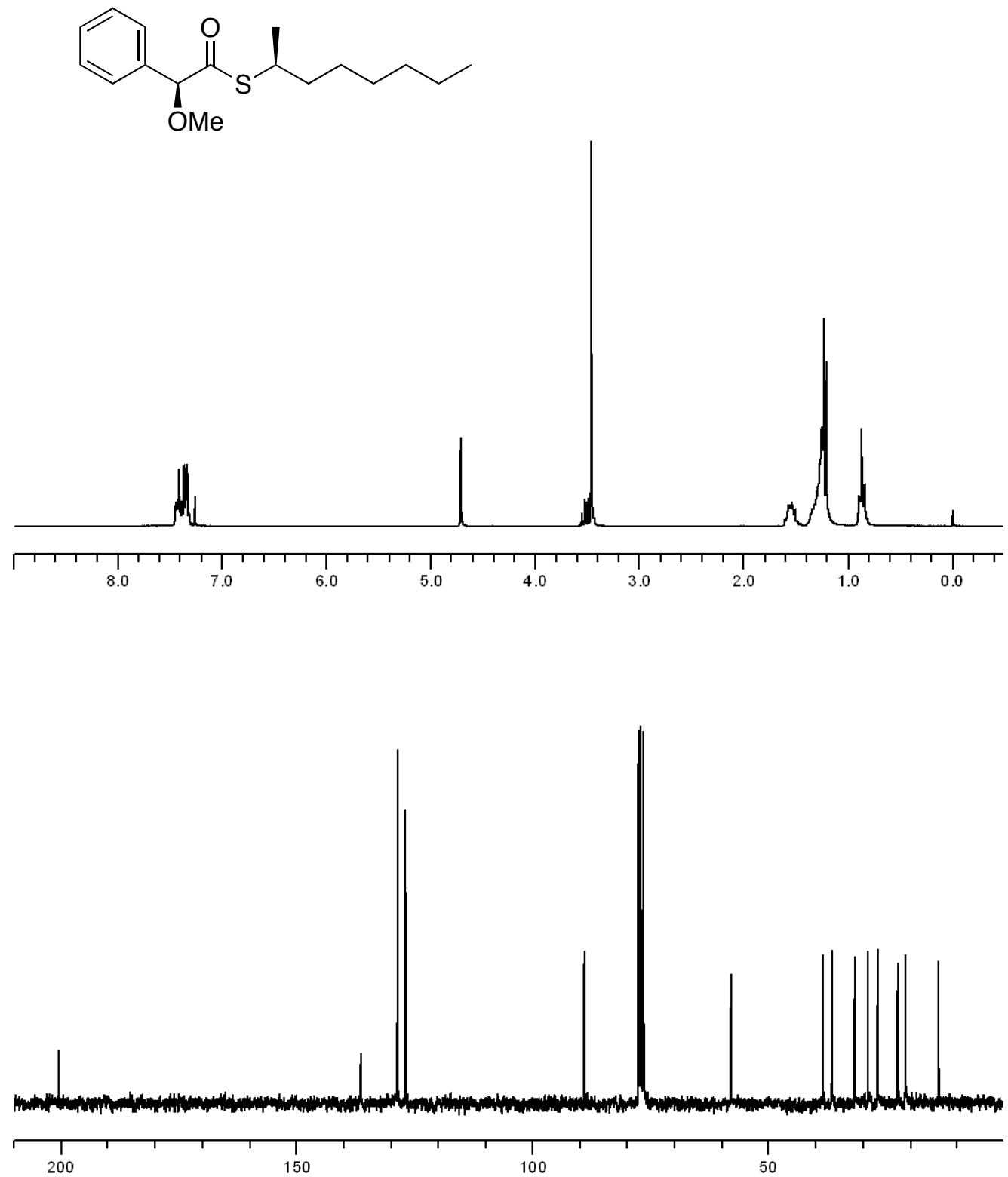

Figure 14S. ${ }^{1} \mathrm{H}$ NMR and ${ }^{13} \mathrm{C}$ NMR spectra of $(S)$-MPA-6. 

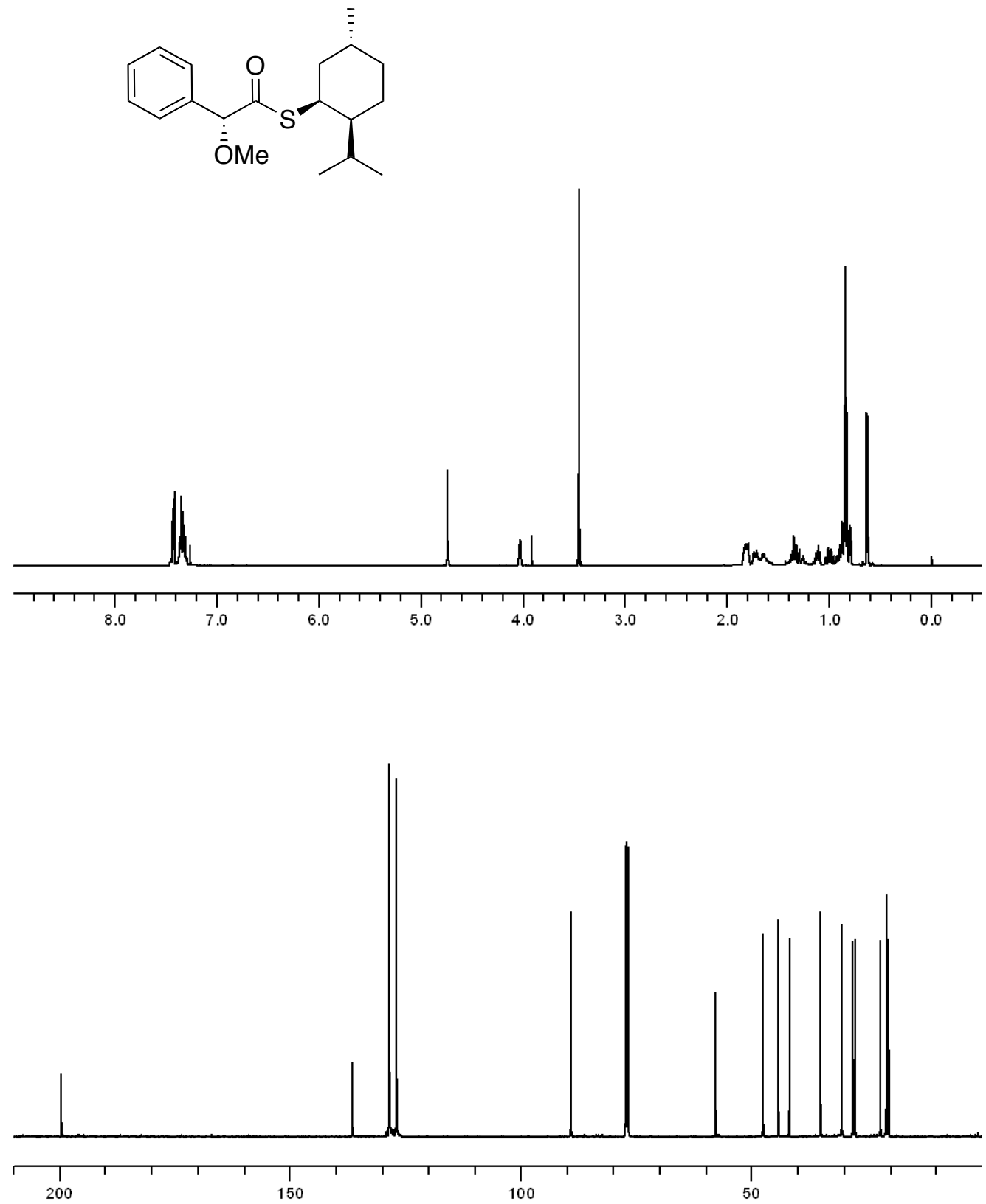

Figure 15S. ${ }^{1} \mathrm{H}$ NMR and ${ }^{13} \mathrm{C}$ NMR spectra of $(R)$-MPA-7. 

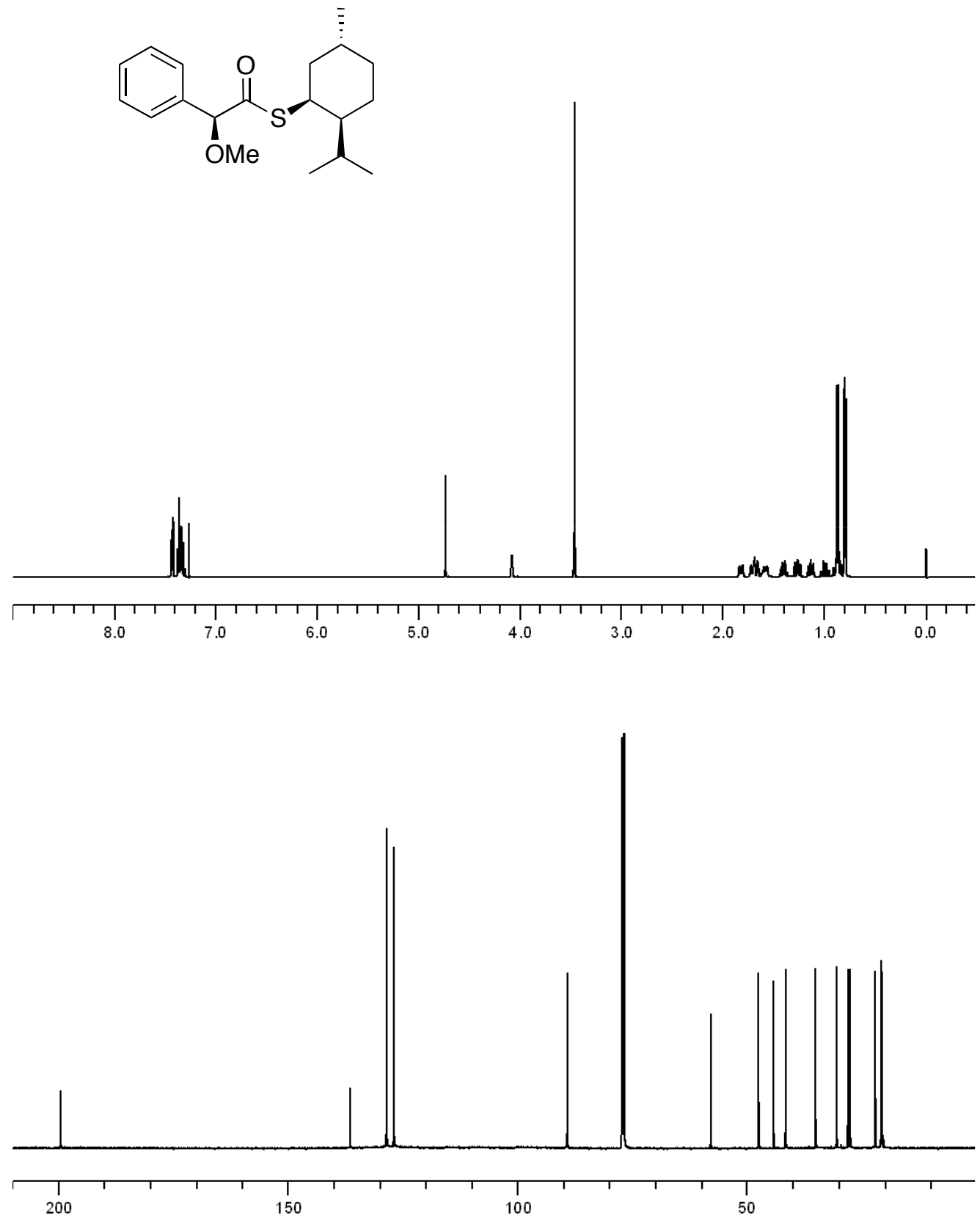

Figure 16S. ${ }^{1} \mathrm{H}$ NMR and ${ }^{13} \mathrm{C}$ NMR spectra of $(S)$-MPA-7. 

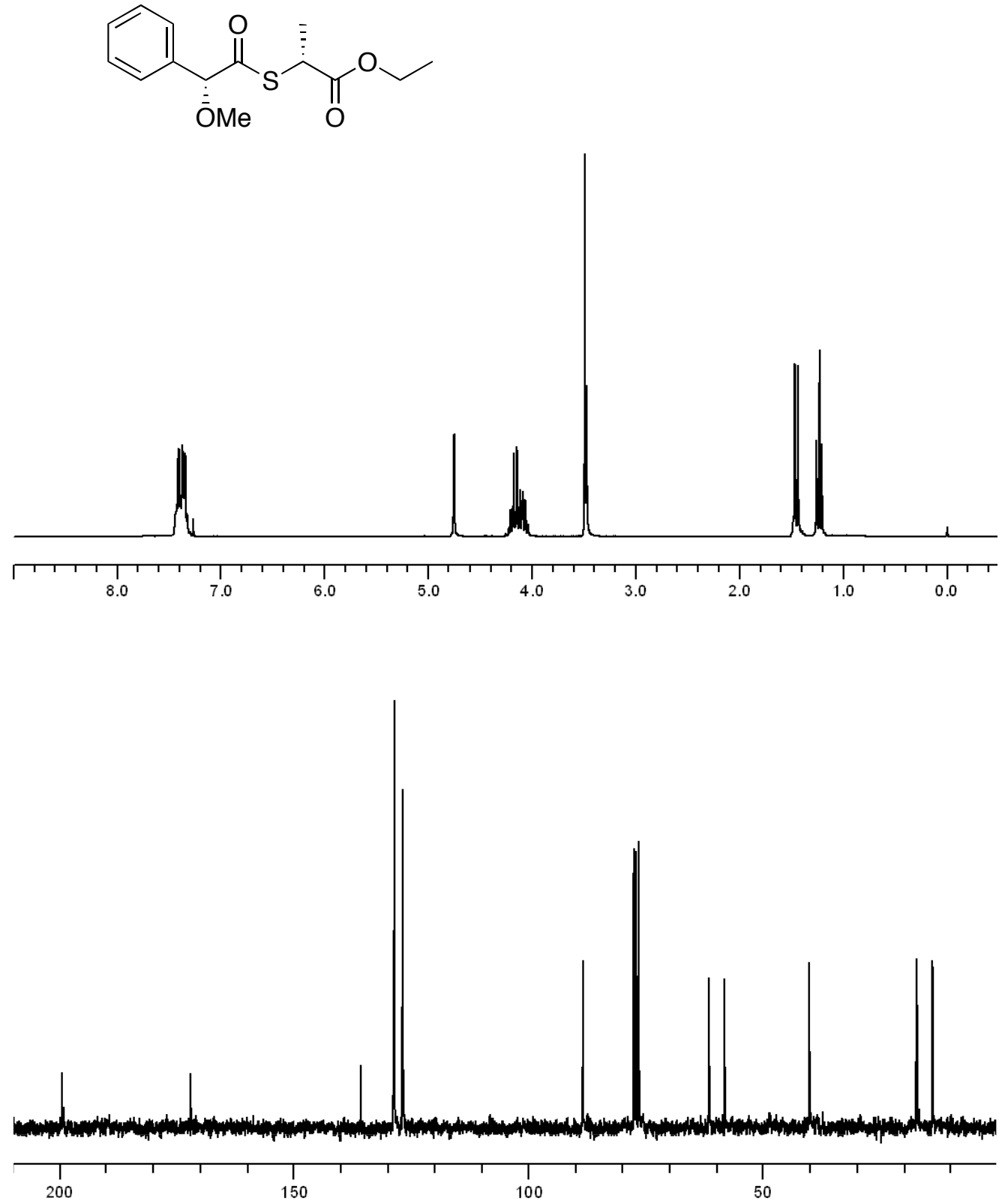

Figure 17S. ${ }^{1} \mathrm{H}$ NMR and ${ }^{13} \mathrm{C}$ NMR spectra of $(R)$-MPA-8. 


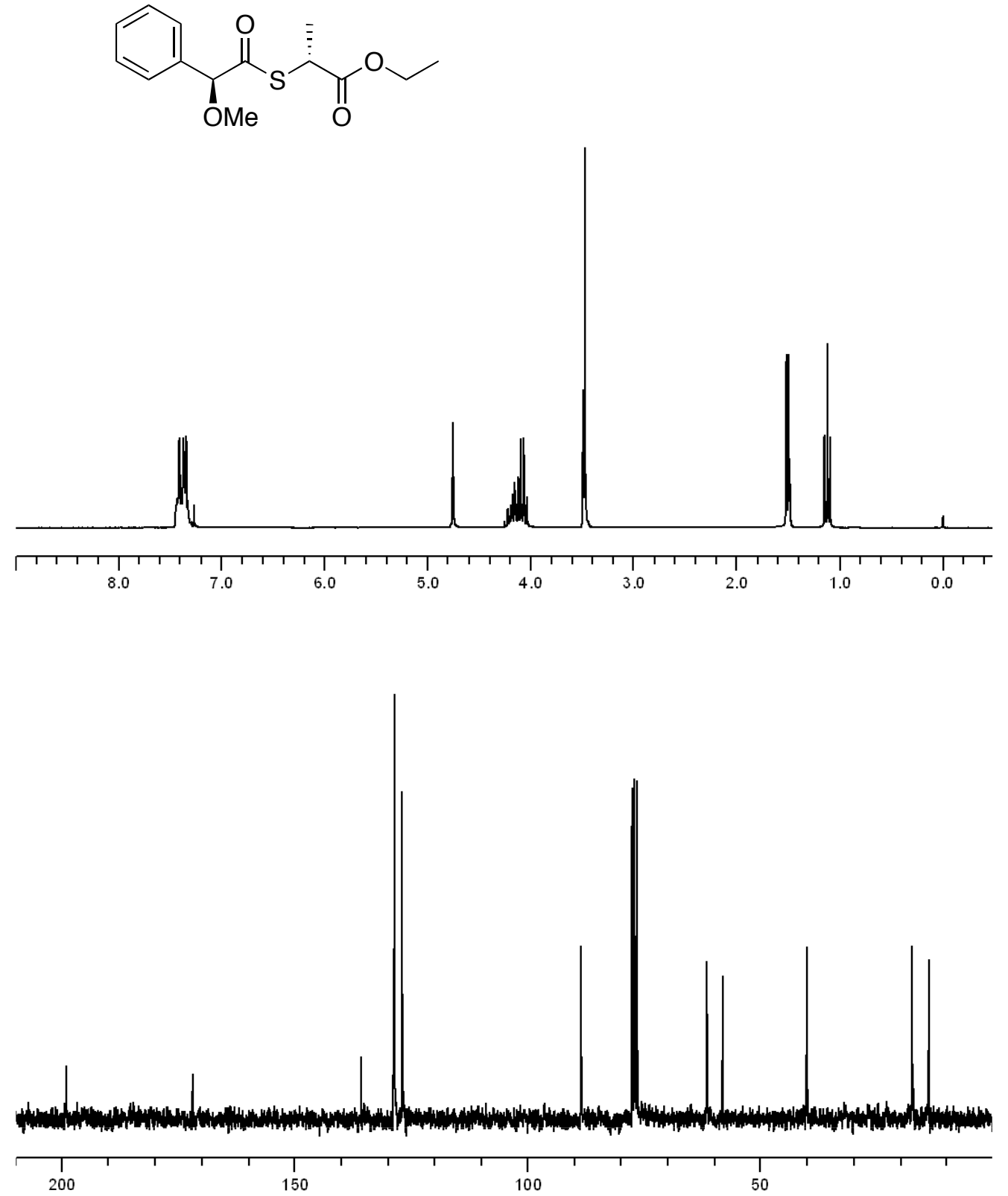

Figure 18S. ${ }^{1} \mathrm{H}$ NMR and ${ }^{13} \mathrm{C}$ NMR spectra of $(S)$-MPA-8. 


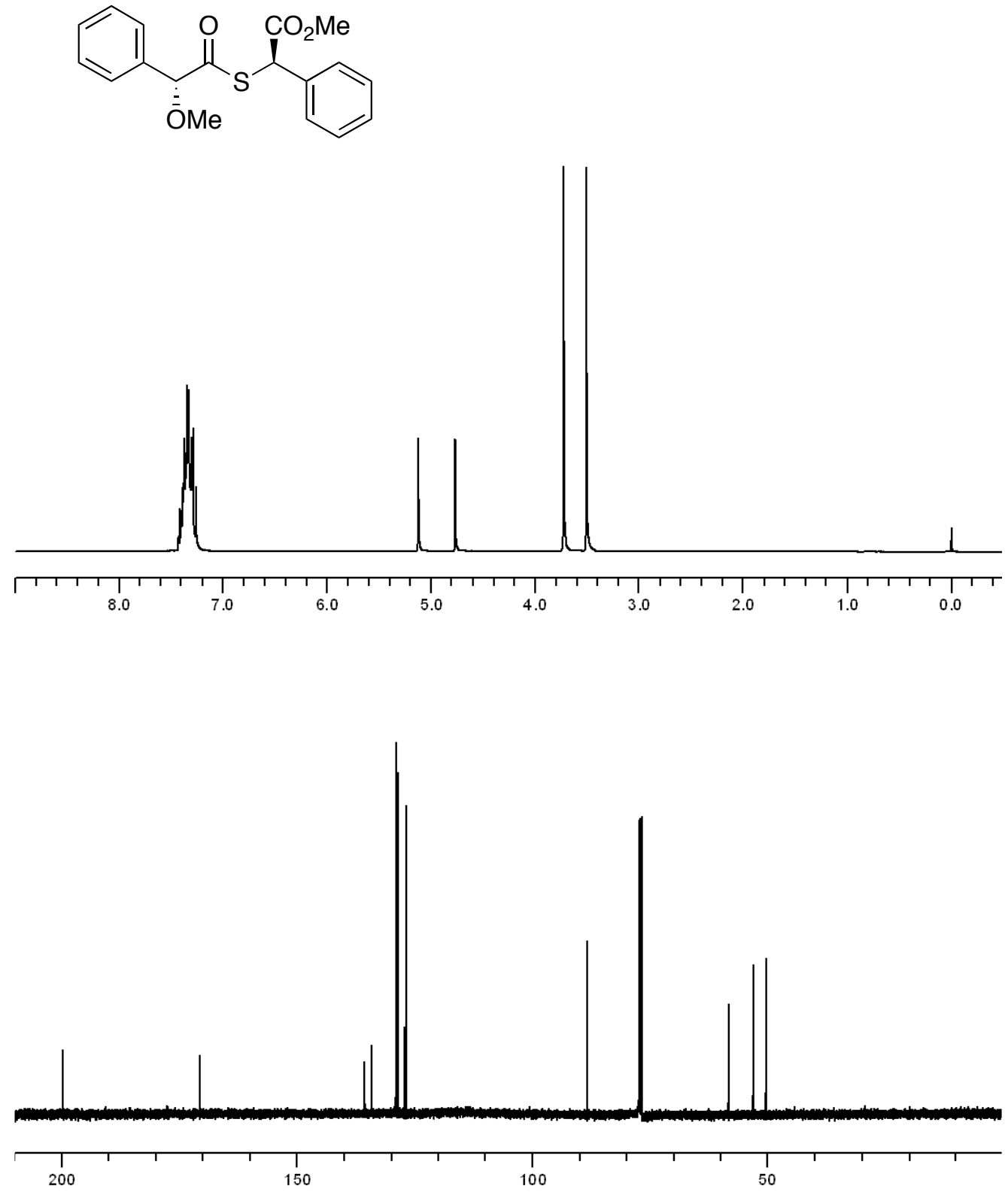

Figure 19S. ${ }^{1} \mathrm{H}$ NMR and ${ }^{13} \mathrm{C}$ NMR spectra of $(R)$-MPA-9. 

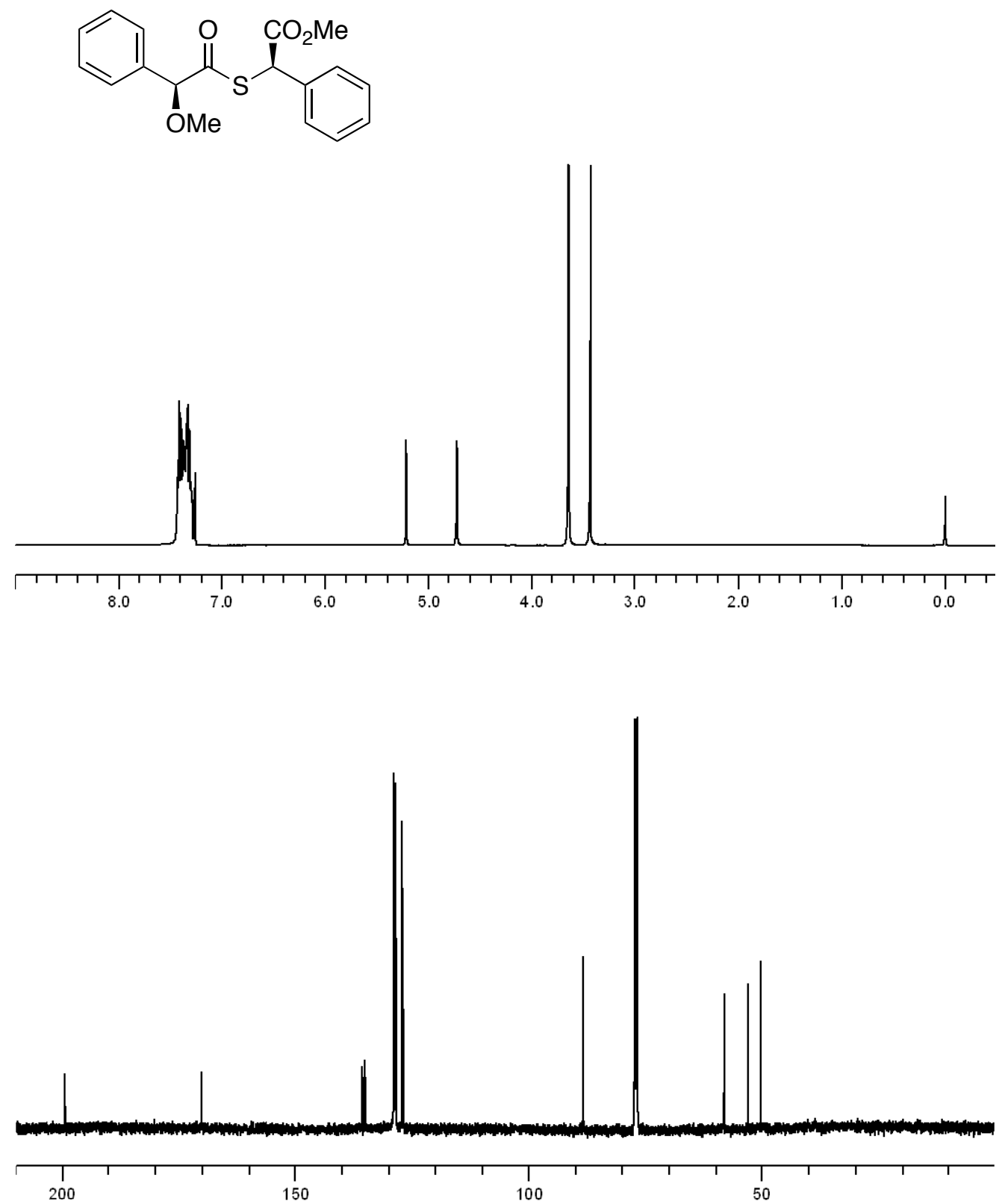

Figure 20S. ${ }^{1} \mathrm{H}$ NMR and ${ }^{13} \mathrm{C}$ NMR spectra of $(S)$-MPA-9. 

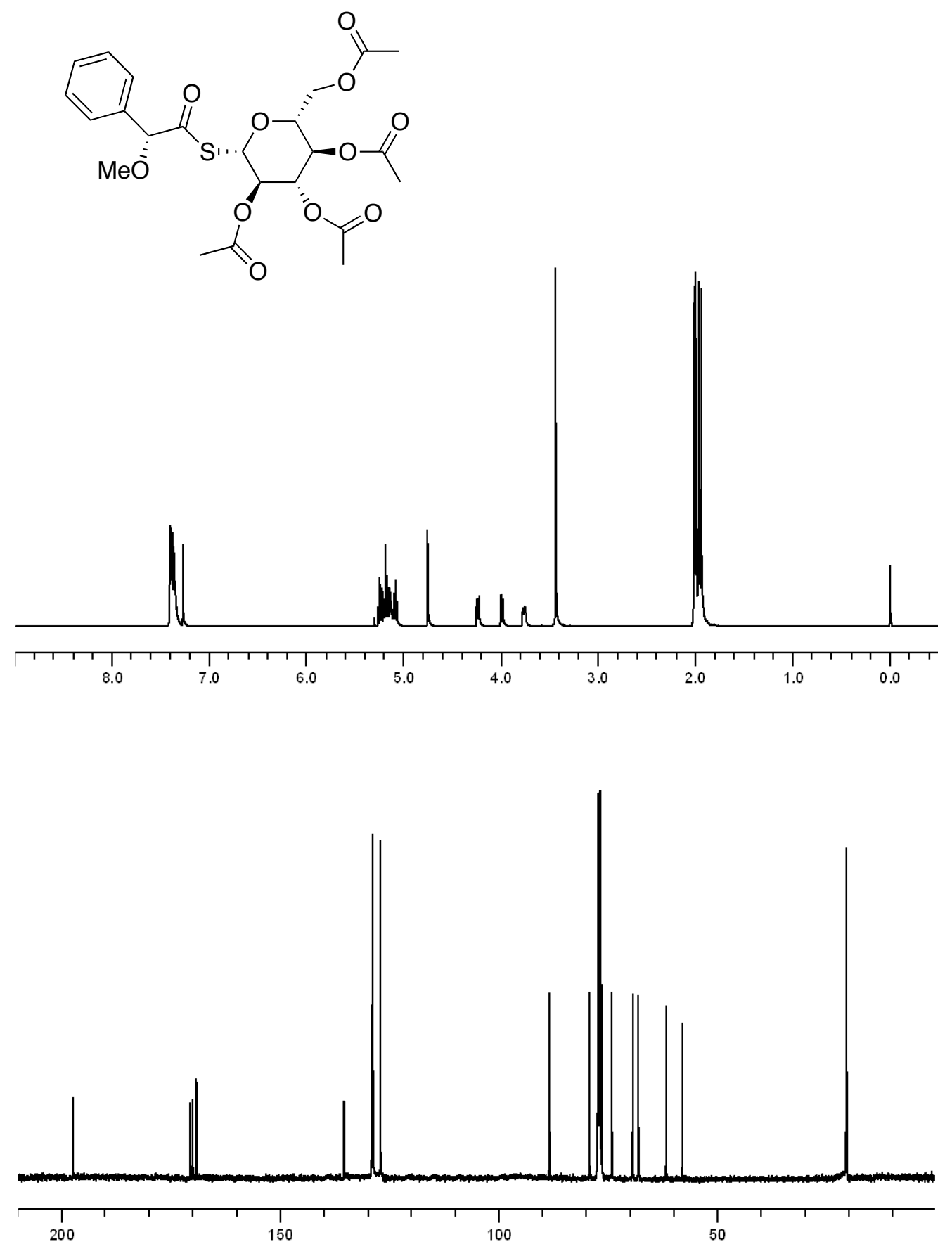

Figure 21S. ${ }^{1} \mathrm{H}$ NMR and ${ }^{13} \mathrm{C}$ NMR spectra of $(R)$-MPA-10. 

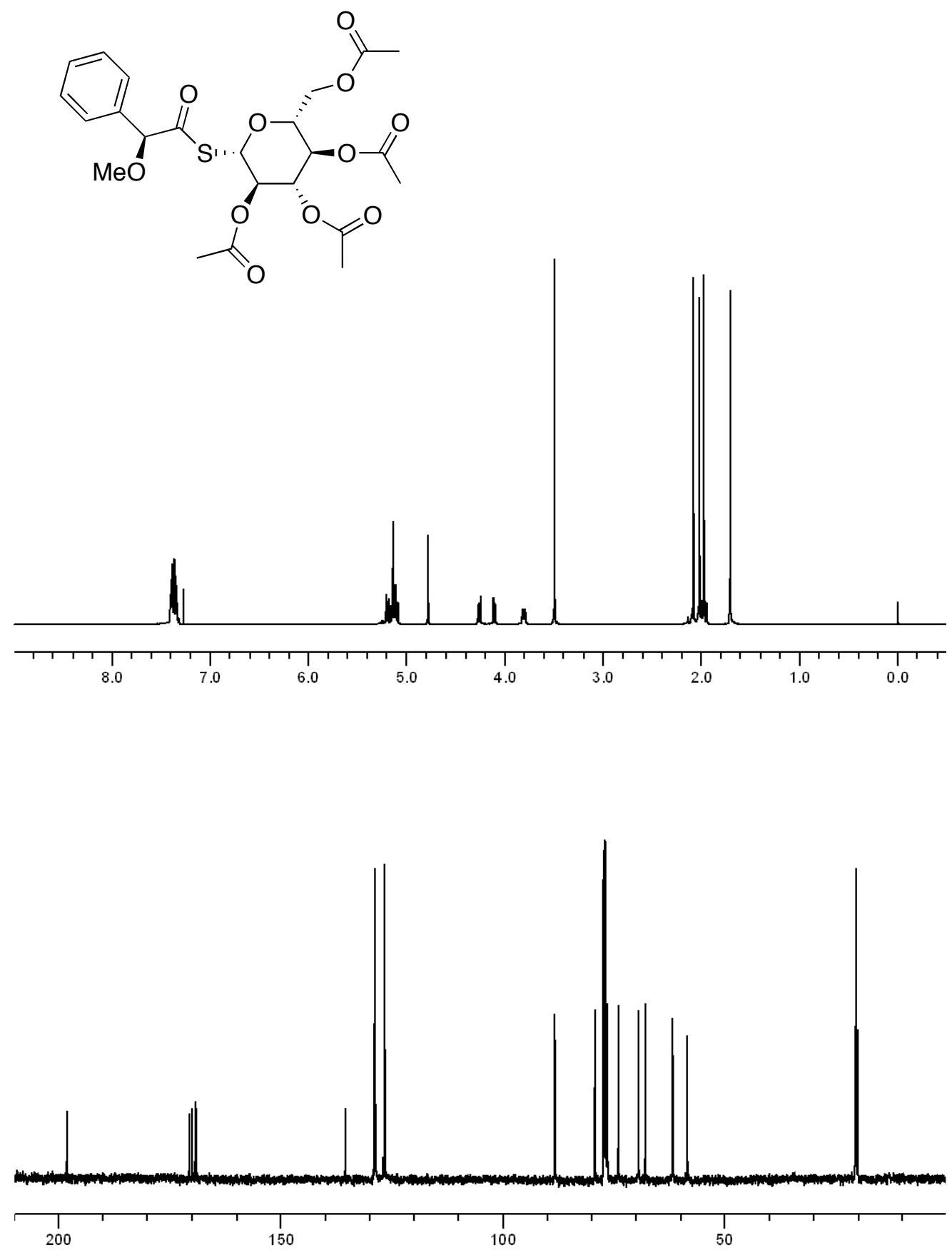

Figure 22S. ${ }^{1} \mathrm{H}$ NMR and ${ }^{13} \mathrm{C}$ NMR spectra of $(S)$-MPA-10. 

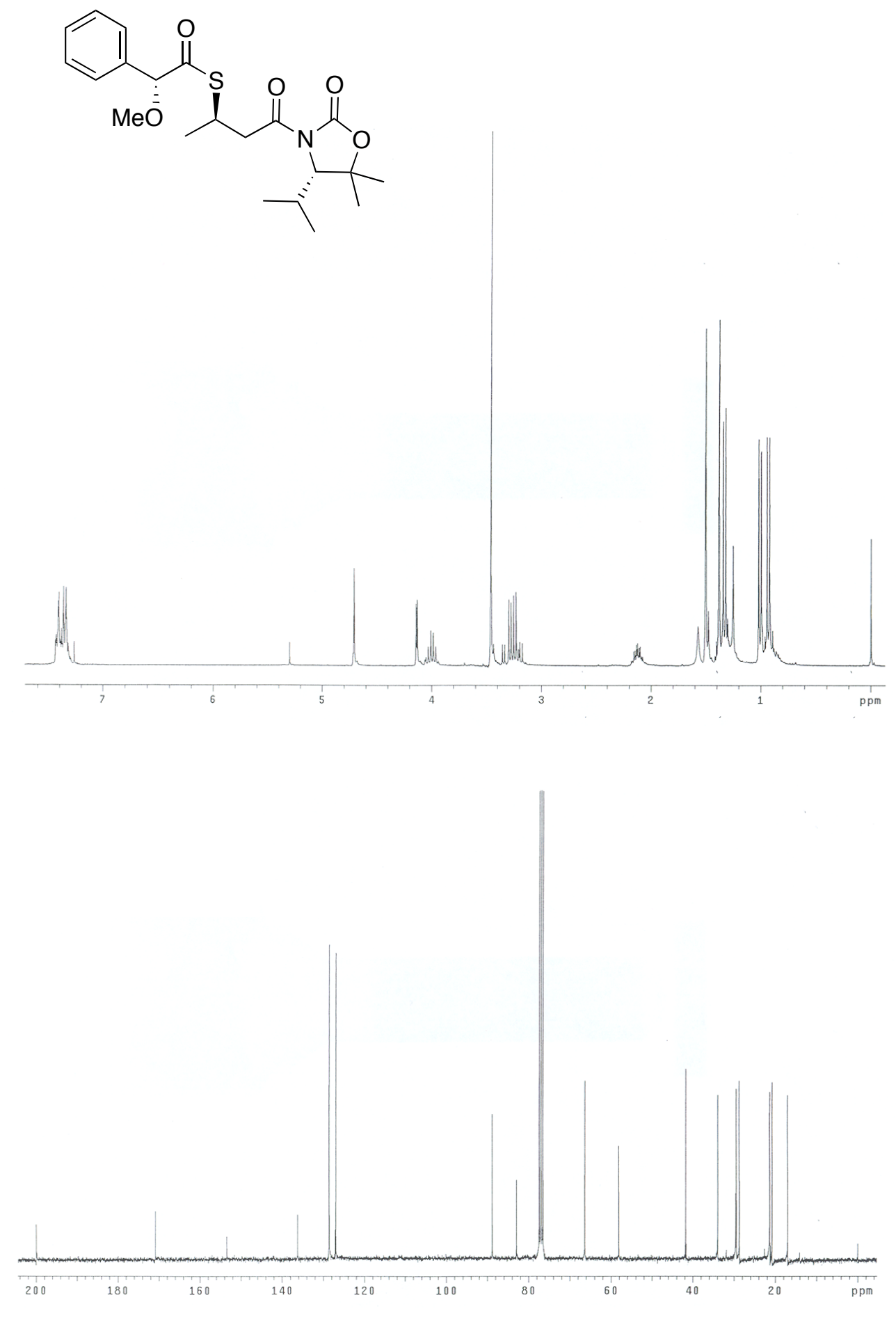

Figure 23S. ${ }^{1} \mathrm{H}$ NMR and ${ }^{13} \mathrm{C}$ NMR spectra of $(R)$-MPA-11. 

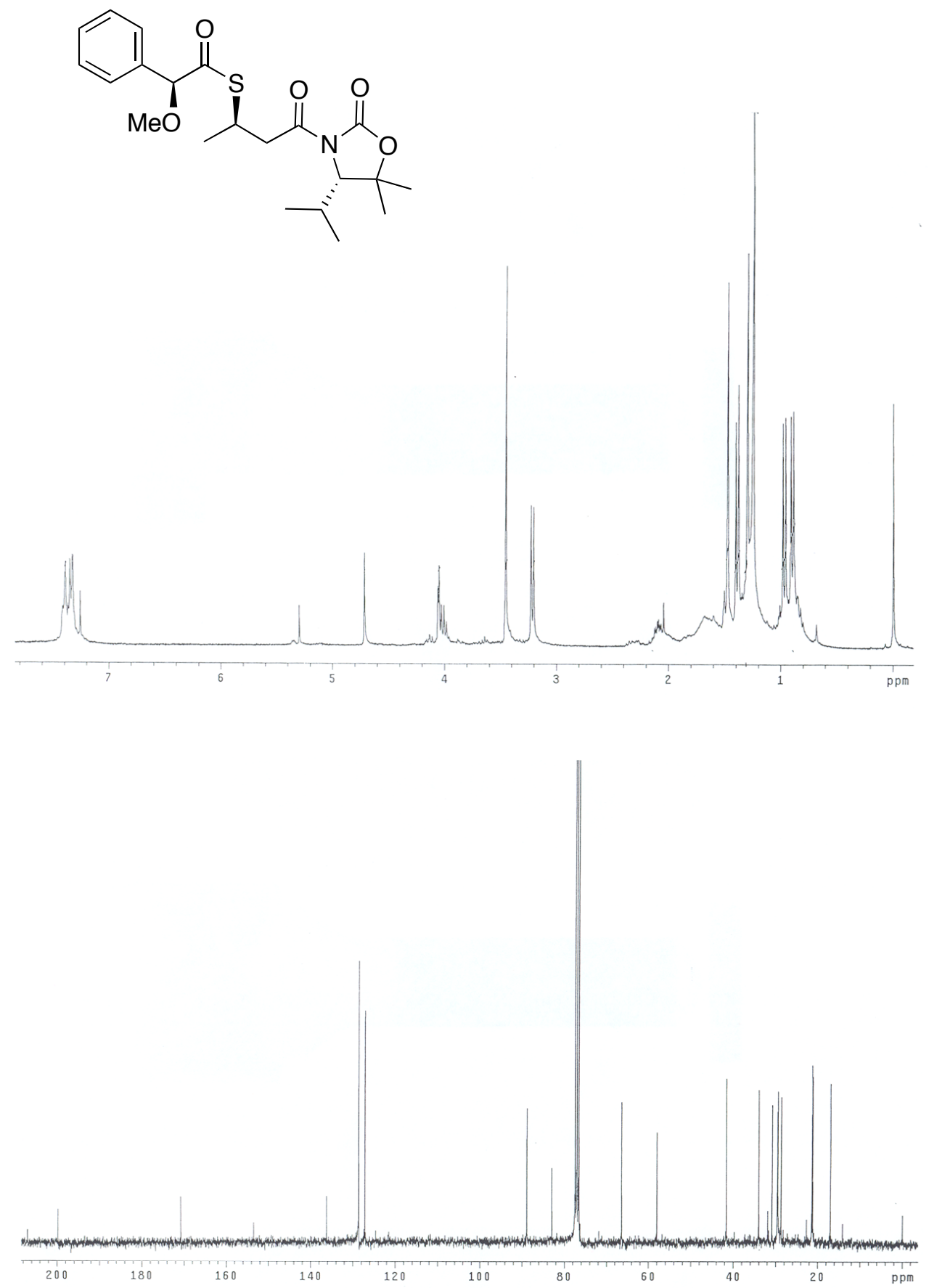

Figure 24S. ${ }^{1} \mathrm{H}$ NMR and ${ }^{13} \mathrm{C}$ NMR spectra of $(S)$-MPA-11. 

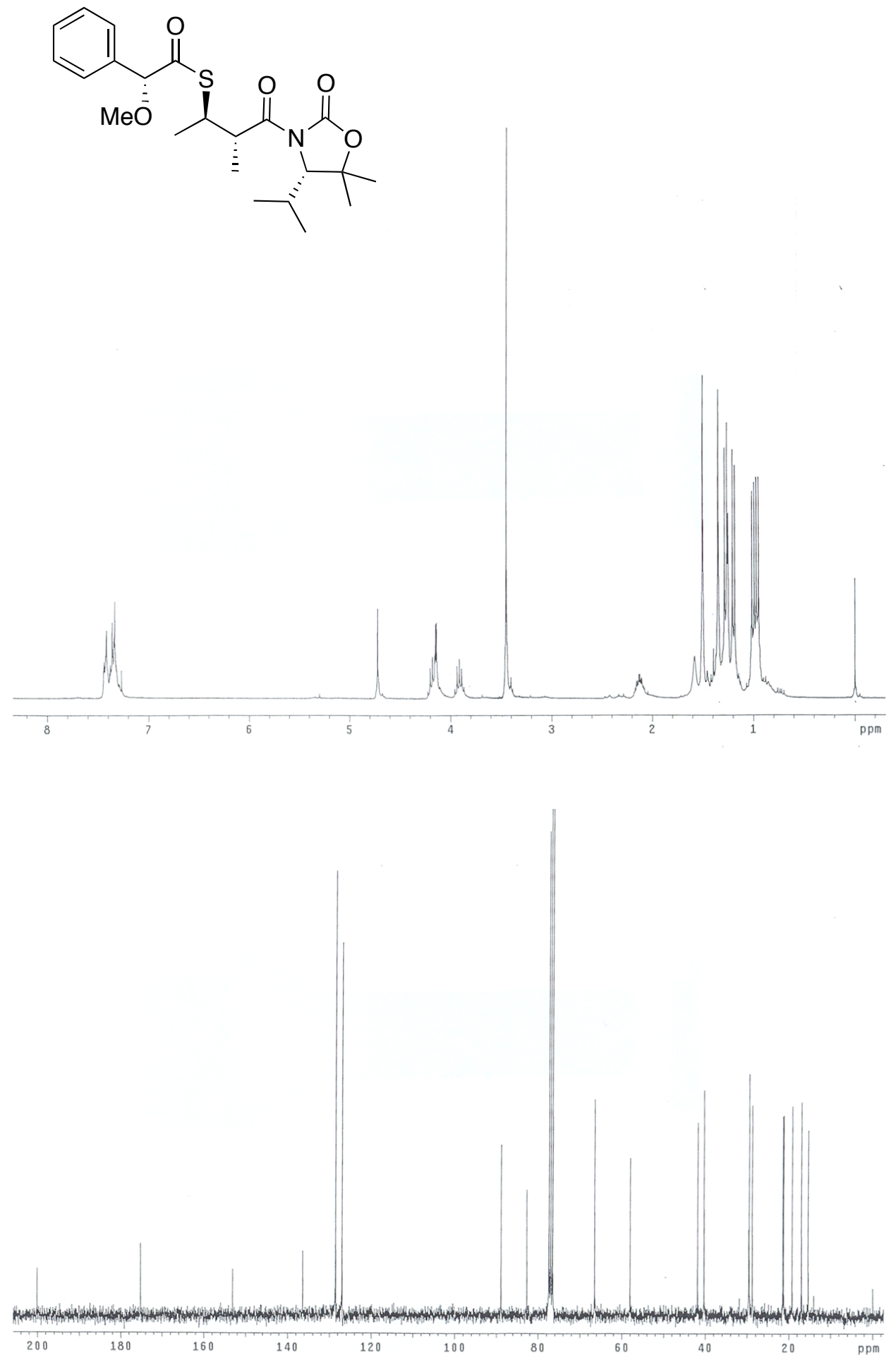

Figure 25S. ${ }^{1} \mathrm{H}$ NMR and ${ }^{13} \mathrm{C}$ NMR spectra of $(R)$-MPA-12. 

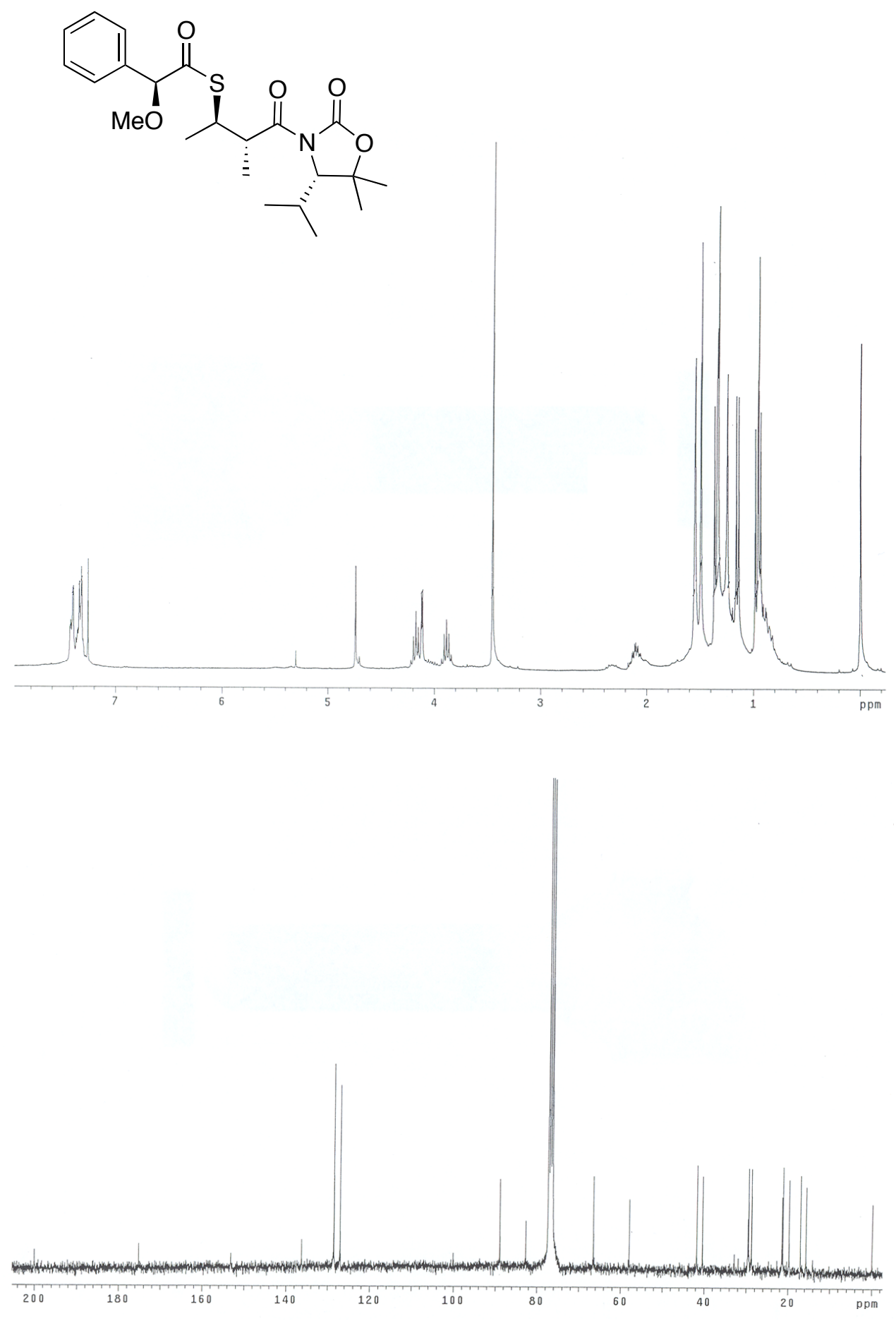

Figure 26S. ${ }^{1} \mathrm{H}$ NMR and ${ }^{13} \mathrm{C}$ NMR spectra of $(S)$-MPA-12. 

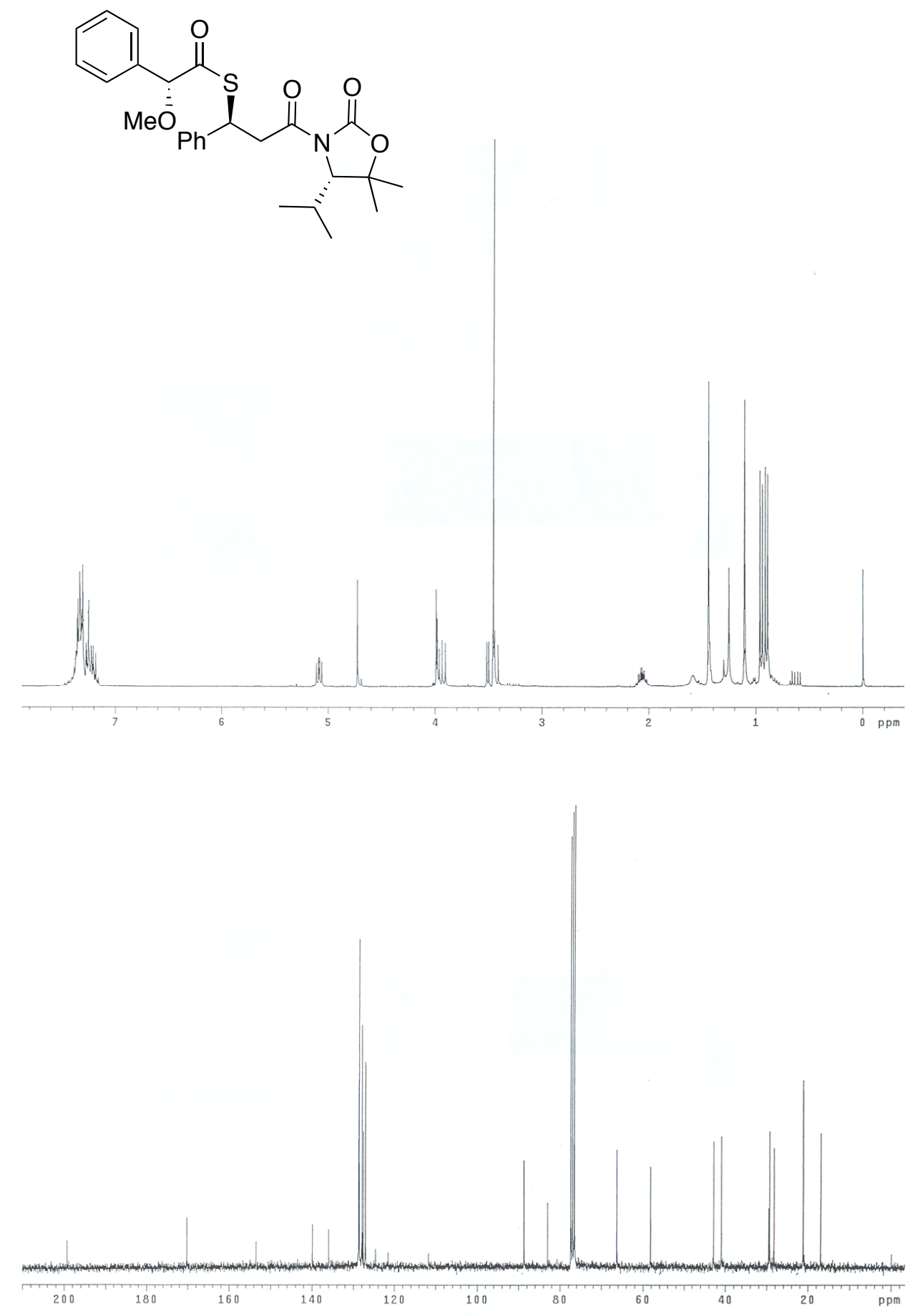

Figure 27S. ${ }^{1} \mathrm{H}$ NMR and ${ }^{13} \mathrm{C}$ NMR spectra of $(R)$-MPA-13. 

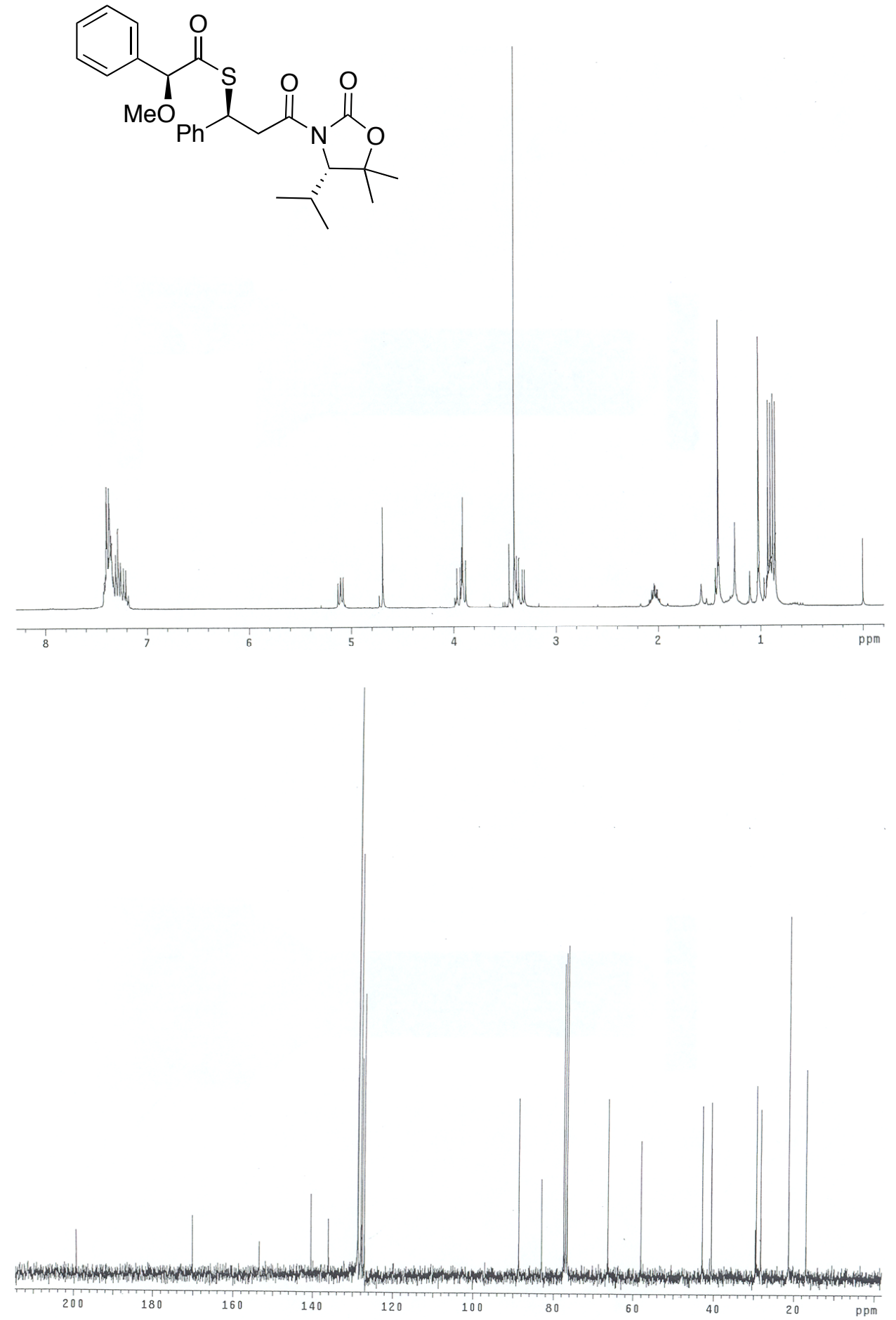

Figure 28S. ${ }^{1} \mathrm{H}$ NMR and ${ }^{13} \mathrm{C}$ NMR spectra of $(S)$-MPA-13. 

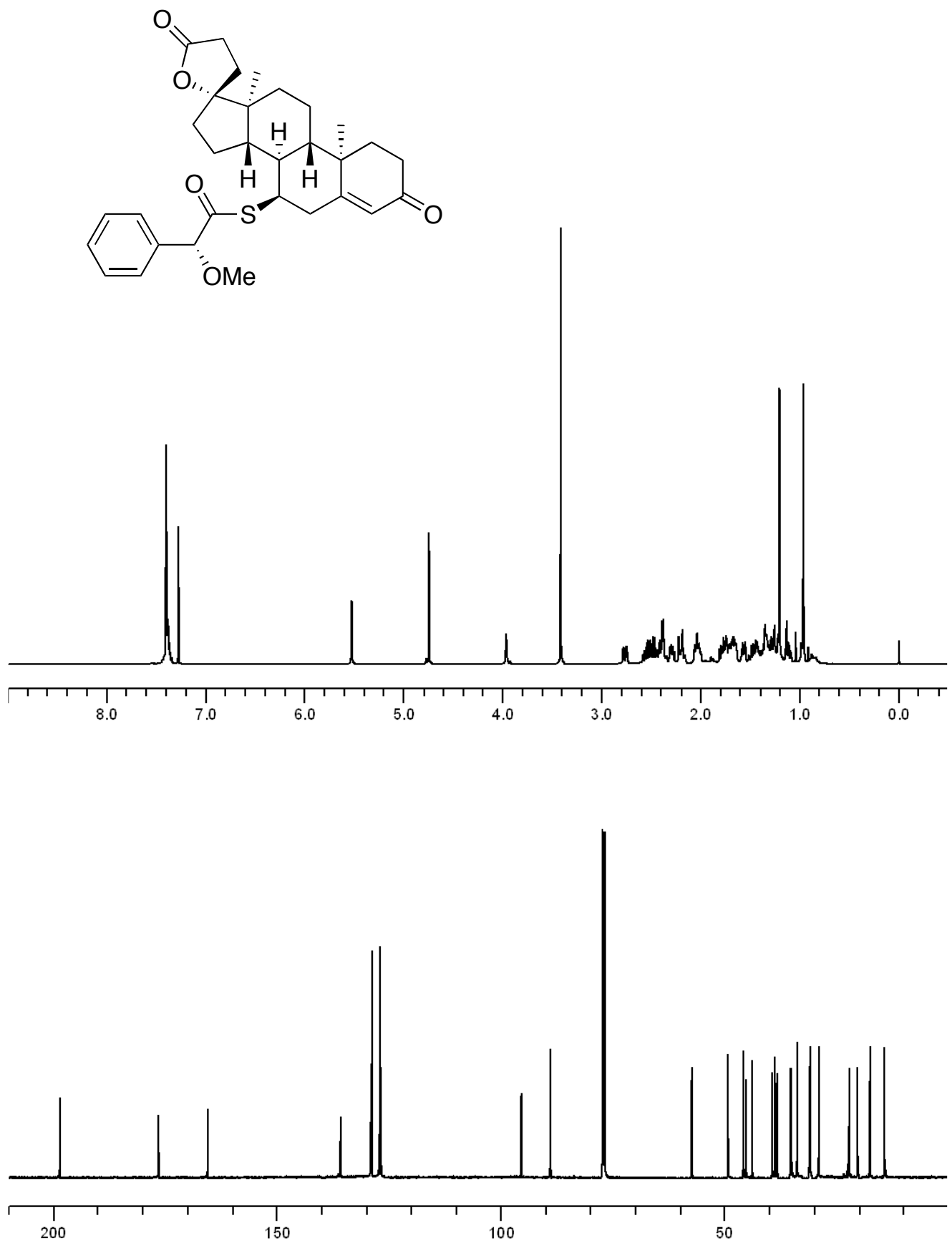

Figure 29S. ${ }^{1} \mathrm{H}$ NMR and ${ }^{13} \mathrm{C}$ NMR spectra of $(R)$-MPA-14. 

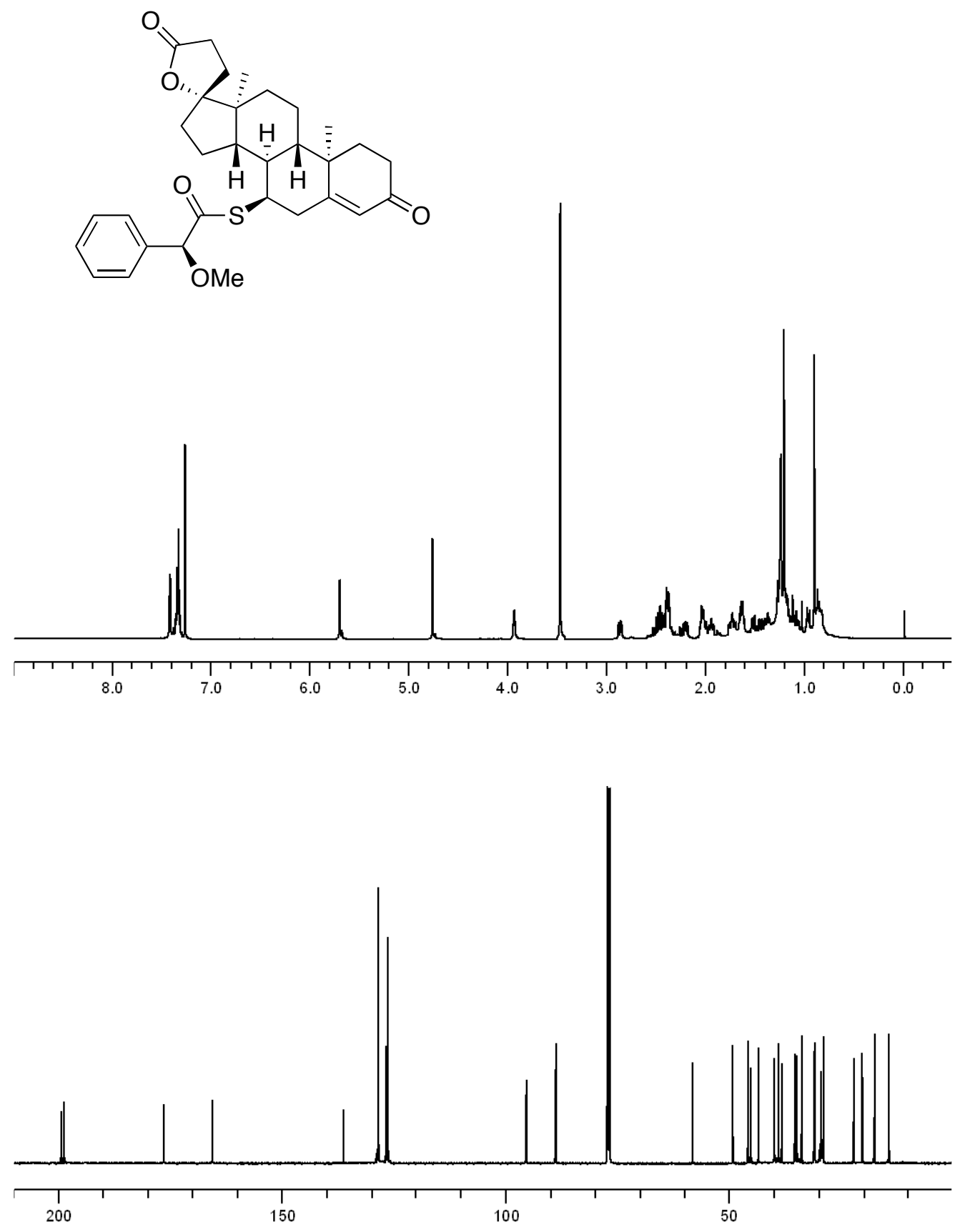

Figure 30S. ${ }^{1} \mathrm{H}$ NMR and ${ }^{13} \mathrm{C}$ NMR spectra of $(S)$-MPA-14. 

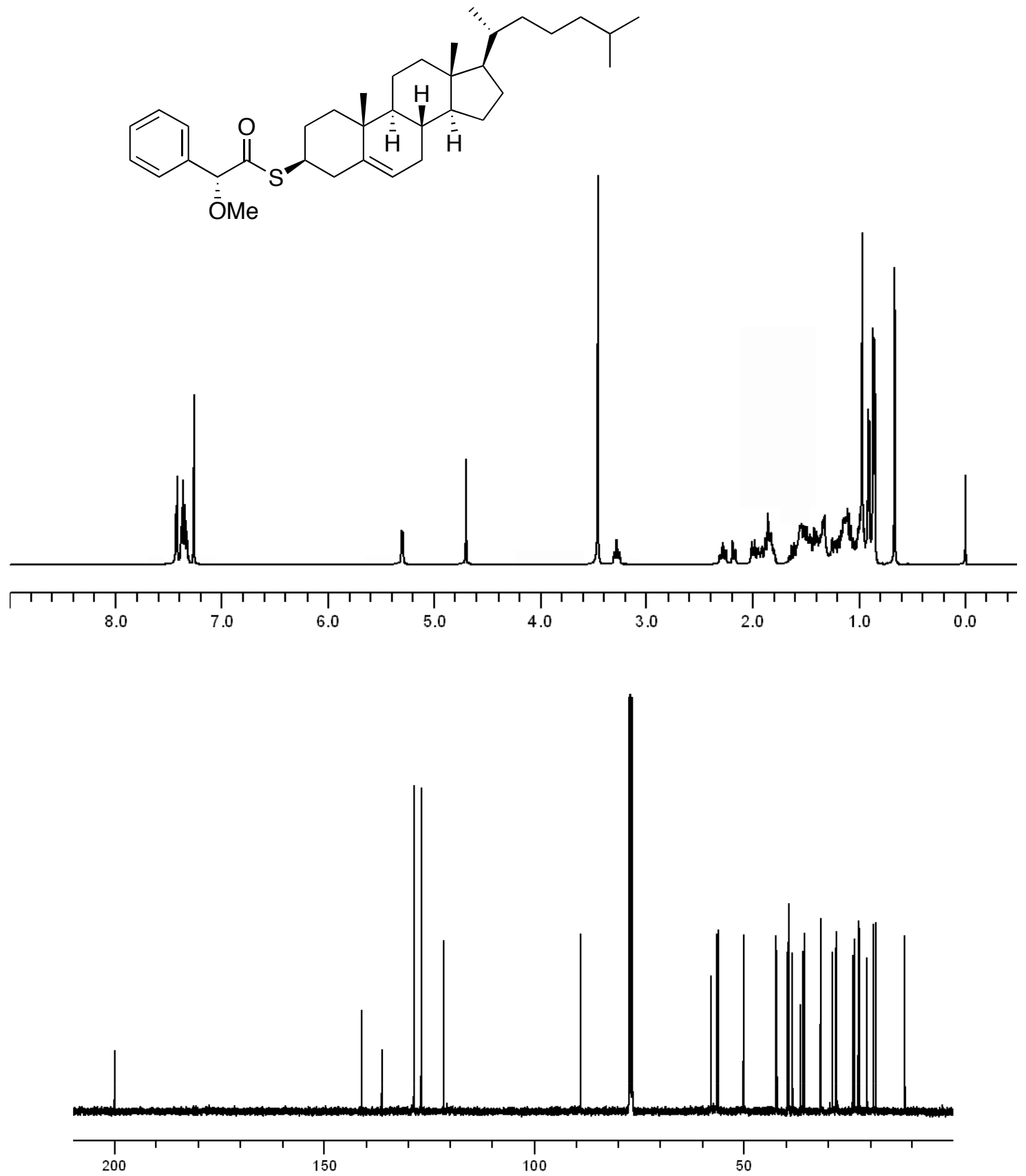

Figure 31S. ${ }^{1} \mathrm{H}$ NMR and ${ }^{13} \mathrm{C}$ NMR spectra of $(R)$-MPA-15. 

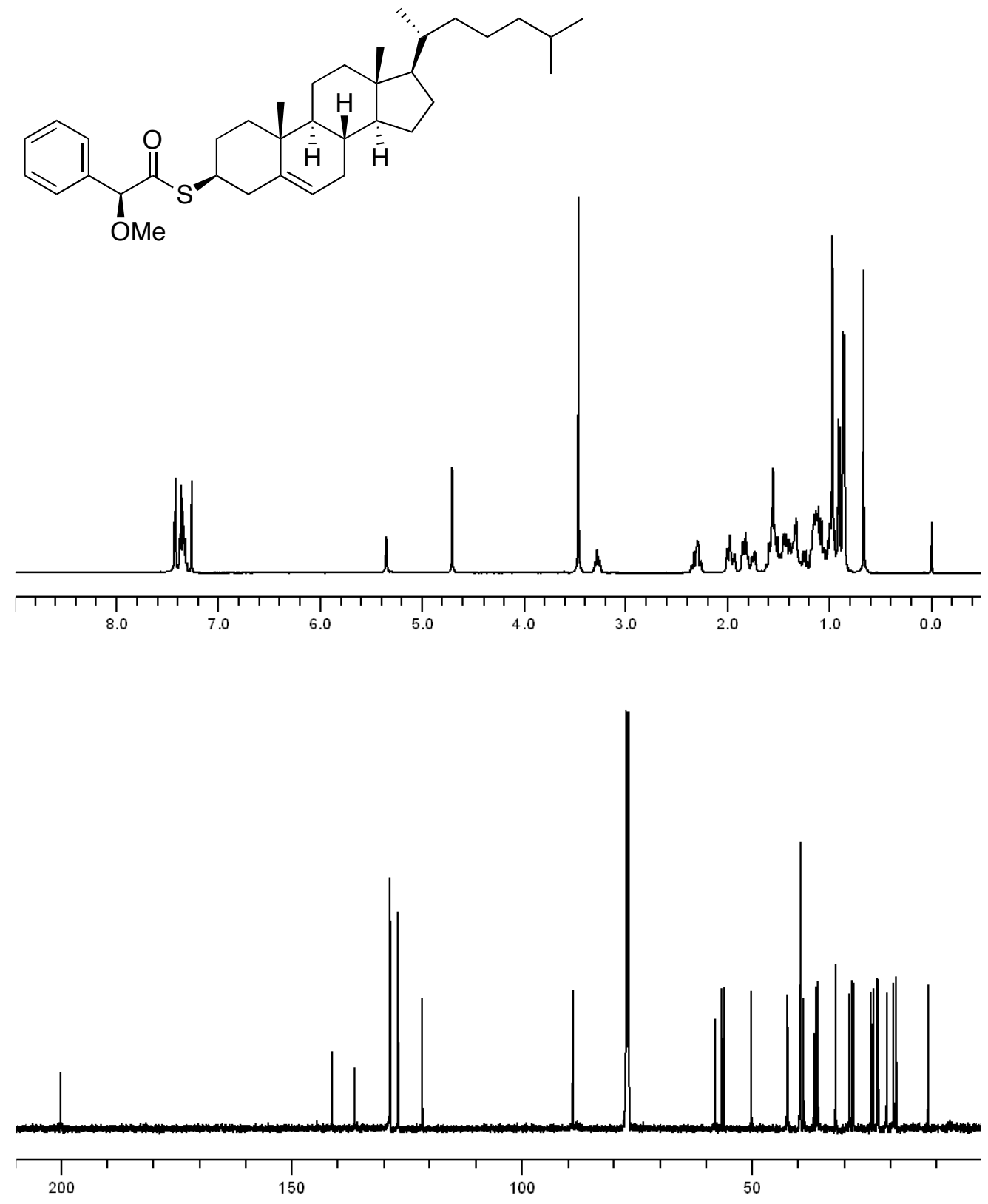

Figure 32S. ${ }^{1} \mathrm{H}$ NMR and ${ }^{13} \mathrm{C}$ NMR spectra of $(S)$-MPA-15. 

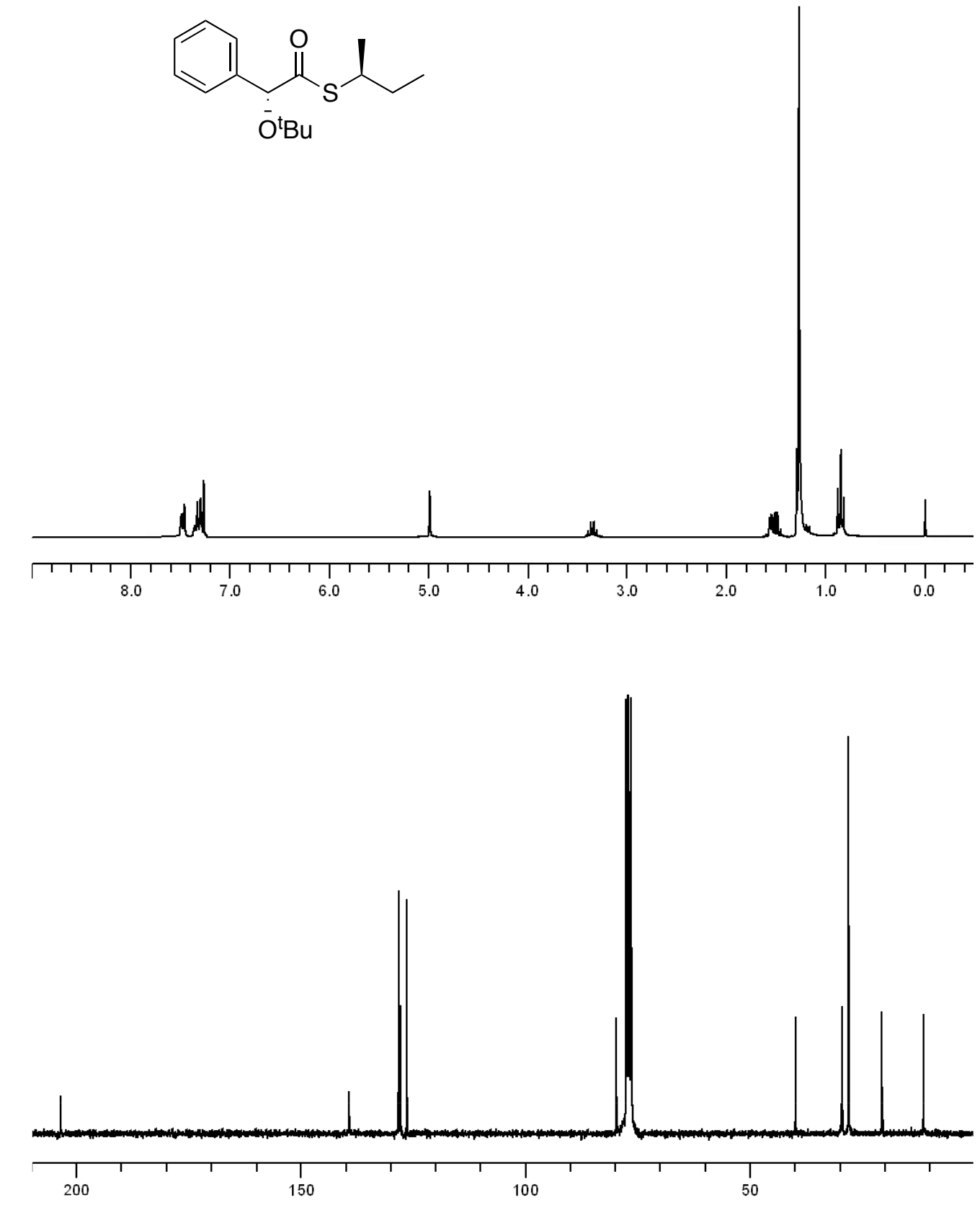

Figure 33S. ${ }^{1} \mathrm{H}$ NMR and ${ }^{13} \mathrm{C}$ NMR spectra of $(R)$-PTBA-5. 

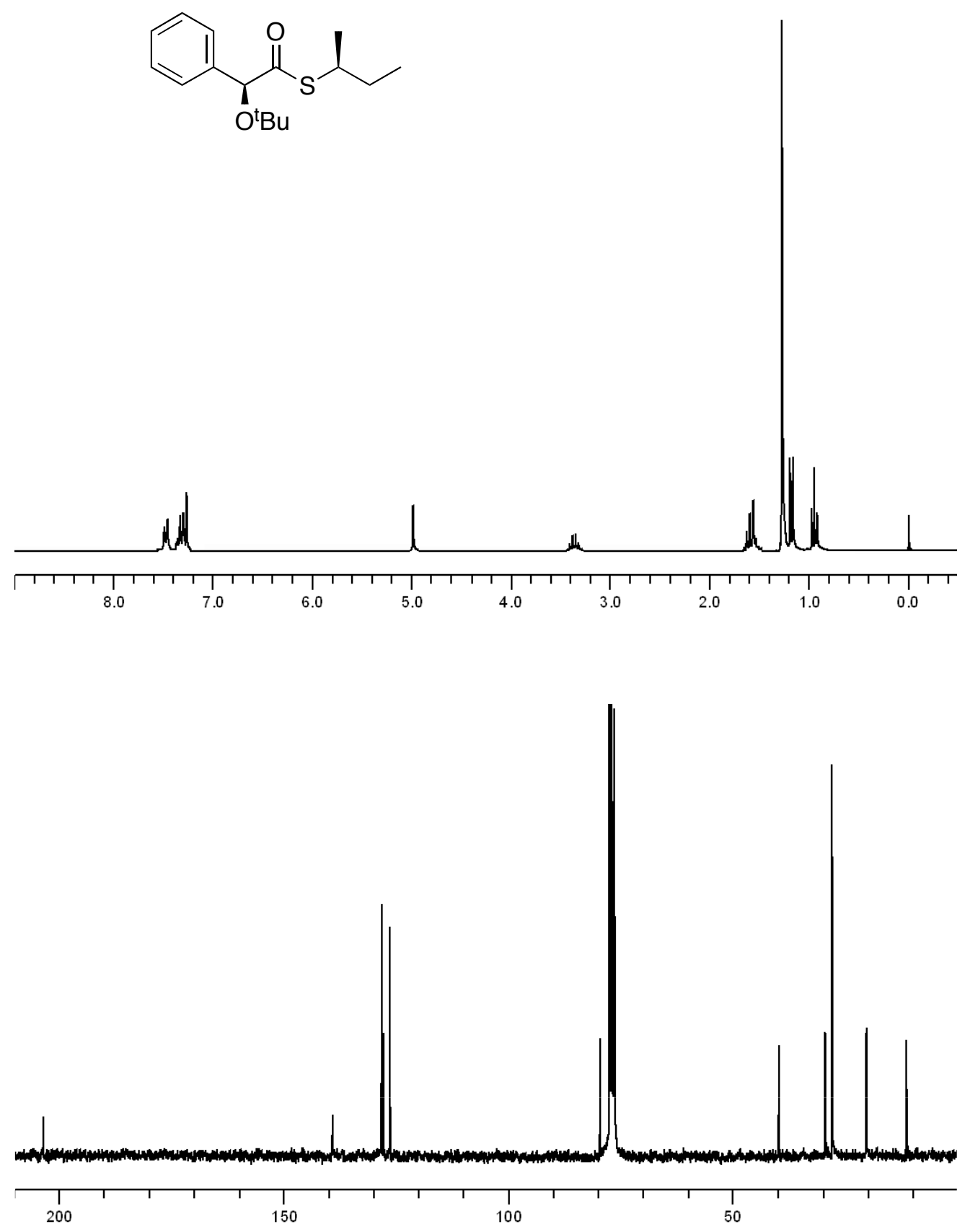

Figure 34S. ${ }^{1} \mathrm{H}$ NMR and ${ }^{13} \mathrm{C}$ NMR spectra of $(S)$-PTBA-5. 

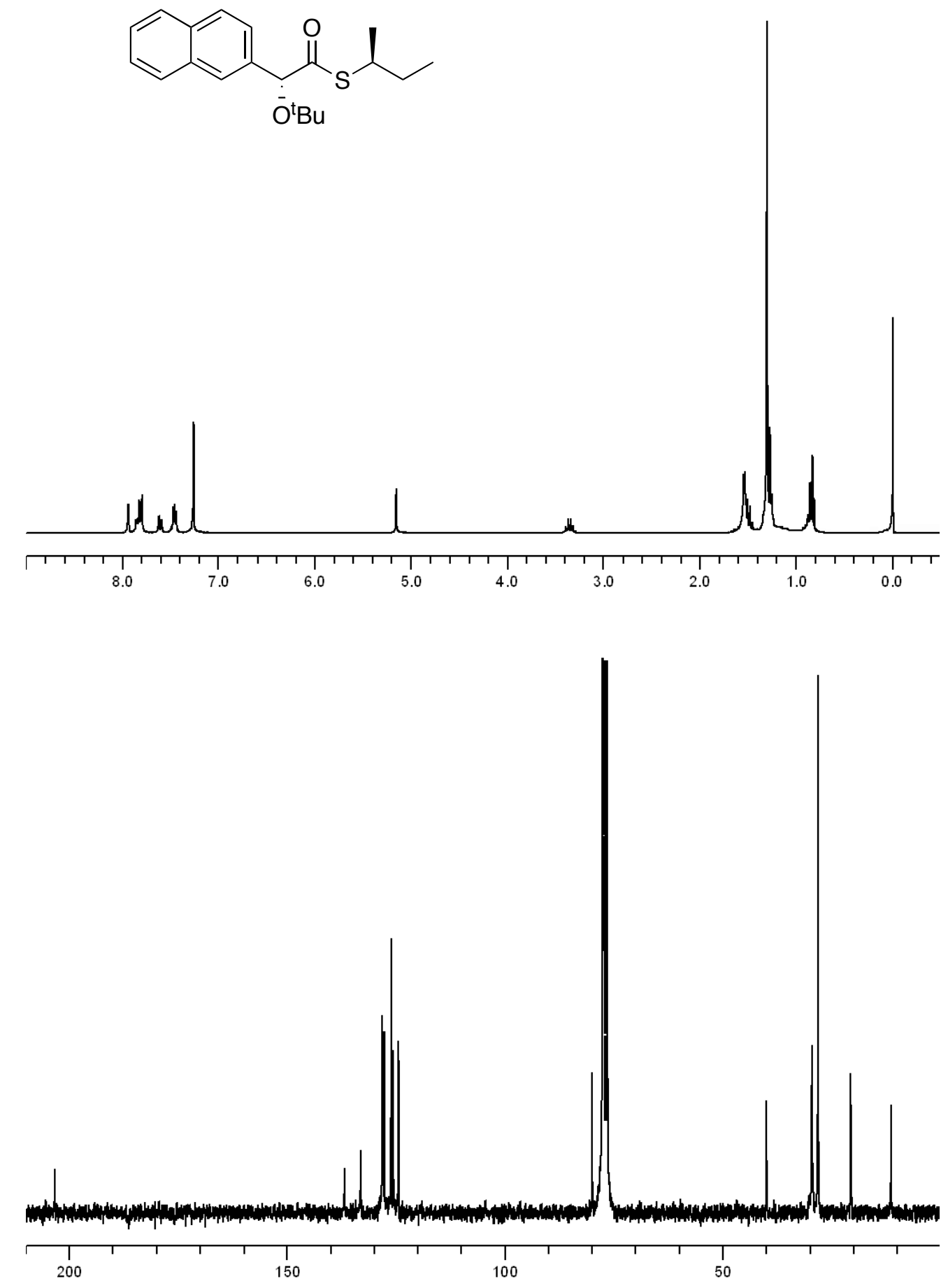

Figure 35S. ${ }^{1} \mathrm{H}$ NMR and ${ }^{13} \mathrm{C}$ NMR spectra of $(R)$-2-NTBA-5. 
<smiles>[GeH3]</smiles>
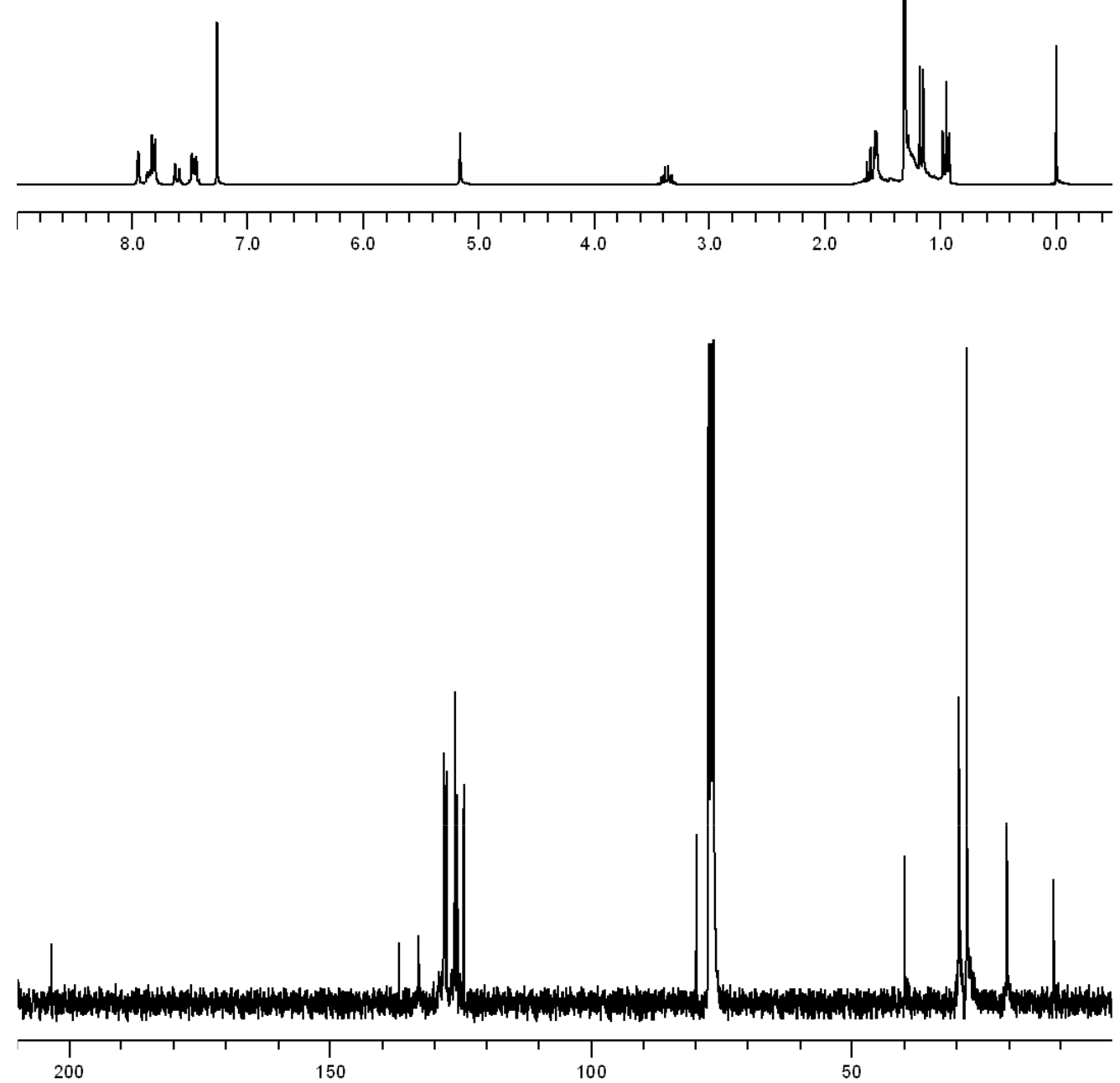

Figure 36S. ${ }^{1} \mathrm{H}$ NMR and ${ }^{13} \mathrm{C}$ NMR spectra of (S)-2-NTBA-5. 

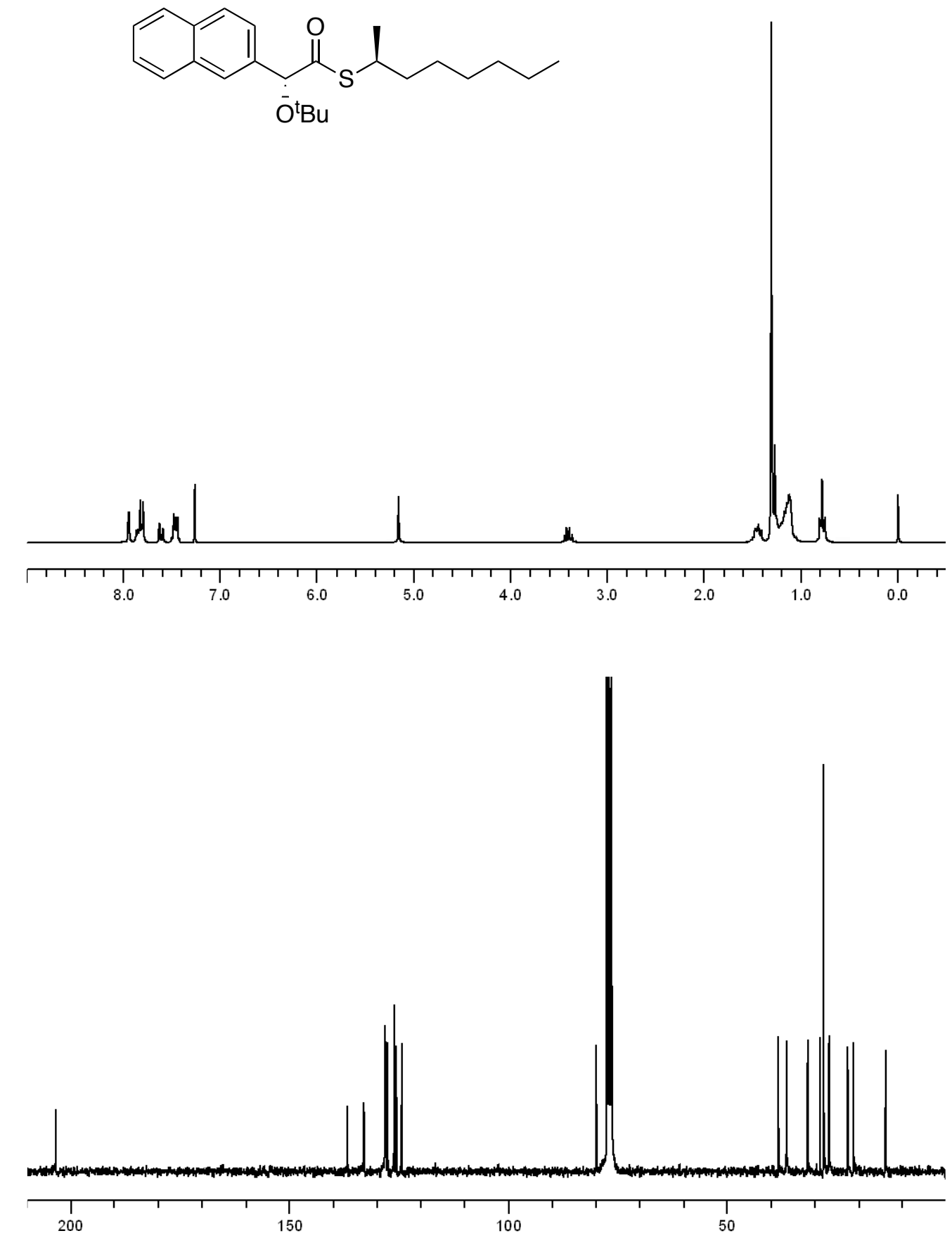

Figure 37S. ${ }^{1} \mathrm{H}$ NMR and ${ }^{13} \mathrm{C}$ NMR spectra of $(R)-2-N T B A-6$. 


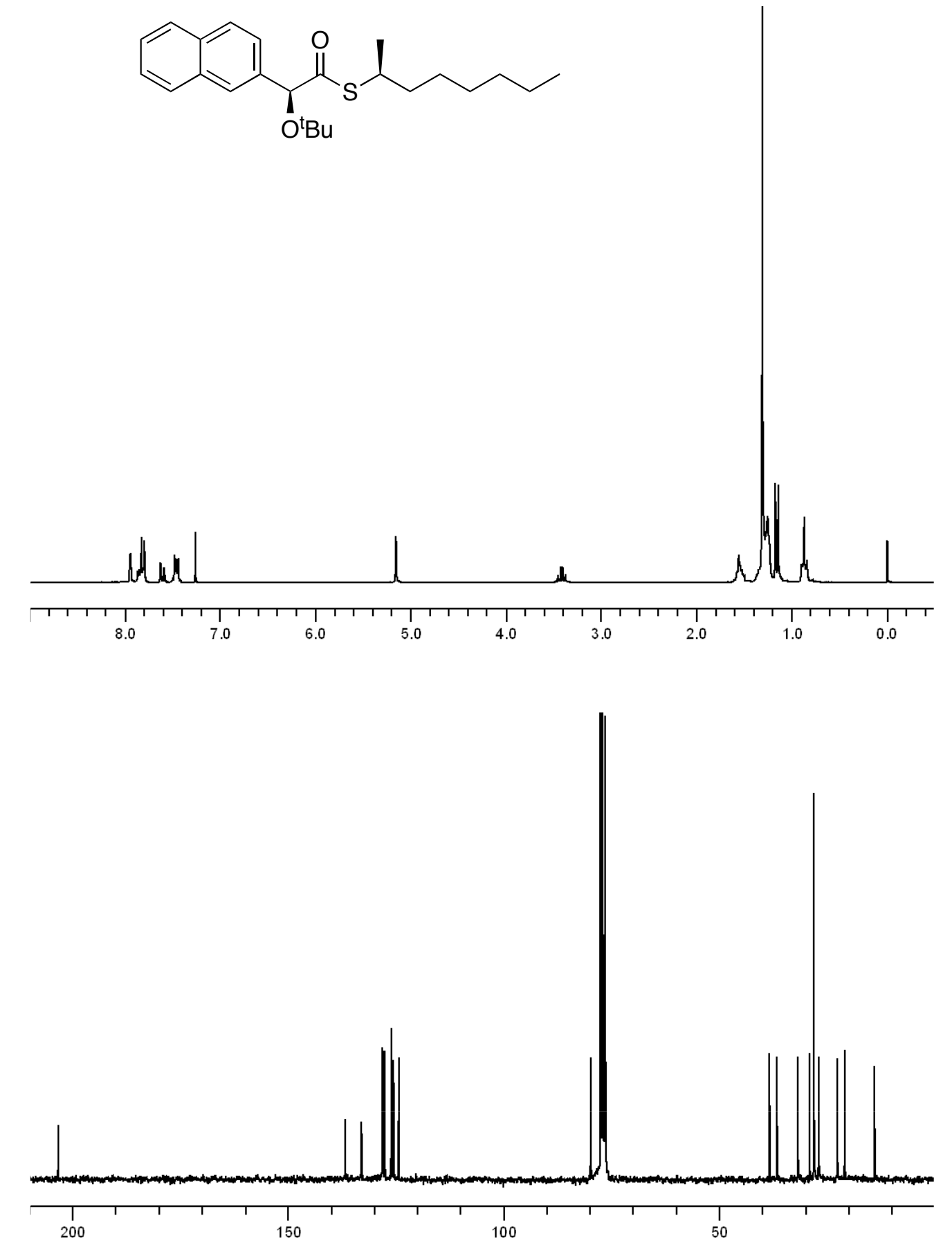

Figure 38S. ${ }^{1} \mathrm{H}$ NMR and ${ }^{13} \mathrm{C}$ NMR spectra of $(S)$-2-NTBA-6. 


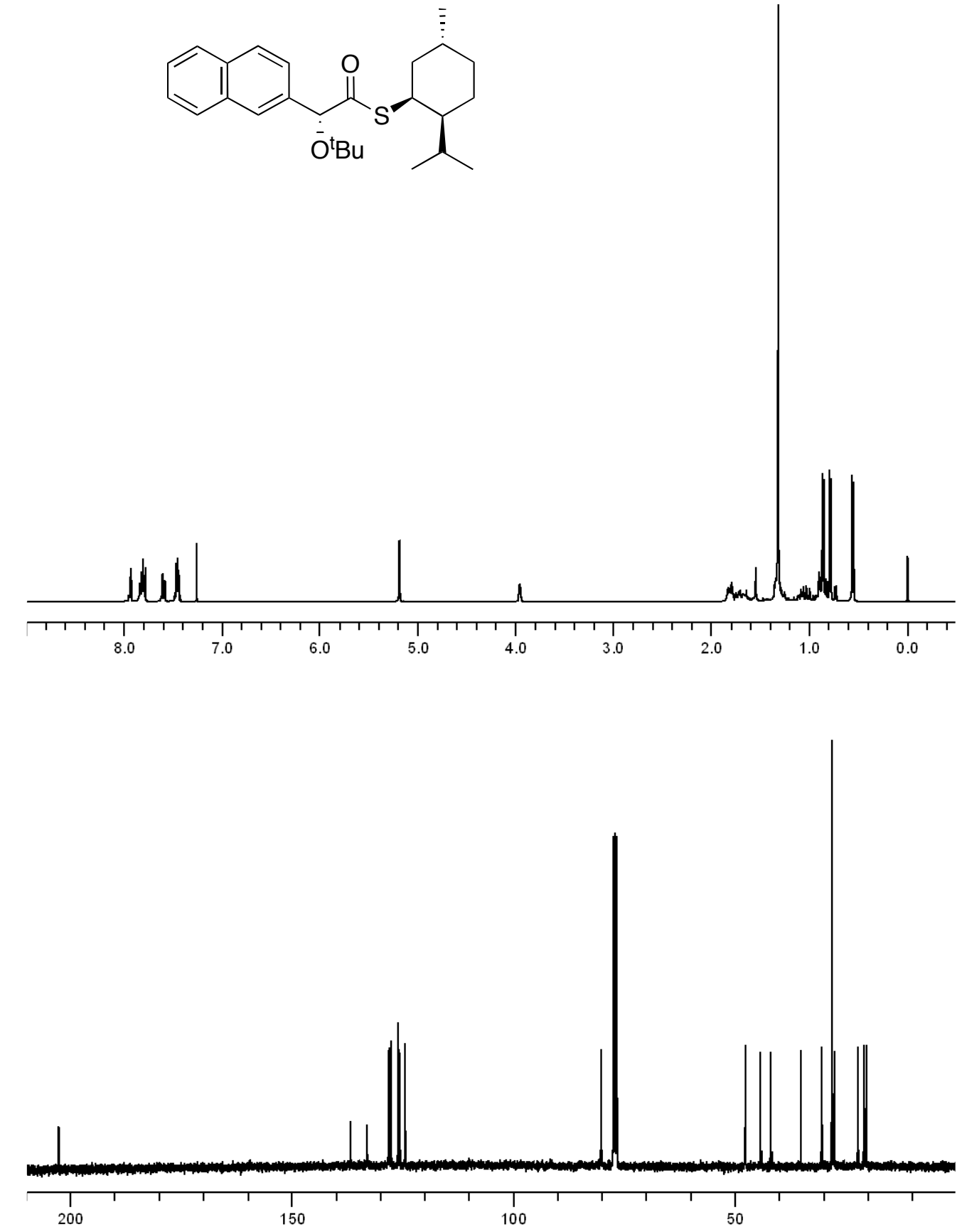

Figure 39S. ${ }^{1} \mathrm{H}$ NMR and ${ }^{13} \mathrm{C}$ NMR spectra of $(R)-2-N T B A-7$. 
<smiles>CCCC(O[C@H]1C[C@H](C)CCC1C(C)C)C(=O)[C@@H](OCC)c1ccc2ccccc2c1</smiles>
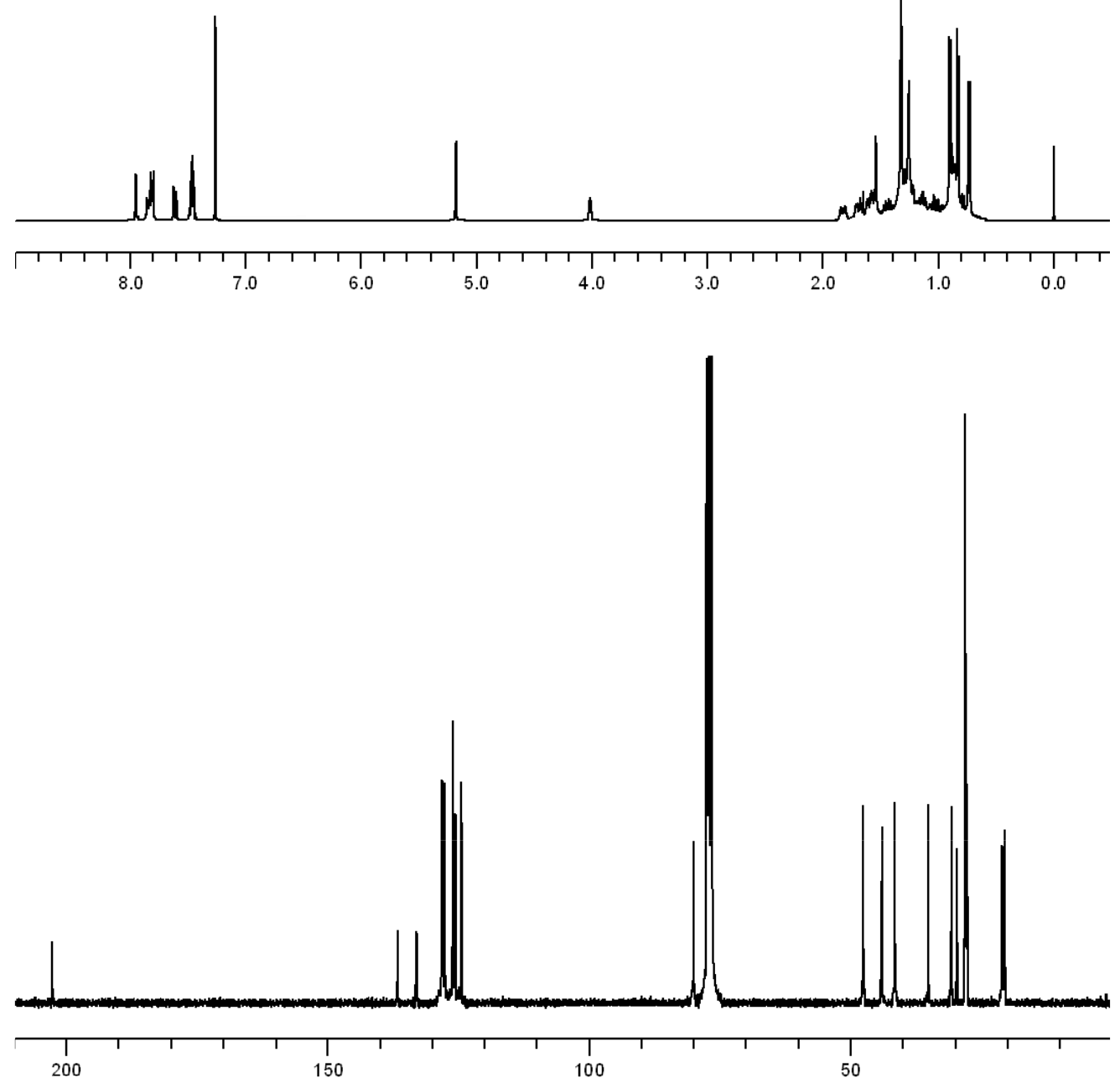

Figure 40S. ${ }^{1} \mathrm{H}$ NMR and ${ }^{13} \mathrm{C}$ NMR spectra of $(S)-2-N T B A-7$. 

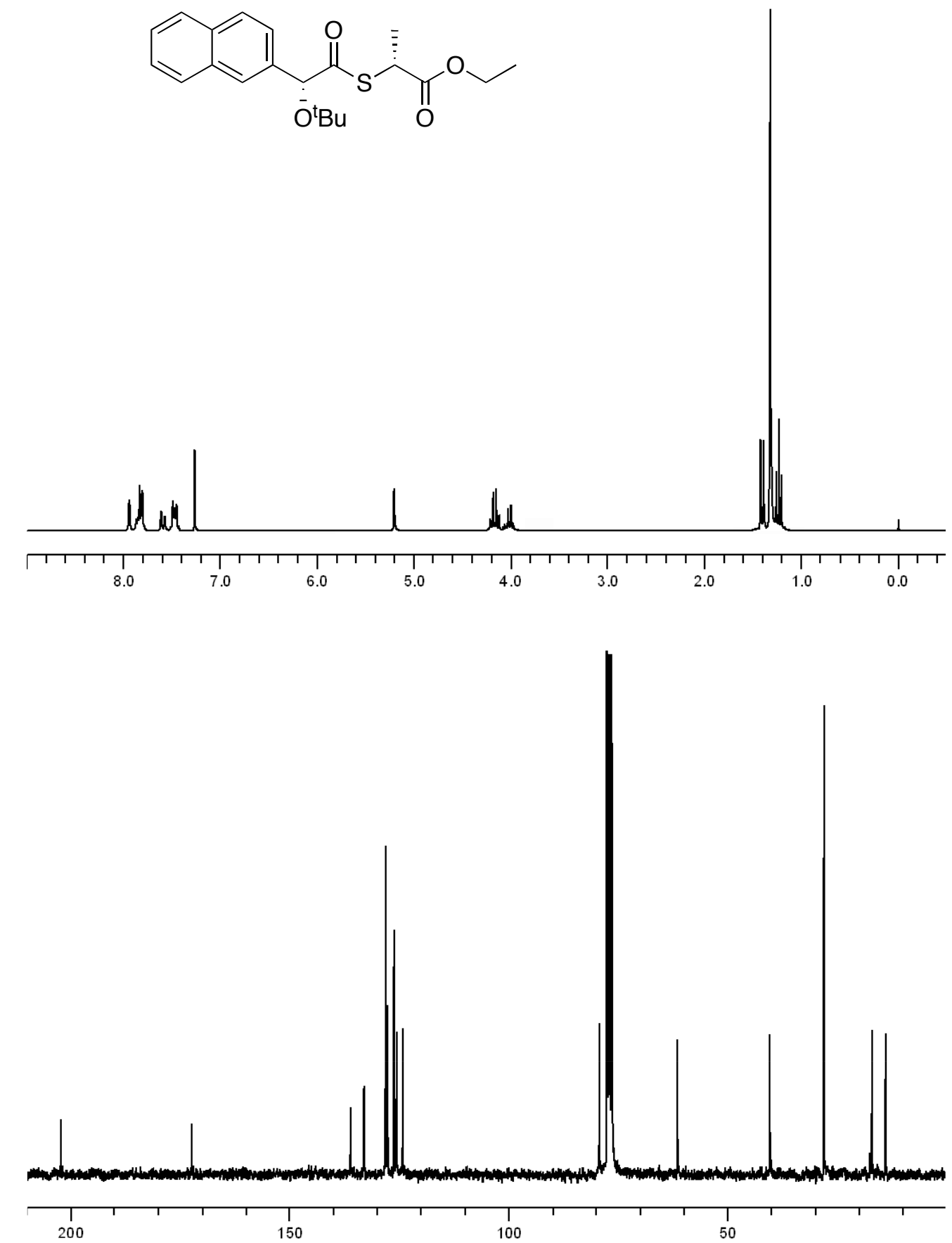

Figure 41S. ${ }^{1} \mathrm{H}$ NMR and ${ }^{13} \mathrm{C}$ NMR spectra of $(R)-2-\mathrm{NTBA}-8$. 
<smiles>CCOC(=O)[C@H](C)SC(=O)[C@H](O[GaH])c1ccc2ccccc2c1</smiles>
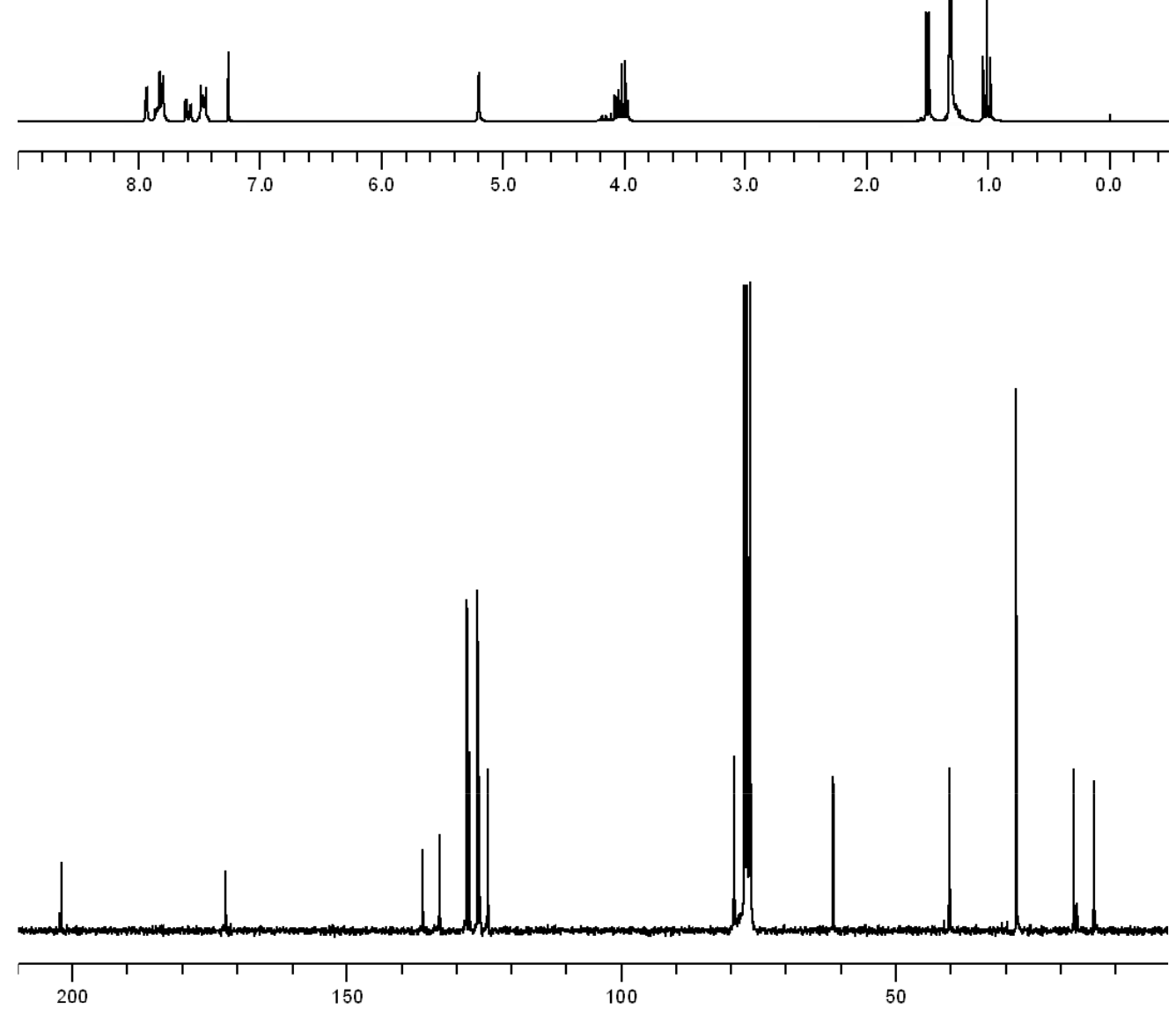

Figure 42S. ${ }^{1} \mathrm{H}$ NMR and ${ }^{13} \mathrm{C}$ NMR spectra of $(S)$-2-NTBA-8. 

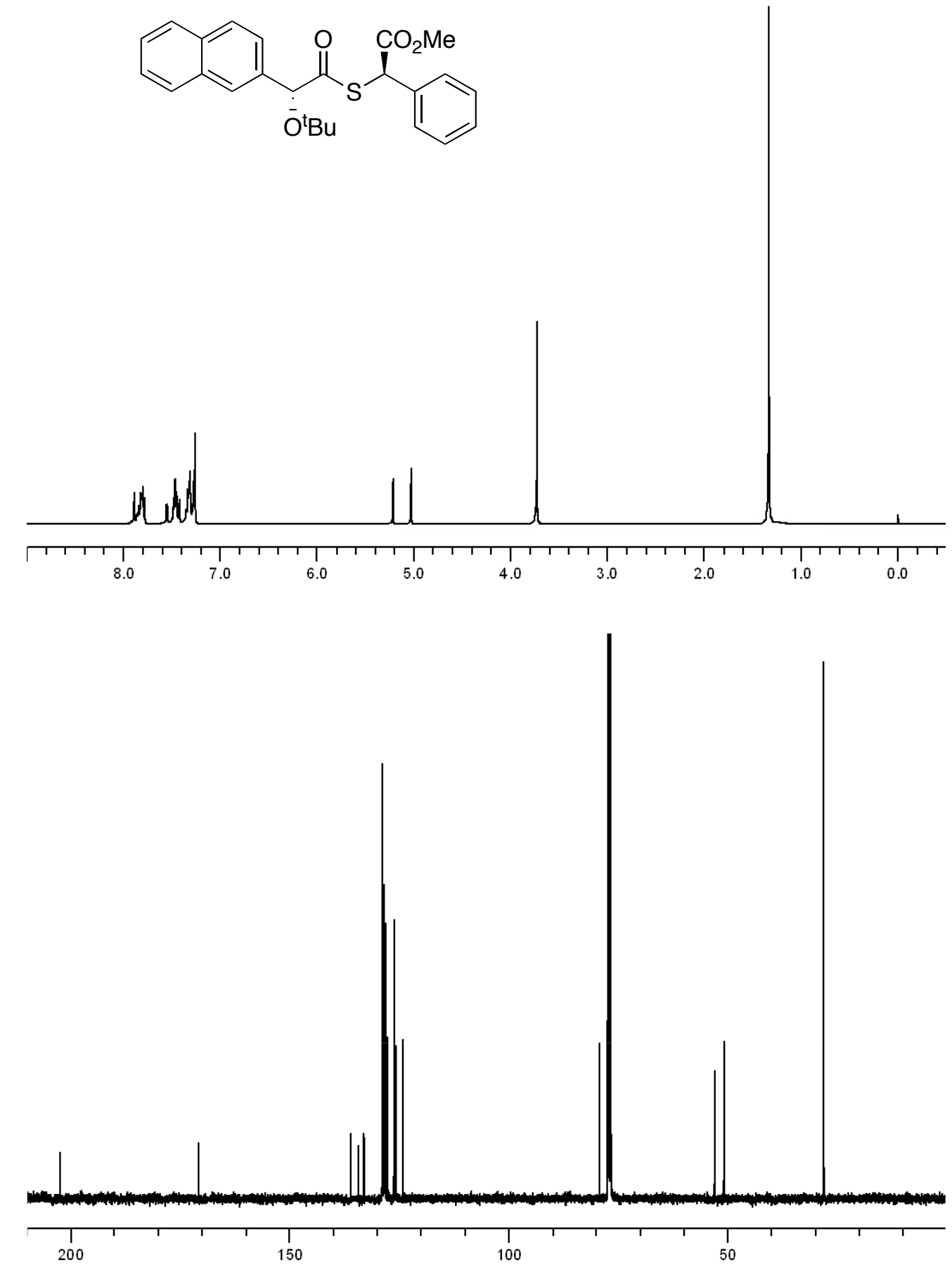

Figure 43S. ${ }^{1} \mathrm{H}$ NMR and ${ }^{13} \mathrm{C}$ NMR spectra of $(R)-2-N T B A-9$. 

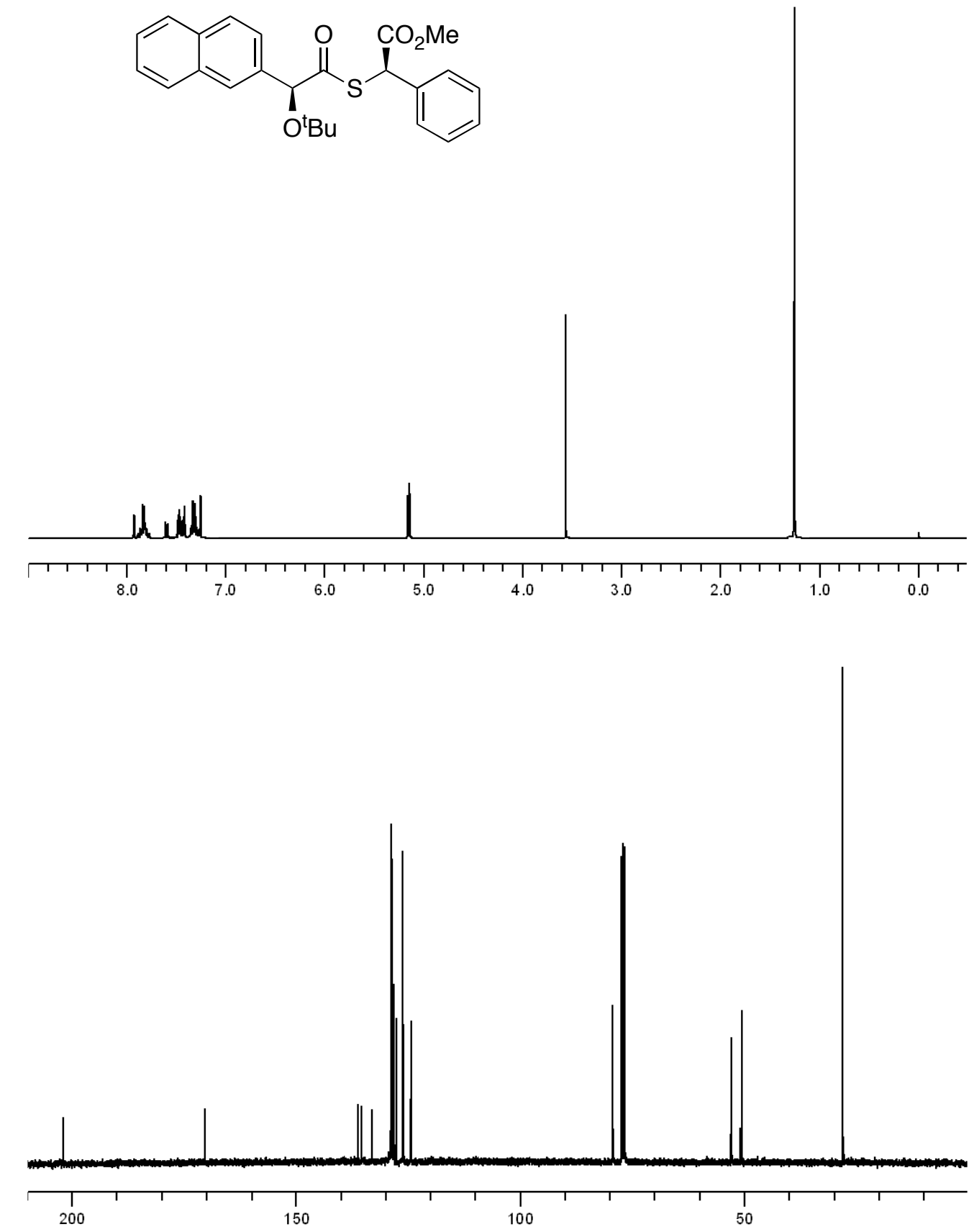

Figure 44S. ${ }^{1} \mathrm{H}$ NMR and ${ }^{13} \mathrm{C}$ NMR spectra of (S)-2-NTBA-9. 

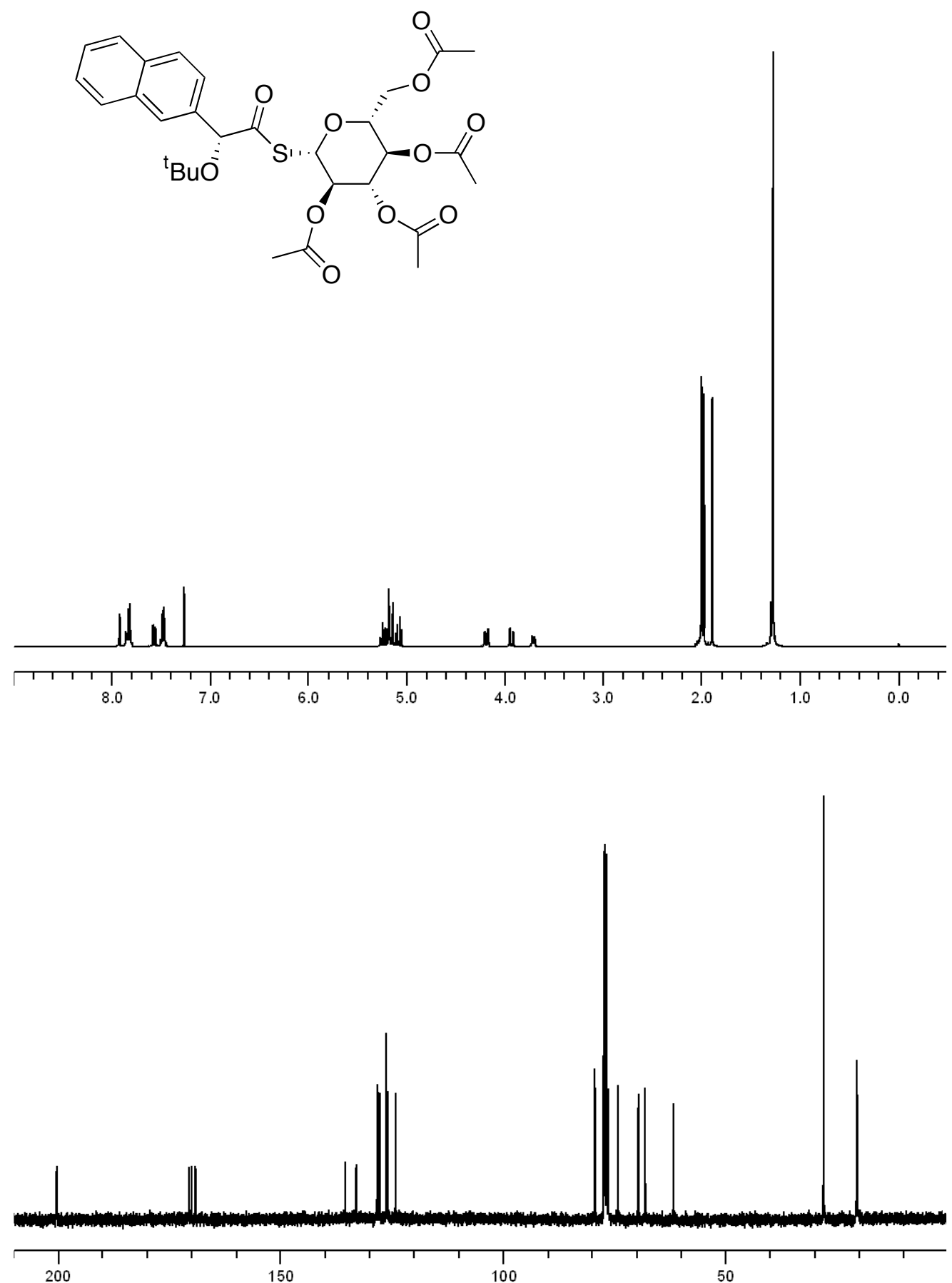

Figure 45S. ${ }^{1} \mathrm{H}$ NMR and ${ }^{13} \mathrm{C}$ NMR spectra of $(R)-2-\mathrm{NTBA}-10$. 

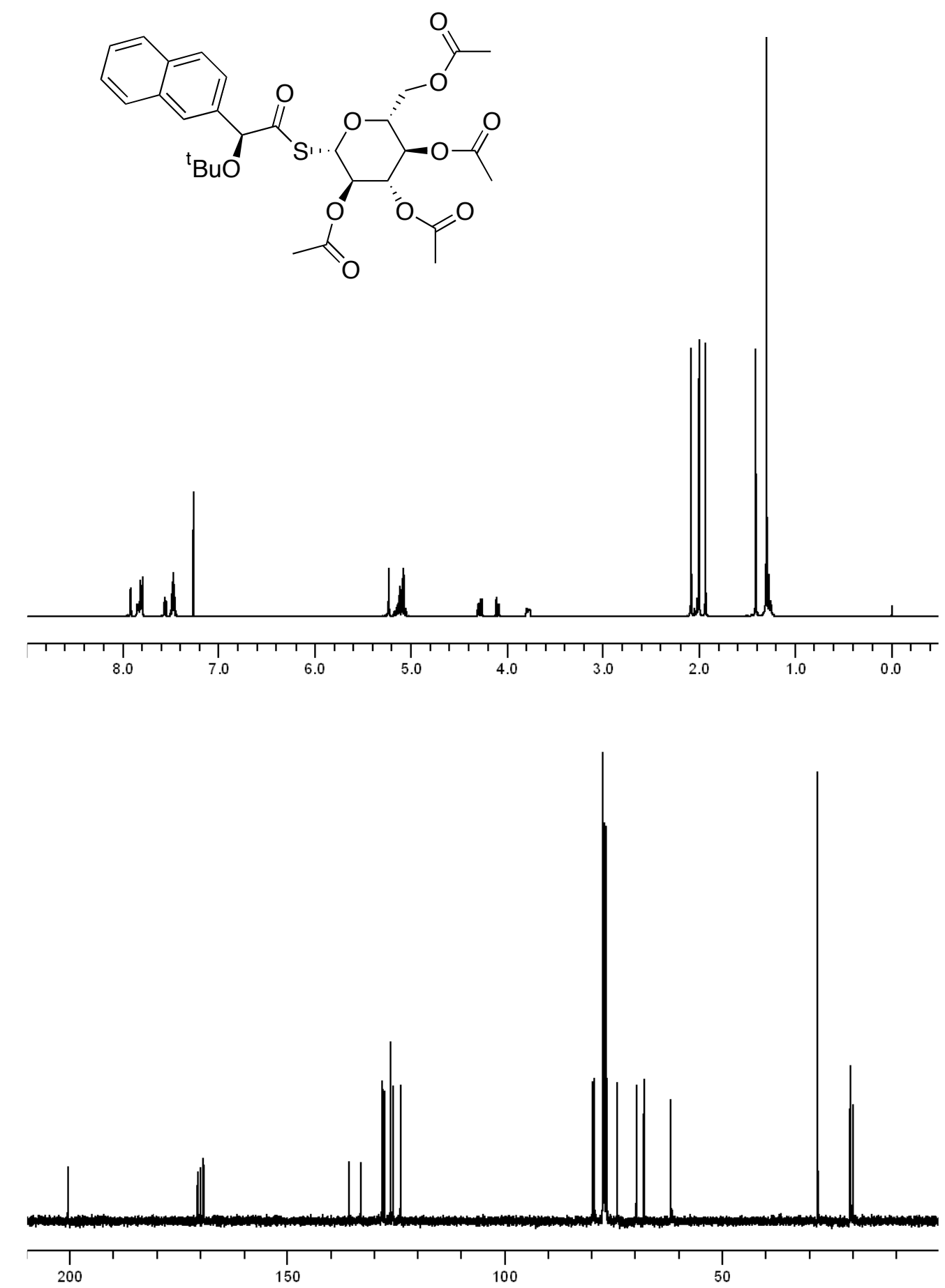

Figure 46S. ${ }^{1} \mathrm{H}$ NMR and ${ }^{13} \mathrm{C}$ NMR spectra of $(S)$-2-NTBA-10. 

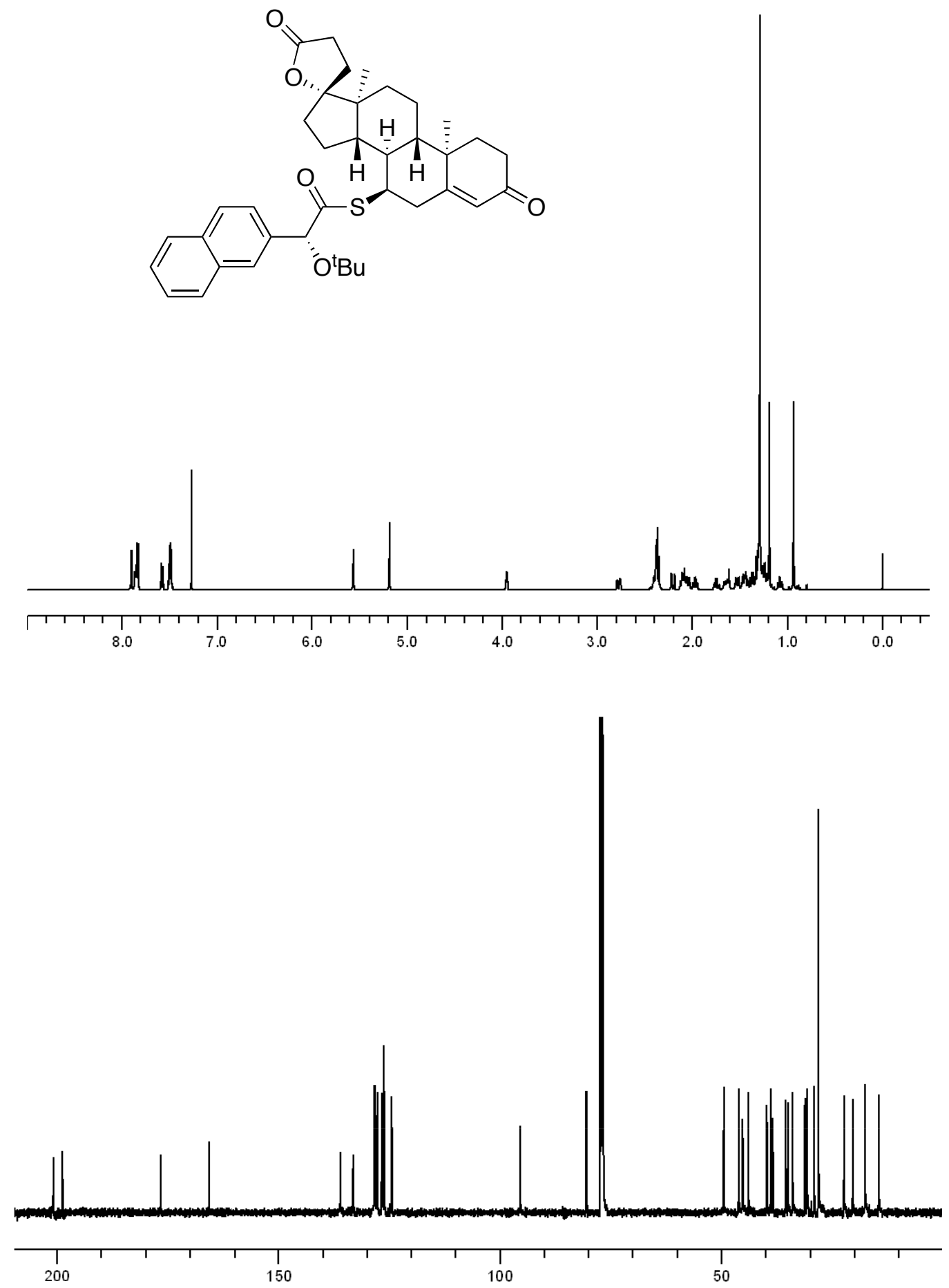

Figure 47S. ${ }^{1} \mathrm{H}$ NMR and ${ }^{13} \mathrm{C}$ NMR spectra of $(R)-2-\mathrm{NTBA}-14$. 

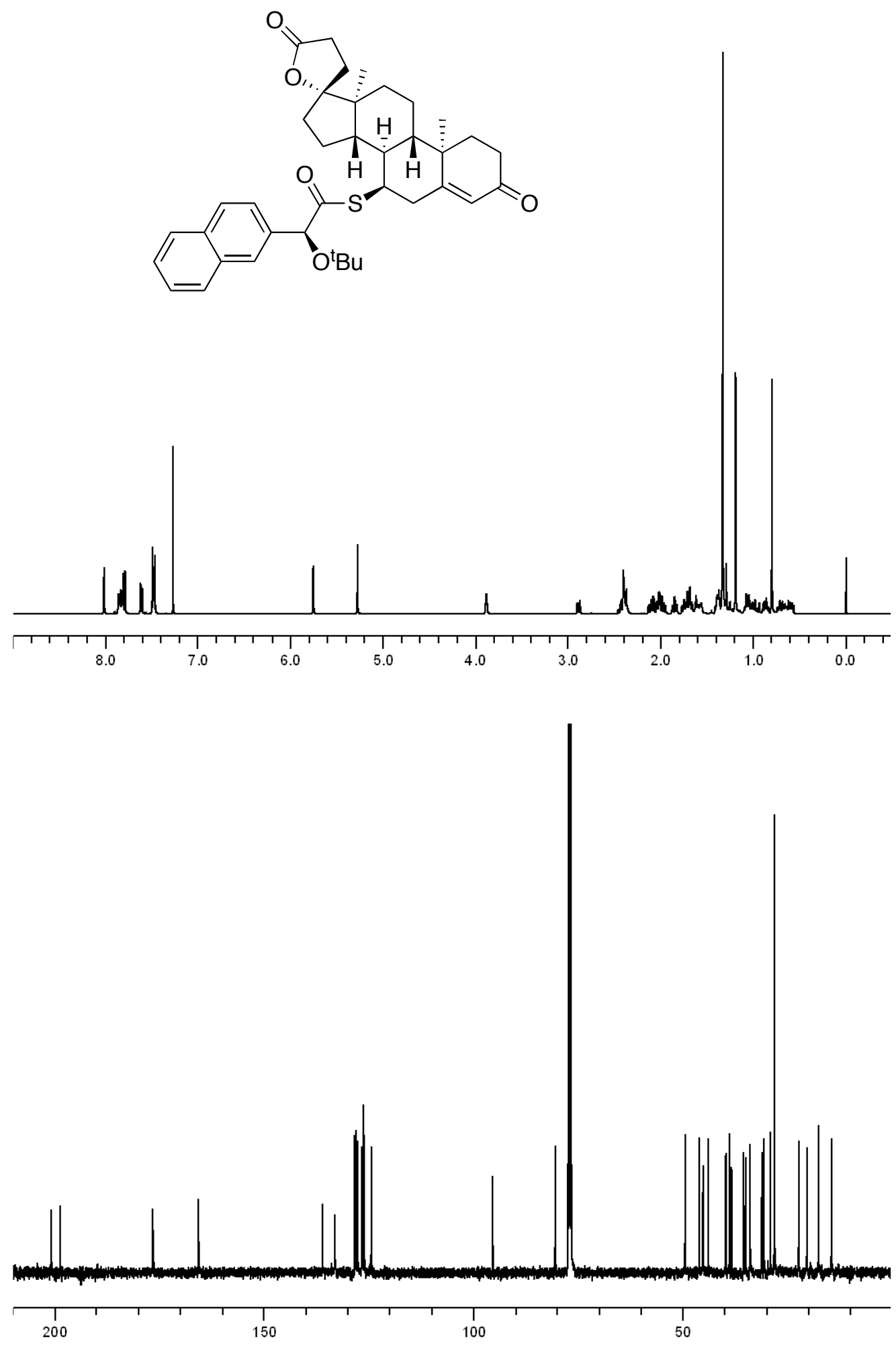

Figure 48S. ${ }^{1} \mathrm{H}$ NMR and ${ }^{13} \mathrm{C}$ NMR spectra of $(S)-2-N T B A-14$. 

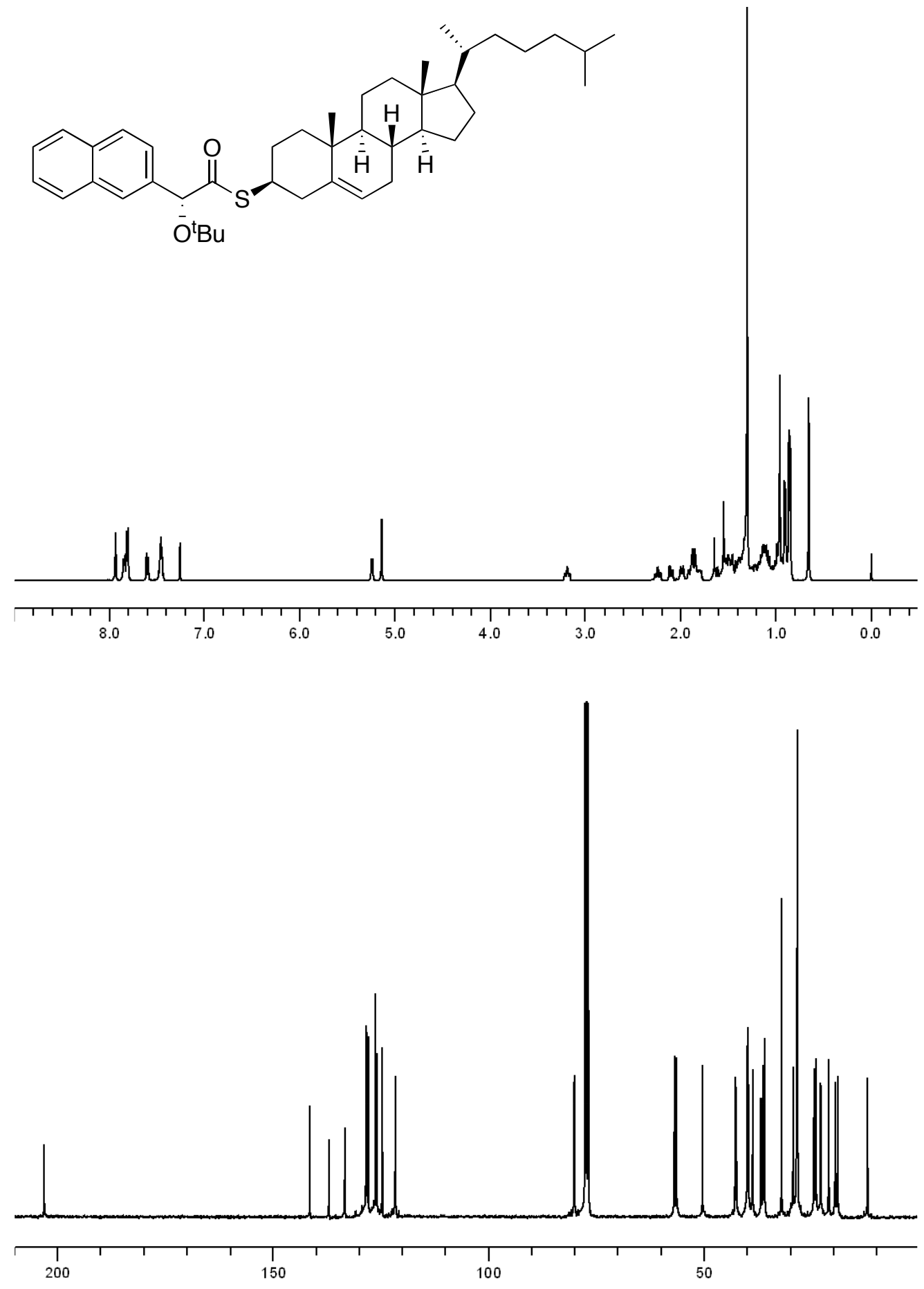

Figure 49S. ${ }^{1} \mathrm{H}$ NMR and ${ }^{13} \mathrm{C}$ NMR spectra of (R)-2-NTBA-15. 

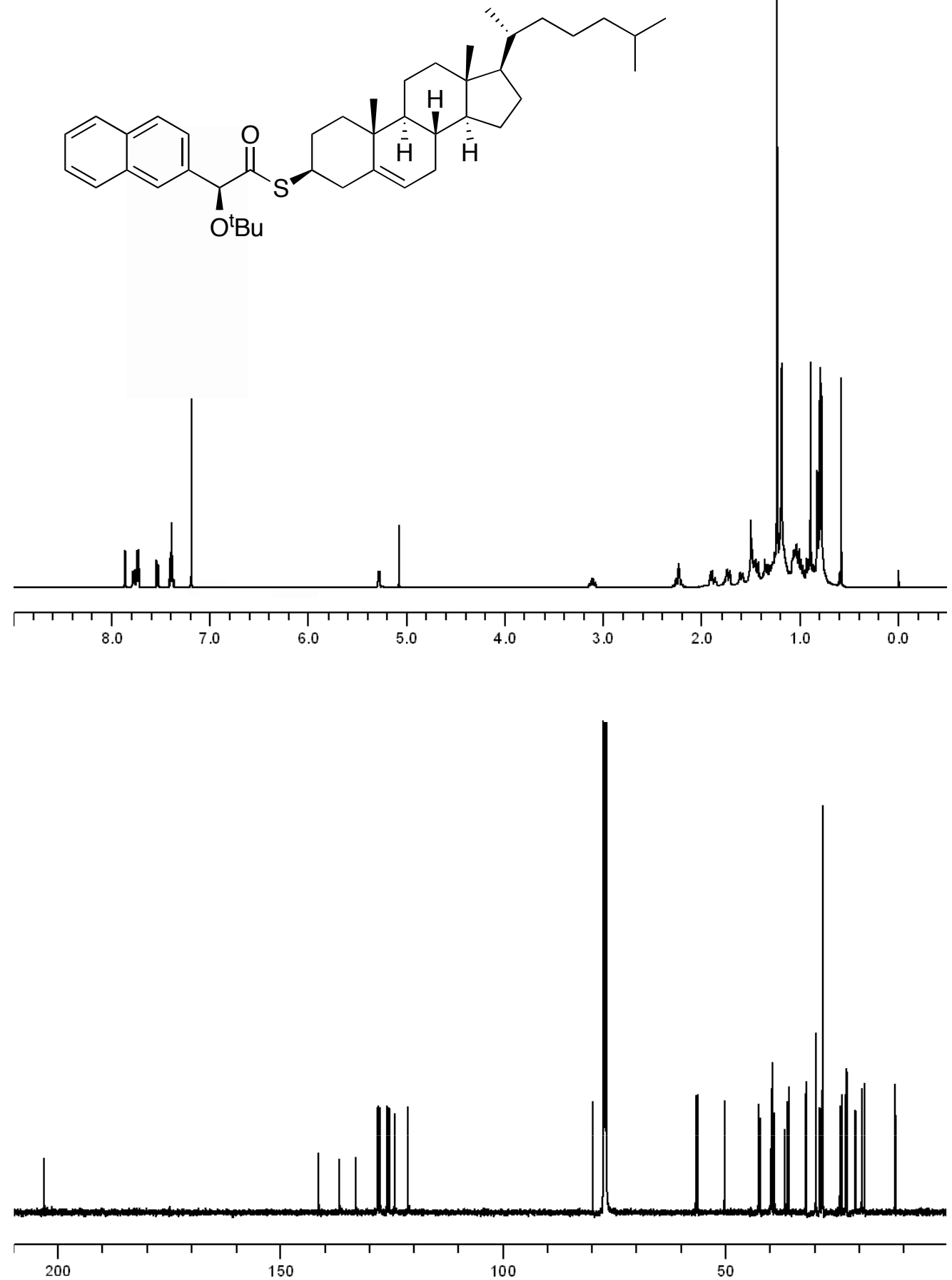

Figure 50S. ${ }^{1} \mathrm{H}$ NMR and ${ }^{13} \mathrm{C}$ NMR spectra of $(S)-2-\mathrm{NTBA}-15$. 
Crystal Structure of $(S)$-MPA thioester of 1-thio- $\beta$-D-glucose tetraacetate $[(S)$ MPA-10].

Crystal data. $\mathrm{C}_{23} \mathrm{H}_{28} \mathrm{O}_{11} \mathrm{~S}, M=512.51$, monoclinic, $a=27.301(8) \AA, b=9.969(3) \AA ⿻$ $=18.683(5) \AA, V=4924(2) \AA^{3}, T=100.0(1) \mathrm{K}$, space group $C 2, Z=8,22902$ reflections measured, 10233 unique $\left(R_{\text {int }}=0.0545\right)$ which were used in all calculations. The final $w R\left(F_{2}\right)$ was 0.1342 (all data). CCDC 650882 contains the supplementary crystallographic data for this paper. These data can be obtained free of charge from The Cambridge Crystallographic Data Centre via www.ccdc.cam.ac.uk/data_request/cif

Figure 51S. Diffraction X-ray spectroscopy of the $(S)$-MPA thioester of 1-thio- $\beta$-Dglucose tetraacetate (10).

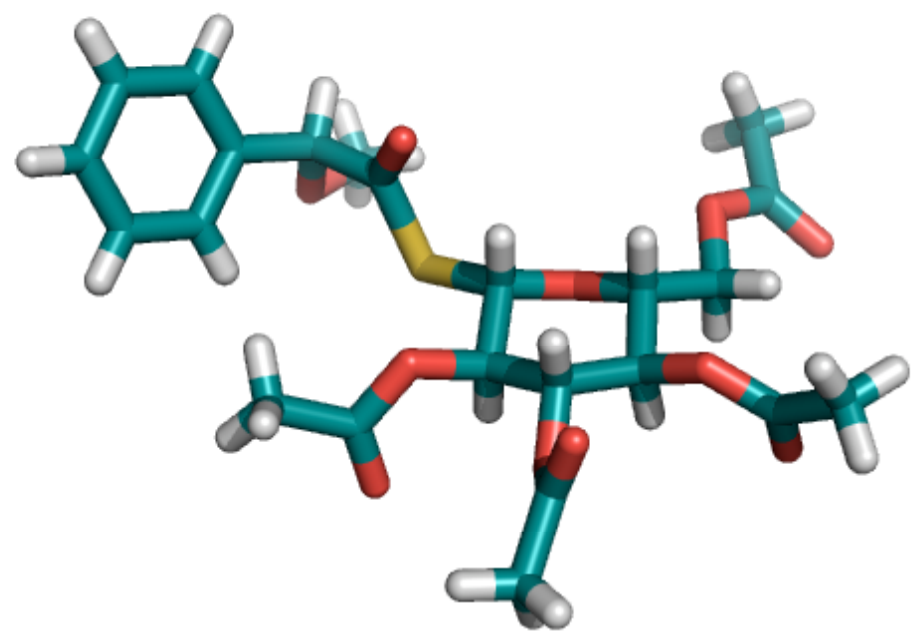

